\title{
Hans-Otto Walther \\ Hyberbolic periodic solutions, heteroclinic connections and transversal homoclinic points in autonomous differential delay equations
}


Preliminaries

Chapter I: Hyperbolic periodic solutions

1. Periodic solutions

2. Linearization

3. Translation by variational equations along periodic solutions

4. Eigenvalues as zeros of analytic functions

5. zeros of $q_{a}$

6. Hyperbolicity

7. Comparison results

Chapter II: On hyperbolic fixed points

1. Local invariant manifolds

2. Proof of Proposition 1.1

Chapter III: Poincare'maps and solutions close to $\mathrm{x}_{a}$

1. Poincare'maps

2. Departure of solutions from the local unstable manifold of $P$

3. Intersecting $\mathrm{H}$, above and below the local stable manifold

4. A condition for transversality

Chapter IV: Heteroclinic connections between periodic orbits, homoclinic points of poincare-maps, transversality

1. Escape from $x$ in domains of monotonicity

2. Periodic nonlinearities

3. Transport of $x \in U$ and $v \in T, U$ for $0 \leqq t \leqq t_{+}$

4. Heteroclinic solutions and transversal homoclinic points

Chapter V: on chaotic behavior

References 
Abstract: We study autonomous differential delay equations

$$
\dot{x}(t)=g(x(t-1))
$$

for $C^{1}$-nonlinearities $g: \underline{R} \rightarrow \underline{R}$ which are periodic in $x$ and sine-like, i.e., with $g(0)=0$ and with one (simple) zero in the period interval

$(0, \omega)$. Such equations model delayed feedback on the circle.

It is proved that within this class there exist nonlinearities with. - hyperbolic periodic solutions close to the equilibria $j \omega, j \in \underline{Z}$, and with

- heteroclinic solutions connecting the periodic orbit close to jw to the periodic orbit close to $(j+1) \omega$.

In addition, a transversality property is established.

The corresponding Poincare'map, which has no continuous inverse, constitutes a discrete (semi-) dynamical system in an infinite dimensional phase space with a transversal homoclinic point. A result of Hale and Lin is applied to describe in terms of symbolic dynamics the chaotic motion close to the homoclinic loop of the map, or equivalently, close to the heteroclinic connections of the continuous semiflow.

Key words and phrases: Differential delay equation, autonomous, infinite dimensional system, hyperbolic, periodic orbit, heteroclinic, noninvertible Poincare'map, transversal homoclinic point, symbolic dynamics

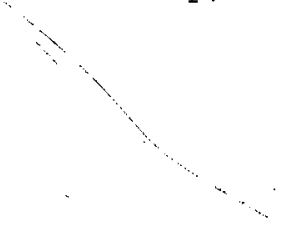

INTRODUCTION

Autonomous diffexential-delay equations

(g) $\quad \dot{x}(t)=g(x(t-1))$

with $g: R \rightarrow \underline{R}$ periodic model delayed feedback for a state variable on the circle.

If $\gamma_{z}$ is a zero of $g$ with

$\left(\xi-\gamma_{z}\right) \cdot g\left(\xi-\gamma_{z}\right)<0$ for $\xi-\gamma_{z} \neq 0$ small

then feedback is negative with respect to the rest point given by $\gamma_{z}$. Continuity of $g$ implies a zero in the interval $\left(\gamma_{z}-\omega, \gamma_{z}\right)$ where $\omega$ denotes the minimal period of $g$. In the simplest case there is only one zero in $\left(\gamma_{z}-\omega, \gamma_{z}\right)$; we may assume that $g$ is sine-like in the sense $g(0)=0,0<g$ in $\left(0, \gamma_{z}\right), g<0$ in $\left(\gamma_{z}, \omega\right)$.

Such equations occur in a number of applications. We only mention models for phase-locked loops $[2,5,30]$.

Initial values $\phi \in C=C([-1,0], \underline{R})$ determine solutions $x^{\phi}:[-1, \infty) \rightarrow \underline{R}$. Setting

$$
x(t, \phi):=x_{t}^{\phi}:=x^{\phi}(t+\cdot) \mid[-1,0] \text { for } t \geqq 0, \phi \in C,
$$

one obtains a continuous semiflow $\mathrm{X}: \mathrm{R}^{+} \times \mathrm{C} \rightarrow \mathrm{C} \cdot \mathrm{X}$ does not extend to a flow; there is no underlying vectorfield, no matter how smooth $g$ is.

The dynamics of these semiflows is very rich. Depending on further properties of $g$, one finds various kinds of periodic solutions, heteroand homoclinic solutions, and in parameterized equations, local and nonlocal bifurcations $[5,31,33,34,35]$.

Chaotic motion has been established for special aifferential delay equations with a somewhat different feedback structure $[32,23,10,11,9]$, The nonlinearities in $[32,10,11,9]$ are smoothed step functions, especially designea so that periodic and homoclinic solutions are found by explicit computation. Initially different solutions flow together in finite time and enter 2-dimensional subsets of $\mathrm{C}$; one obtains Poincare'maps on onedimensional domains, and chaos can be derived from results on interval Received by the editors May 23, 1988 and, in revised form october 10, 1988 
maps $[16,15,24]$

The present paper contains a more general approach to chaotic differential delay equations. It does not rely on nonlinearities which are smoothed step functions. Geometric arguments replace explicit calculations. The structure finally implying chaos is more complicated than in the former examples. A reduction to interval maps is no longer at hand.

Before giving results, let us recall the work of Smale from $[26,27]$ : Consider a diffeomorphism $f$ in a finite-dimensional space with hyperbolic fixed point $x^{*}$, i.e., there are no eigenvalues of $D f\left(x^{*}\right)$ on the unit circle $\underline{s}$. If the stable and unstable (inmersed) manifolds of $x^{*}$ intersect transversally in a homoclinic trajectory $\left(x_{n}\right)_{-\infty}^{\infty}$, i.e.,

$f\left(x_{n-1}\right)=x_{n} \rightarrow x^{*}$ as $|n| \rightarrow \infty$, then there is chaos: On an invariant subset, an iterate of $f$ is topologically conjugate to a shift on a space of symbol sequences [6]. See also $[17,19,21,22]$.

Silnikov, who studied a hyperbolic periodic orbit of a vectorfield with transversal stable and unstable manifolds, constructed a neighborhood so that all trajectories in this neighborhood are represented by symbol sequences [25].

A generalization of Śilnikov's result to infinite dimension, for maps which are not necessarily injective, is due to Hale and Lin (Theorem 5.2 [8]). A new proof of Hale's and Lin's result, now based on hyperbolic structures for arbitrary $\mathrm{C}^{1}$-maps and on a generalized shadowing lemma [28], has been given in [29].

In the present paper we prove existence of sine-like nonlinearities $g$ with hyperbolic periodic solutions

$$
\ldots, x-\omega, x, x+\omega, \ldots
$$

and heteroclinic solutions

$$
\ldots, h-\omega, h, h+\omega, \ldots
$$

of eq. (g) ;

$$
h_{t} \rightarrow\left\{x_{s}: s \in \underline{R}\right\}=: 0 \text { as } t \rightarrow-\infty, h_{t}+0+\omega \text { as } t \rightarrow \infty
$$

so that intersections modulo $w$ of $\underline{R} \exists t \rightarrow h_{t} \in C$ with hyperplanes $H$

and $\mathrm{H}+\omega$ define a homoclinic trajectory $\left(x_{n}\right)_{-\infty}^{\infty}$ of an associated Poincare map, with a transversality property (Corollaries 1, 2, 4 in Sec- tion IV.4) which permits to apply Theorem 5.2 [8] (with a minor generalization) and to deduce chaotic behavior.

Alternatively, it is possible to apply Theorem 5.1 [29] which is a bit more comfortable and gives the same result. See Section 6 of [29].

The chaotic solutions of eq. $(g)$ are seen close to the periodic solutions $\ldots, \mathrm{x}-\omega, \mathrm{x}, \mathrm{x}+\omega, \ldots$ and their heteroclinic connections $\ldots, h-\omega, h, h+\omega, \ldots$. For example, there are infinitely many distinct orbits in $\mathrm{C}$ of periodic solutions of the second kind,

$$
\mathrm{y}(\cdot+\mathrm{p})=\mathrm{y}+\mathrm{n} \omega \text { for some } \mathrm{p}>0 \text { and some } \mathrm{n} \in \underline{\underline{N}} \text {. }
$$

Such solutions $y: \underline{R} \rightarrow \underline{R}$ describe periodic motion of the state variable on the circle $\underline{R} \bmod \omega$, composed of rotations around and small oscillations near $x(\underline{R}) \bmod \omega$.

We give an outline of the search for sine-like nonlinearities with the desired properties.

Chapter I deals with unstable periodic solutions of equation

$\left(\operatorname{ag}_{0}\right) \quad \dot{x}(t)=\operatorname{ag}_{0}(x(t-1))$

with parameter $a>0$ and $g_{0}: \underline{R} \rightarrow \underline{R} C^{1}$-smooth, $g_{0}(0)=0, g_{0}^{\prime}(0)=1$. Linearization of the semiflow at $0 \in C$ leads to the "characteristic equation"

((a) $z-a e^{-z}=0$

for the spectrum of the generator. An inspection of this transcendental equation shows that at

$$
a=\frac{3 \pi}{2}=: \alpha
$$

there is a Hopf bifurcation of periodic solutions with periods close to $\frac{4}{3}$. A positive solution $\xi_{\alpha}$ of eq. $((\alpha))$ indicates that the bifurcating periodic orbits have at least one Floquet multiplier outside $\underline{S}$ which makes them unstable.

A major difficulty is to control the second Floquet multiplier close to the multiplier 1 , which is present due to Hopf bifurcation: Is it equal to 1 , inside $\underline{\dot{S}}$ or outside ? There seems to be no Hopf bifurcation theorem in the literature which leads to conditions on $g_{0}$ and its derivatives ensuring that the multiplier close to 1 lies strictly inside $\underline{\mathrm{S}}$.

For this reason, theorems on Hopf bifurcation are abandoned. We impose the conditions that for some $\gamma>0$ 
$g_{0}$ is odd in $[-\gamma, \gamma]$ and

$g_{0}^{\prime}$ is strictly decreasing on $[0, \gamma]$.

This permits to obtain a supercritical curve $\alpha<a \rightarrow x_{a, 0} \in C$ of initial values for bifurcating periodic solutions $x_{a}$, by an idea of Kaplan and Yorke [13] and by a result of Nussbaum [20].

Then we can employ the method from [31], originally developped for the less local problem of bifurcation from periodic orbits, and show that for a $-\alpha>0$ small the second multiplier close to 1 lies strictly inside s.In other words, the solutions $x_{a}$ are hyperbolic with one unstable direction, given by the eigenspace of the multiplier outside $\underline{s}$.

Positive feedback

$$
0<\xi g_{0}(\xi) \text { for } 0<|\xi| \leqq \gamma
$$

and monotonicity imply instability of solutions with values in $[-\gamma, \gamma]$, like $x_{a}$, also in terms of elementary inequalities for arifting away from each other. Section I. 7 contains what is needed for the sequel.

Chapter II is abstract. For a $C^{1}$-map $f$ with a hyperbolic fixed point $x^{*} ;$ local stable and unstable manifolds are introduced as in [8]. (and [18]); $f$ is not necessarily invertible. The final application of Theorem 5.2 [8] on chaos is prepared, and a detailed proof is given for a technical result on motion towards subsets of the local unstable manifold (Proposition II.1.1). The latter helps to link local behavior near $x^{*}$ (where $f$ is dominated by $D f\left(x^{*}\right)$ ) to motion far away.

Section III. 1 introduces Poincare" maps

$$
\mathrm{P}_{\mathrm{a}}: \mathrm{D}_{\mathrm{a}} \rightarrow \mathrm{H}
$$

for the periodic solutions $\mathrm{x}_{a} \cdot \mathrm{D}_{a}$ is an open subset of a hyperplane $\mathrm{H}$ in $c$ which is transversal to the trajectory $t \rightarrow x_{a}, t$ of $x_{a}$ in $c$

at $t=0$. The initial values

$$
x_{a, 0}=: \phi_{a}^{*}
$$

become hyperbolic fixed points of the maps $P_{a}$, with one-dimensional linear unstable space' $\underline{R} \lambda_{a}$.

We know the limiting direction of the linear unstable spaces as $a \psi \alpha$. This permits to fix a $>\alpha$ sufficiently close to $\alpha$ so that one can control the position or the solutions starting in the local unstable manifold of $\phi_{a}^{*}$, in terms of inequalities: we set $g_{1}:=a g_{0}$ and drop the index $a$. Let $U \subset H$ and $S \subset H$ denote the local unstable and local stable manifolds of $\phi^{*}$. Segments $y_{2}$ of solutions of eq. $\left(g_{1}\right)$ with initial value in $U \backslash S$, reach an open cone in $C$ with vertex $\phi^{*}$ (Proposition III.2.1).

The idea how to find a periodic nonlinearity, $g$ with a heteroclinic solution from $x$ to $x+\omega$ is basically as in [32] and in $[10,9]$. It exploits the delay: Let us assume that $g_{1}$ is increasing on all of $\underline{R}^{+}{ }^{-}$. not yet periodic. The instability results from section $I .7$ and Proposition III.2.1 imply that cardidates $h: \underline{R} \rightarrow \underline{R}$ for heteroclinic solutions, with $h_{0}$ in the branch of $U$ above $S$, increase to values $h(t)>\gamma$, with

$$
-\gamma<h<h(t) \text { on }(-\infty, t) \text { and } 0<\dot{h} \text { on }[t, t+1] \text {. }
$$

A change of $g_{1}$ outside the interval $[-\gamma, h(t)]$ would not affect results for the small amplitude solution $x$, for $P$ and $h \mid(-\infty, t+1)$; a modification of $g_{1}$ on the interval $(h(t), h(t+1)]$ can be used to steer the solution $h$ of the delay equation to prescribed values on subintervals of $(t+1, t+2]$.

The program is then to change $g_{1}$ outside $[-\gamma, \gamma]$

to a sine-like function $g$ with minimal period $\omega$

so that a segment $h_{t_{1}}, t_{1}>0$, of a solution $h$ with $h_{0} \in 0$ reaches the local stable manifold $S+\omega \subset D+\omega \subset H+\omega$ of the fixed point $\phi^{*}+\omega$ of the shifted Poincare map on $D+\omega$

- in such a way that a tangent vector $v \in T_{h_{0}} U$ is transported to a vector $\mathrm{v}_{1} \in \mathrm{H}$ transversal to $\mathrm{s}+\mathrm{w}$ at $h_{t_{1}}$.

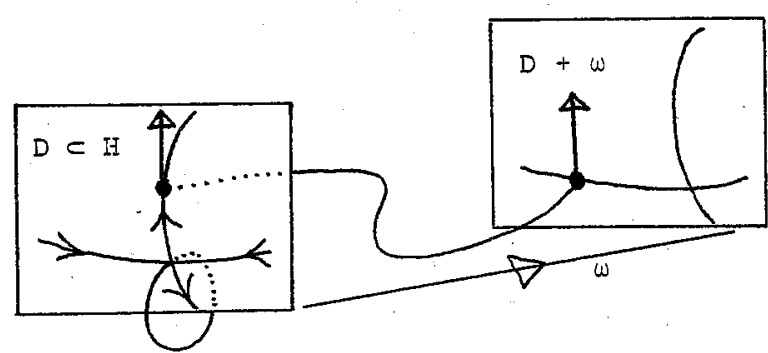


As it can not be expected to hit the subset $s+\omega$ of codimension 1 in $\mathrm{H}+\omega$ immediately, we look for a one-parameter-family of functions $g=g_{b}, b \geq 4$, with solutions $h=h_{b}$ such that $h_{4, t}$ reaches $F+w$ below $s+w$ while for some $b^{*}>4, h_{b^{*}, t}$ reaches $H+w$ above $s+w$ so that continuity implies $h_{b, t_{1}} \in S+\omega$ for some intermediate parameter $b$ :

section III. 3 contains local preparations for this. It' is here that the technical Proposition II.1.1 enters, via Corollary III.2.2\%

Section III.4 characterizes transversality by inequalities for solutions $w$ of the linear variational equation along the heteroclinic solution h,

$\dot{w}(t)=g^{\prime}(h(t-1)) w(t-1)$,

that is, directly in terms of the objects $g, h$ which we are going to construct.

In Chapter IV the program sketched above is carried out. The initial value $X=h_{0} \in U$ becomes a transversal homoclinic point of a modification $P^{\prime}$ of the map $P$ : For $\phi \in H$ close to $X \neq \phi^{*}, P^{\prime}$ is given by intersections of trajectories $t \rightarrow X(t, \phi) \approx h_{t}$ with $H+\omega$ at $t \approx t_{1}$ and by a shift modulo w back into $H$.

Proposition IV. 4.1 exhibits an important difference to the former examples of chaotic differential delay equations $[32,23,10,11,9]$. The heteroclinic solution does not flow into the limiting periodic orbit in firite time. In particular, the points

$$
S \ni\left(P^{\prime}\right)^{n}(X)=h_{t_{n}}-w \text {, with } n \in \underline{N} \text { and } t_{n}>0 \text {, }
$$

are all different from $\phi^{*}$, and the homoclinic sequence is not entirely contained in the one-dimensional submanifold $U$.

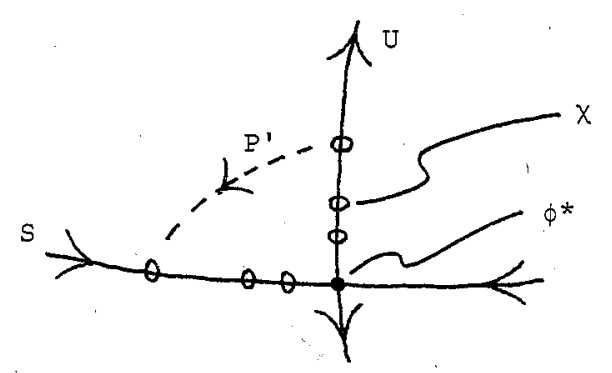

This is in contrast to $[32,10,11]$ where we could reduce the poincare map to a map on a one-dimensional submanifold which contained the unstable fixed point and a homoclinic trajectory jumping on the fixed point at some index -

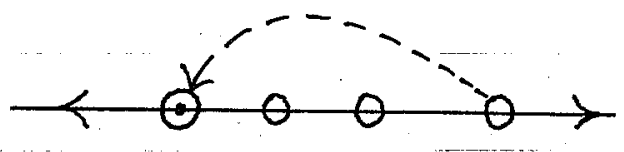

so that chaos followed from Marotto's result on maps in finite dimensional spaces with "snap-back repellers" [16].

Chapter V presents a short application of the work of Hale and Lin [8] on chaos to the map $P^{\prime}$. 


\section{PRELIMINARIES}

The sets of integers, positive integers, real and complex numbers are denoted by $\underline{z}, \underline{\mathbb{N}}, \underline{\mathrm{R}}$ and $\underline{\mathrm{C}}$, respectively. $\underline{\mathbb{N}}_{0}:=\underline{N} \cup\{0\}, \underline{\mathrm{R}}^{+}:=[0, \infty)$ and $\underline{R}^{-}:=(-\infty, 0]$. For $0 \neq x \in \underline{R}, \operatorname{sign} x=\frac{x}{|x|} ; \operatorname{sign} 0:=0 . \underline{S}$ is the unit circle in $\underline{\mathrm{C}}$.

Let $L$ be a real Banach space with norm $\mid$. For $x>0, L_{r}:=$ $\{x \in L:|x|<x\} . I^{C}$ denotes the space of continuous linear maps $I \rightarrow I$. For $A \in I^{C}$, the spectrum $\sigma(A)$ is defined as the spectrum of its complexification $\tilde{\mathrm{A}} \in \tilde{\mathrm{I}}^{\mathrm{C}} ; \tilde{\mathrm{I}}^{\mathrm{C}}$ is the space of continuous $\underline{\mathrm{C}}$-linear maps of the complexified space $\tilde{\mathrm{I}}$ into $\tilde{\mathrm{I}}$.

For a map $f: D \rightarrow L, D \in I$, domains of iterates $f^{\mathbb{M}}, n \in \underline{\mathbb{N}}$, are denoted by $D_{(n)}$; note $D_{(n+1)} \subset D_{(n)} \subset D_{(1)}=D$ for all $n \in \underline{N} \cdot A$ trajectory of $f$ is a sequence $\left(x_{n}\right)_{n \in \underline{Z}}=\left(x_{n}\right)_{-\infty}^{\infty} \in D \underline{Z}$ with $x_{n+1}=f\left(x_{n}\right)$ for all $n \in \underline{z}$. Similarly for forward and backward trajectories $\left(\mathrm{x}_{\mathrm{n}}\right)_{0}^{\infty}$ and $\left(x_{n}\right)_{-\infty}^{0}$.

A fixed point $x^{*}$ of a $C^{1}$-map $f$ with $D$ open is called hyperbolic if $\sigma\left(D f\left(x^{*}\right)\right) \cap \underline{s}=\emptyset$. A homoclinic trajectory $\left(x_{n}\right)_{-\infty}^{\infty}$ of $x^{*}$ is defined by $x_{n} \rightarrow x^{*}$ as $|n| \rightarrow \infty$ and $x_{n} \neq x^{*}$ for some $n$. This includes "snap-back repellers" $x^{*}$ [16] with an index $n$ such that for $j<n$, $x_{j} \neq x^{*}$ while $x_{j}=x^{*}$ for $j \geqq n$.

If $f$ is injective then the inverse $f^{-1}$ is defined as a map from $f(D)$ into $I$.

A tangent to a set $M \subset L$ at a point $x \in M$ is a vector $v \in I$ such that $\mathrm{v}=\mathrm{DC}(0) 1$. for a $C^{1}$-curve $c:(-1,1) \rightarrow \mathrm{L}$ with $c(0)=\mathrm{x}$ and range in $M$. The set of tangents to $M$ at $x \in M$ is denoted by $T x^{M}$.

For $c^{k}$-submanifolds of $i$, we refer to [1].

We collect the basic facts on scalar differential delay equations. Proofs are found in [7], or carried out with the aid of [7].

Let $g: \underline{R} \rightarrow \underline{R}$ be given. A solution of equation

(g) $\dot{x}(t)=g(x(t-1))$ is either a differentiable function $x: \underline{R} \rightarrow \underline{R}$ which satisfies eq. $(g)$ for all $t$, or a continuous function $x:[\underline{t}-1, \infty) \rightarrow \underline{R}$ which is differentiable and satisfies eq. $(g)$ for all $t>\underline{t}$. If $g$ is linear then complexvalued solutions are defined analogously. It is also clear how to define solutions of nonautonomous equations

$$
\dot{x}(t)=A(t) x(t-1)
$$

for $A:[\underline{t}, \infty) \rightarrow \underline{R}$ or $A: \underline{R} \rightarrow \underline{R}$.

Let $C$ denote the Eanach space of continuous functions $[-1,0] \rightarrow \underline{R}$, with the maximum-norm. As complexification of $C$ we consider the space $\tilde{C}$ of continuous functions $[-1,0] \rightarrow \underline{c}$, also with the maximum-norm. Constant functions in $\mathrm{C}$ or $\tilde{\mathrm{C}}$ with value $\xi$ are denoted by $\xi$, too.

Each solution $\mathrm{x}$ of eq. $(g)$ defines a continuous trajectory in $C$ (or in $\tilde{c}$ ) by $x_{t}:=x(t+\cdot) \mid[-1,0]$ for all $t \in \underline{R}$ with $[t-1, t]$ in the domain of $x$.

If the solution carries an index, like $x_{1}$, then we write $x_{1}, t$ for the values of the trajectory.

Let $g$ be continuous, $\underline{t} \in \underline{R}$. Then every $\phi \in C$ determines a unique solution $x:[\underline{t}-1, \infty) \rightarrow \underline{R}$ with $x_{t}=\phi$, and we have continuous dependence on initial data (restrictions of $x$ to compact intervals depend continuously on $\phi$ with respect to uniform convergence). This is most easily seen from the formula

$$
x(t)-x(\underline{t}+n)=s_{\underline{t+n-1}}^{t-1} g \circ x
$$

for $\underline{t}+n \leqq t \leqq \underline{t}+n+1$ and $n \in \underline{N}_{0}$.

Also, if $\tilde{g}: \underline{R} \times \underline{R} \rightarrow \underline{R}$ is continuous, if $t \geqq 0$ and $\phi \in \mathcal{C}$, and if for $b \in \underline{R} \quad x_{b}:[-1, \infty) \rightarrow \underline{R}$ denotes the solution of

$\dot{x}(t)=\tilde{g}(b, x(t-1)), x_{0}=\phi$

then the map $\underline{R} \exists b+x_{b, t} \in C$ is continuous.

suppose $g$ is $C^{k}, k \in \underline{N}_{0}$. Then solutions $x:[\underline{t}-1, \infty)+\underline{R}$ are $C^{k}$ on $(\underline{t}+k, \infty)$.

Solutions $\mathrm{x}=\mathrm{x}^{\phi}$ of initial value problems $\dot{x}(t)=g(x(t-1))$ for $t>0, x_{0}=\phi$

define a continuous semiflow $x=x_{g}, x: \underline{R}^{+} \times C \rightarrow C$ by $x(t, \phi)=x_{t}^{\phi}$ for $t \geqq 0, \phi \in C \cdot x$ is $C^{k}$ on $(k, \infty) \times C$.

For $t \geqq 1$, the maps $X(t, \cdot)$ are compact (bounded sets are mapped 
onto relatively compact sets). This follows by Ascoli's theorem since eq. (g) implies that for $x_{0}=\phi$ in a bounded set and for $t \geqq 0$ given, solutions and their derivatives are bounded on $(0, t]$.

If $g$ is periodic with period $\omega$ and if $x$ is a solution of eq. $(g)$ then $x+\omega, x-\omega$ are solutions as well, and $x(t, \phi+\omega)=x(t, \phi)+\omega$ $x(t, \phi-\omega)=x(t, \phi)-\omega$ on $\underline{R}^{+} \times C$.

Let $g$ be $C^{1}$. Then the derivative $D_{2} X$ exists and is continuous on all of $\underline{R}^{+} \times C$; it is given by solutions of the linear variational equation: If $\phi \in C, t \geqq 0, \tilde{\phi} \in C$ then $D_{2} X(t, \phi) \tilde{\phi}=w_{t}$ where $w:[-1, \infty) \rightarrow \underline{R}$ is the unique solution of the initial value problem

$$
\dot{w}(t)=g^{\prime}\left(x^{\phi}(t-1)\right) w(t-1) \text { for } t>0, w_{0}=\tilde{\phi} \text {. }
$$

We need an easy consequence for parameterized equations. If $g=\mathrm{ag}_{0}$ with parameter $a>0$, and $g_{0}: \underline{R} \rightarrow \underline{R} \quad c^{1}$, and if continuous maps $a \rightarrow \phi_{a}$ and $a+\tilde{\phi}_{a}$ from some interval into $\mathrm{C}$ are given then the map

$$
a \rightarrow D_{2} x_{a g_{0}}\left(2, \phi_{a}\right) \tilde{\phi}_{a}
$$

is also continuous.

Let $\xi \in \underline{R}$ with $g(\xi)=0$ be given. Then $x: \underline{R} \ni t \rightarrow \xi \in \underline{R}$ is a constant solution. $\xi \in C$ becomes a stationary point of $\mathrm{X}_{\mathrm{g}}=\mathrm{X}$. The linear variational equation along $\mathrm{x}$

$$
\dot{w}(t)=g^{\prime}(\xi) w(t-1)
$$

is autonomous, and $(t, \phi) \rightarrow D_{2} X(t, \xi) \phi$ defines a $C_{0}$-semigroup, called the linearization of $x$ at $\xi$. For $t \geq 1$, the operators in this semigroup are compact. The spectrum of its generator consists of eigenvalues of finite algebraic multiplicity; it is characterized by the zeros of the transcendental function $z+z^{\prime}-g^{\prime}(\xi) e^{-z}$ and their orders (note that the Ansatz $w(t)=e^{z t}$ leads to $z-g^{\prime}(\xi) e^{-z}=0$ ). In case $g^{\prime}(\xi) \neq 0$ there are infinitely many such zeros, heal or complex conjugate pairs. Any set of zeros with $R e z>u, u \in \underline{R}$ given, is finite or empty.

Suppose $x: \underline{R} \rightarrow \underline{R}$ is a periodic solution of eq. $(g)$ with $g C^{1}$. Let $p$ be the minimal period. The points of $\sigma\left(D_{2},\left(p, x_{0}\right)\right)$ are called floquet multipliers of $x: z=1$ is always a Floquet multiplier, namely an eigenvalue with eigenvectior $\dot{x}_{0}$. The periodic solution is said to be hyperbolic if $z=1$ is isolatéd and algebraically simple, and if there is no other floquet multiplier on $\underline{s}$.
In case $p \geqq 1 ; D_{2} X\left(p, x_{0}\right)$ is compact.

A heteroclinic solution of eq. $(g)$ from the periodic solution $x$ to a periodic solution $\tilde{x}$ is a solution $h: \underline{R} \rightarrow \underline{R}$ such that $h_{t}$ converges to the periodic orbit $\left\{\mathrm{x}_{\mathrm{s}}: s \in \underline{\mathrm{R}}\right\}$ as $t \rightarrow-\infty$, and $h_{t} \rightarrow\left\{\tilde{x}_{s}: s \in \underline{R}\right\}$ as $t \rightarrow \infty$.

References in the sequel are made as in the fellowing example.

Lemma $\left\{\begin{array}{l}1 \\ 1.1 \\ \text { I.1.1 }\end{array}\right\}$ means Lemma 1 in $\left\{\begin{array}{l}\text { the same section } \\ \text { Section } 1 \text { of the same chaptex } \\ \text { Section } 1 \text { of Chapter I }\end{array}\right\}$. 
I. HYPERBOLIC PERIODIC SOIUTIONS

Hypotheses and summary. We consider solutions of equations

$\left(a_{0}\right) \quad \dot{x}(t)=a g_{0}(x(t-1))$

with parameter $a>0$ and with a $c^{1}$-function $g_{0}: \underline{R} \rightarrow \underline{R}$. We assume that $g_{0}$ has the following properties.

(H1) $g_{0}(0)=0, g_{0}^{\prime}(0)=1$. There exists $\gamma>0$ such that $0<g_{0}^{\prime}$ on $[-\gamma, \gamma]$ and $g_{0}(\xi)=-g_{0}(-\xi)$ for $|\xi| \leqq \gamma$

and

(H2) $g_{0}^{\prime}$ is strictly decreasing on $[0, \gamma]$.

It is shown that there is a family of periodic solutions $x_{a}$, $a \gtrsim \frac{3 \pi}{2}=: \alpha$, bifurcating from zero such that each $x_{a}$ is hyperbolic with precisely one Floquet multiplier outside $\underline{S}$.

As a link between local and global behavior of solutions, we provide elementary comparison results for solutions not far from $x_{a}$.

\section{PERIODIC SOLUTIONS}

We follow an idea of Kaplan and Yorke [13] and employ a result of Nussbaum [20].

There exists $n^{*}<0$ such that for $n^{*} \leqq \eta<0$, the solution $\left(\mathrm{x}_{\eta}, \mathrm{y}_{n}\right)$ of the Hamiltonian system

$$
\dot{x}=g_{0}(y), \dot{y}=-g_{0}(x)
$$

with $x_{\eta}(0)=0, y_{\eta}(0)=n$. is periodic with minimal period $4 \mathrm{~T}_{\eta}$, and $\eta<y_{n}(t)<0$ in $\left(0, T_{n}\right), y_{n}\left(T_{n}\right)=0,\left|x_{n}(t)\right| \leqq \gamma$ and $\left|y_{n}(t)\right| \leqq \gamma$ for all $t \in \underline{R}$. Moreover, $y_{n}=x_{n}\left(\cdot+T_{n}\right)$ since $g_{0}$ is odd on $[-\gamma, \gamma]$.

(H2) implies that $\xi \rightarrow \frac{g_{0}(\xi)}{\xi}$ is strictly decreasing on $(0, \gamma]$. Therefore Theorem $1.3[20]$ applies (One has to interchange $\mathrm{x}$ and $\mathrm{y}$, and must formulate Theorem 1.3 [20] for initial values with $n$ negative). We conclude that the map $n \rightarrow T_{\eta}$ is strictly decreasing with. $T_{\eta}+\frac{\pi}{2}$ as $n+0$.

Let $a \in\left(\frac{3 \pi}{2}, 3 \mathrm{~T}_{n^{*}}\right]=\left(\alpha, 3 \mathrm{~T}_{n^{*}}\right]$. With $n \in\left[n^{*}, 0\right)$ given by $T_{\eta}=\frac{a}{3} \in\left(\frac{\pi}{2}, T_{n^{*}}\right]$, define $x_{a}(t):=x_{\eta}(a t)$ for all $t \in \underline{R}$. Then

$\dot{x}_{a}(t)=a \dot{x}_{\eta}(a t)=a g_{0}\left(y_{\eta}(a t)\right)=a g_{0}\left(x_{n}\left(a t+T_{\eta}\right)\right)$

$=a g_{0}\left(x_{\eta}\left(a t-3 T_{\eta}\right)\right)=a g_{0}\left(x_{\eta}(a(t-1))\right)$

$$
=\operatorname{ag}_{0}\left(x_{a}(t-1)\right)
$$

for all $t \in \underline{R}$.

Observe $x_{a}(0)=0, \dot{x}_{a}(0)<0$, and $0<x_{a}$ in $\left(-\frac{2}{3}, 0\right), x_{a}<0$ in $\left[-1,-\frac{2}{3}\right)$, and the symmetry

((s)) $x_{a}(t)=-x_{a}\left(t-\frac{2}{3}\right)$ for all $t \in \underline{R}$

so that $x_{a}$ has period $\frac{4}{3} \cdot \dot{x}_{a}$ is strictly decreasing on $\left(-\frac{1}{3}, \frac{1}{3}\right)$.

Set $a_{1}:=3 T_{n *}>\alpha$, and $\phi_{a}^{*}:=x_{a, 0}$ for $\alpha<a<a_{1}$. The map

$a \rightarrow \phi_{a}^{*}$ into $C$ is continuous, with limit $0 \in C$ as $a+\alpha$. 


\section{IINEARIZATION}

For the investigation of Floquet multipliers we collect a few properties of the spectrum of the generator of the linearization at 0 of the semiflow given by eq. $\left(\alpha g_{0}\right)$. These properties are also part of the hypotheses for Hopf bifurcation at $a=\alpha$. One can show that the periodic solutions $\mathrm{x}_{\mathrm{a}}$ arise in a Hopf bifurcation, but we do not pursue this. One reason is that there seems to be no Hopf bifurcation theorem in the literature which allows to derive all we need about the solutions $x_{a}$. - In this respect it might also be noted that $c^{1}$-smoothness as above is a very weak assumption on the nonlinearity $g_{0}$.

Linearizing at zero for $a=\alpha$, we obtain the $C_{0}$-semigroup of operators $\phi \rightarrow y_{t}, t \geqq 0$, where $y:[-1, \infty) \rightarrow \underline{R}$ is the solution of

(a) $\dot{y}(t)=\alpha Y(t-1)$

with $y_{0}=\phi$. For the spectrum of its generator $G$ we have

PROPOSITION 1. All zeros of $E: \subseteq \underline{C} z \rightarrow z-\alpha e^{-z} \in \underline{C}$ are simple. There is exactly one zero $\xi_{\alpha} \in \underline{R} . \xi_{\alpha}$ is positive. $\mathrm{E}\left(\frac{3 \pi i}{2}\right)=0=\mathrm{E}\left(-\frac{3 \pi \dot{i}}{2}\right)$. For ali $z \in E^{-1}(0)-\left\{\xi_{\alpha}, \frac{3 \pi i}{2},-\frac{3 \pi i}{2}, \cdots, \operatorname{Re} z<0\right.$.

PROOF. $E(z)=0=E^{\prime}(z)=1+\alpha e^{-z}$ would imply $z=-1$, a contradiction to $0=E^{\prime}(z)$.

Existence of a unique zero $\xi_{\alpha} \in \underline{R}$ is obvious, as well as $\xi_{\alpha}>0$ and $E\left(\frac{3 \pi i}{2}\right)=0=E\left(-\frac{3 \pi i}{2}\right)$.

We show $z=\frac{3 \pi i}{2}$ for any zero $z=u+i v, u \geq 0$ and $v \geq 0$, with $z \neq \xi_{\alpha}$ : By uniqueness of $\xi_{\alpha}, v>0$. Then $0<v=\alpha e^{-u} \sin (-v) \leq \alpha$ $=\frac{3 \pi}{2}$. In case $0<v<\frac{3 \pi}{2}, 0 \leqq u=\alpha e^{-u} \cos v$ yields $0<v<\frac{\pi}{2}$, or $v=\alpha e^{-u} \sin (-v)<0$, a contradiction. Hence $v=\frac{3 \pi}{2}$.

By $\frac{3 \pi}{2}=\frac{3 \pi}{2} e^{-u \cdot 1}, u=0$.

Let $w$ denote the operator $\phi \rightarrow y_{\frac{2}{3}}$, with the solution $y$ of the initial value problem $\dot{y}(t)=\alpha y(t-1)$ for $t>0, y_{0}=\phi$ as above.
This map is not compact but the iterate $\mathrm{V}_{\alpha}:=\mathrm{w}: \mathrm{W}$, i.e. the $\frac{4}{3}$-translation along solutions of eq. $(\alpha)$, is. It follows that $\sigma(W)$ has the same properties as the spectrum of a compact operator : Each nonzero point $z$ in $\sigma(W)$ is isolated and a pole of the resolvent

$$
\underline{c}-\sigma(W) \exists \zeta \rightarrow(\tilde{W}-\zeta)^{-1} \in \tilde{C}^{c} \text {. }
$$

The generalized eigenspace (i.e., the image of the eigenprojection associated with the spectral set $\{z\}$ ) coincides with $\operatorname{kex}(\tilde{W}-z)^{n}$

where $n \in \underline{N}$ is the order of the pole. We have $\operatorname{ker}(\tilde{W}-z)^{k+1}=\operatorname{ker}(\tilde{W}-z)^{k}$ for $n \leqq k \in \underline{N}$, $\operatorname{ker}(\tilde{W}-z)^{k+1} \underset{\neq}{\supset} \operatorname{ker}(\tilde{W}-z)^{k} \underset{\neq}{\supset}\{0\}$ for $1 \leqq k<n$.

In particular, $\quad z$ is an eigenvalue with finite algebraic multiplicity $\operatorname{dim} \operatorname{ker}(\tilde{w}-z)^{n}$.

For proofs, see e.g. Theorem 6 in VII.4.5 [4] and Theorem 18 in VII.3 [4]. Using Lemma 4.1 of Section 7.4 [7] we infer $\exp \left(\frac{2}{3} \sigma(G)\right) \subset \sigma(W)-\{0\} \in \sigma(W) \subset \exp \left(\frac{2}{3} \sigma(G)\right) \cup\{0\}$

and for $0 \neq z \in \sigma(W)$ and $k \in \underline{\mathbb{N}}$,

$$
\operatorname{ker}(\tilde{W}-z)^{k}=z^{\prime} \in \sigma(G): z=\exp \left(\frac{2}{3} z^{\prime}\right) \operatorname{ker}^{-}\left(\tilde{G}-z^{\prime}\right)^{k} .
$$

COROLLARY 1. The eigenvalue -1 of $w$ has algebraic multiplicity 2 , the eigenvalue $\exp \left(\frac{2}{3} \xi_{\alpha}\right)>1$ of $\mathrm{W}$ has algebraic multiplicity 1 . There exists $\varepsilon \in(0,1)$. with $\sigma(W) \backslash\left\{-1, \exp \left(\frac{2}{3} \xi_{\alpha}\right)\right\} \subset \underline{C}_{1-\varepsilon}$.

Observe that $\psi_{\alpha}:[-1,0] \ni t \rightarrow \exp \left(\frac{4}{3} \xi_{\alpha} t\right) \in \underline{R}$ is an eigenfunction for the eigenvalue $\exp \left(\frac{4}{3} \xi_{\alpha}\right)$ of $v_{\alpha}$. This is most easily seen directly from eq. $(\alpha)$. 
the last case, $\xi_{a}^{*} \in \underline{R}$ and $m_{a}\left(\xi_{a}^{*}\right)=1$. For all $z$ in

$\sigma\left(w_{a}\right)-\left(\left(-1+\underline{C}_{\varepsilon}\right) \cup\left(\exp \left(\frac{2}{3} \xi_{\alpha}\right)+\underline{C}_{\varepsilon}\right)\right),|z|<1-\varepsilon$.

3. TRANSLATION BY VARIATIONAL EQUATIONS

ALONG PERIODIC SOLUTIONS

For $a \in\left(a, a_{1}\right)$, let $w_{a}$ and $v_{a}$ denote the translations along trajectories given by $\phi \rightarrow y_{\frac{2}{3}}$ and $\phi \rightarrow y_{\frac{4}{3}}$, respectively, where

$y:[-1, \infty) \rightarrow \underline{R}$ is the solution of the linear variational equation of $x_{a}$ (a) $\dot{y}(t)=a g_{0}^{\prime}\left(x_{a}(t-1)\right) y(t-1)$

with $y_{0}=\phi \in C$. We are interested in the Floquet multipliers, i.e., in $\sigma\left(v_{a}\right) \cdot \dot{x}_{a, 0}:=\dot{x}_{a} \mid[-1,0] \in C$ is an eigenvector for the eigenvalue 1 of $\mathrm{v}_{a}$. The symetry ((s)) from section 1 implies that $\dot{x}_{a, 0}$ is also an eigenvector for the eigenvalue -1 of $\mathrm{w}_{\mathrm{a}}$, and moreover

(1) $v_{a}=w_{a} \circ W_{a}$.

$\mathrm{V}_{\mathrm{a}}$ is compact. As in section 2 we infer that both sets $\sigma\left(\mathrm{W}_{a}\right) \backslash\{0\}$, $\sigma\left(V_{a}\right)-\{0\}$ consist of isolated points which are all eigenvalues with finite algebraic multiplicities. The eigenvalues are real or occur in complex conjugate pairs.

With the spectral Mapping Theorem in mind, we first study $\sigma\left(\mathrm{W}_{\mathrm{a}}\right)$. For $0 \neq z \in \sigma\left(w_{a}\right)$, let $m_{a}(z)$ denote the algabraic multiplicity. For $z$ in $\underline{c}-\sigma\left(w_{a}\right), m_{a}(z):=0$.

Uniform convergence of $x_{a}$ to 0 on $\left[-1, \frac{2}{3}\right]$ as $a+\alpha$ implies

(2) $\mathrm{w}_{\mathrm{a}}+\mathrm{W}$ in $\mathrm{C}^{\mathrm{C}}$ as $\mathrm{a}+\alpha$.

Results on continuous dependence of spectra (Chapter IV \& 3 no 5 [14]) now yield

COROLIARY 1. There exist $a_{2} \in\left(\alpha, a_{1}\right)$ and $\varepsilon \in(0,1)$ with the following properties.

(i) $\quad$ cl $\underline{c}_{1} \cap\left(\exp \left(\frac{2}{3} \xi_{\alpha}\right)+\underline{c}_{\varepsilon}\right)=\emptyset=\left(-1+\underline{c}_{\varepsilon}\right) \cap\left(\exp \left(\frac{2}{3} \dot{\xi}_{\alpha}\right)+\underline{c}_{\varepsilon}\right)$.

(ii) Let $a \in\left(\alpha, a_{2}\right) \ldots \exp \left(\frac{2}{3} \xi_{\alpha}\right)+\underline{C}_{\varepsilon}$ contains precisely one point $\xi_{a} \in \sigma\left(w_{a}\right)$. We have $\xi_{a}>1$ and $m_{a}\left(\xi_{a}\right)=1$. Either $\sigma\left(W_{a}\right) \cap\left(-1+\underline{C}_{\varepsilon}\right)=\{-1\}$ with $m_{a}(-1)=2$, or $m_{a}(-1)=1$ and $\left(-1+\underline{C}_{\varepsilon}\right) \backslash\{-1\}$ contains precisely one point $\xi_{a}^{*} \in \sigma\left(W_{a}\right)$. In (iii) $\xi_{a} \rightarrow \exp \left(\frac{2}{3} \xi_{\alpha}\right)$ as a $\downarrow \alpha$.

Our aim is to exclude $\mathrm{m}_{\mathrm{a}}(-1)=2$ for each $\mathrm{a}>\alpha$ sufficiently close to $\alpha$, and to show $-1<\xi_{a}^{*}<-1+\varepsilon$ for the other eigenvalue in $-1+\underline{C}_{\varepsilon}$. This will be achieved in Corollary 5.1 below. 
$\left(\begin{array}{ll}u_{1}^{z, a} & u_{2}^{z, a} \\ v_{1}^{z, a} & v_{2}^{z, a}\end{array}\right): \underline{\underline{R}} \rightarrow \underline{\underline{C}}^{2 \times 2}$ of the linear system

4. EIGENVALUES AS ZEROS OF ANALYTIC FUNCTIONS

Let $a \in\left(\alpha, a_{2}\right)$ in this section.

We proceed as in [31] and introduce a function $q_{a}$ whose zeros coincide with $\sigma\left(w_{2}\right) \backslash\{0\}$. The basic idea for this is as follows. Suppose $z \neq 0$ is an eigenvalue of $w_{a}$ with eigenvector $\phi$. Let $w$ denote the corresponding solution of the variational equation along $\mathrm{x}_{\mathrm{a}}$. Define functions on $\left[-\frac{1}{3}, 0\right]$ by

$$
\begin{aligned}
& \phi_{1}:=\phi\left(-\frac{2}{3}+\cdot\right), \phi_{2}:=\phi\left(-\frac{1}{3}+\cdot\right), \phi_{3}:=\phi(\cdot), \\
& w^{*}:=w\left(\frac{1}{3}+\cdot\right), w^{* *}:=w\left(\frac{2}{3}+\cdot\right) .
\end{aligned}
$$

In terms of these functions, $w_{\frac{2}{3}}=w_{a} \phi=z \phi$ reads

$$
\phi_{3}=z \phi_{1}, w^{*}=z \phi_{2}, w^{* *}=z \phi_{3} .
$$

By the variational equation,

$$
\begin{aligned}
\dot{w}^{* *}(\cdot) & =\dot{w}\left(\frac{2}{3}+\cdot\right)=\operatorname{ag}_{0}^{\prime}\left(x_{a}\left(\frac{2}{3}+\cdot-1\right)\right) w\left(\frac{2}{3}+\cdot-1\right) \\
& =\operatorname{ag}_{0}^{\prime}\left(x_{a}\left(-\frac{1}{3}+\cdot\right)\right) \phi_{2}(\cdot)=\frac{1}{z} \operatorname{ag}_{0}^{\prime}(\ldots) w^{*}(\cdot), \\
\dot{w}^{*}(\cdot) & =\dot{w}\left(\frac{1}{3}+\cdot\right)=\operatorname{ag}_{0}^{\prime}\left(x_{a}\left(\frac{1}{3}+\cdot-1\right)\right) w\left(\frac{1}{3}+\cdot-1\right) \\
& \operatorname{ag}_{0}^{\prime}\left(x_{a}\left(-\frac{2}{3}+\cdot\right) \phi_{1}^{\prime}(\cdot)=\operatorname{ag}_{0}^{\prime}(\ldots) \frac{1}{z} \phi_{3}(\cdot)=\frac{1}{z^{2}} \operatorname{ag}_{0}^{\prime}(\ldots) w^{* *}(\cdot)\right.
\end{aligned}
$$

The continuity of $w$ implies boundary conditions

$$
w^{*}\left(-\frac{1}{3}\right)=\phi_{3}(0)=\frac{1}{2} w^{* *}(0) \text { and } w^{*}(0)=w^{* *}\left(-\frac{1}{3}\right),
$$

or

$$
\left(\begin{array}{l}
w^{* *} \\
w^{*}
\end{array}\right)(0)=\left(\begin{array}{ll}
0 & z \\
1 & 0
\end{array}\right) \cdot\left(\begin{array}{l}
w^{* *} \\
w^{*}
\end{array}\right)\left(-\frac{1}{3}\right)
$$

The left hand side is the product of the vector on the right hand side with the fundamental matrix solution of the above system, which equals the identity at $t=-\frac{1}{3}$. So, if $w^{* *}\left(-\frac{1}{3}\right)$ and $w^{*}\left(-\frac{1}{3}\right)$ are not both zero a determinant must vanish at $z$.

It is now clear how to define the function $q_{a}$ :

For $t \in \underline{R}$, set

$$
A_{a}(t):=a g_{0}^{\prime}\left(x_{a}\left(t-\frac{1}{3}\right)\right), B_{a}(t):=a_{0}^{\prime}\left(x_{a}\left(t-\frac{2}{3}\right)\right)=a g_{0}^{\prime}\left(x_{a}^{\prime}(t)\right) \text {. }
$$

For $0 \neq z \in \underline{C}$, let $S_{z, a}$ denote the fundamental matrix solution

$$
\dot{\mathrm{u}}=\frac{1}{\mathrm{z}} \mathrm{A}_{\mathrm{a}}(\mathrm{t}) \mathrm{V}, \dot{\mathrm{v}}=\frac{1}{\mathrm{z}^{2}} \mathrm{~B}_{\mathrm{a}}(\mathrm{t}) \mathrm{u}
$$

which is equal to the identity matrix at $t=-\frac{1}{3}$. Set

$$
Q_{z, a}:=s_{z, a}(0)-\left(\begin{array}{ll}
0 & z \\
1 & 0
\end{array}\right)=\left(\begin{array}{ll}
u_{1}^{z, a}(0) & u_{2}^{z, a}(0)-z \\
v_{1}^{z, a}(0)-1 & v_{2}^{z, a}(0)
\end{array}\right)
$$

and $q_{a}(z):=\operatorname{det} Q_{z, a}$. The function $q_{a}: \underline{c}-\{0\}+\underline{c}$ is analytic (see e.g. Section $x .7$ [3]). From det $s_{z, a}(t)=1$ on $\underline{R}$, we infer

$$
q_{a}(z)=1-z+u_{2}^{z, a}(0)+z \cdot v_{1}^{z, a}(0) \text { for all } z \in \underline{C}-\{0\} \text {. }
$$

In order to establish relations between the zeros of $q_{a}$ and eigenvalues of $\mathrm{w}_{\mathrm{a}}$ we need a description of the operators $\tilde{\mathrm{w}}_{\mathrm{a}}-\mathrm{z}, 0 \neq \mathrm{z} \in \underline{\mathrm{C}}$, in terms of solutions of inhomogeneous boundary value problems.

It is convenient to introduce the Banach space $C^{\prime}$ of triples $\left(\phi_{1}, \phi_{2}, \phi_{3}\right)$ of continuous functions $\left[-\frac{1}{3}, 0\right] \rightarrow \subseteq$ satisfying

$$
\phi_{1}(0)=\phi_{2}\left(-\frac{1}{3}\right), \phi_{2}(0)=\phi_{3}\left(-\frac{1}{3}\right),
$$

with norm given by $\left|\left(\phi_{1}, \phi_{2}, \phi_{3}\right)\right|=\max _{j} \max _{t}\left|\phi_{j}(t)\right|$.

Let $J$ denote the norm-preserving isomorphism from $\tilde{C}$ onto $\mathrm{C}^{\prime}$ defined by $J \phi=\left(\phi_{1}, \phi_{2}, \phi_{3}\right)$, with the restrictions $\phi_{1}, \phi_{2}, \phi_{3}$. of $\phi\left(-\frac{2}{3}+\cdot\right), \phi\left(-\frac{1}{3}+\cdot\right), \phi$ to the interval $\left[-\frac{1}{3}, 0\right]$.

Let $0 \neq z \in \underline{C}, \chi \in \tilde{C}, \phi \in \tilde{C}$. One sees, as at the beginning of the section, that $\left(\tilde{w}_{a}-z\right) X=\phi$ implies

(1) $\quad \dot{u}=\frac{1}{z} A_{a}(t) \ldots\left[v-\phi_{2}\right]$

$$
\dot{v}=\frac{1}{z^{2}} B_{a}(t)\left[u-\phi_{3}\right]-\frac{1}{z} B_{a}(t) \phi_{1}
$$

where $u:=\left(\tilde{w}_{a} x\right)_{3}, v:=\left(\tilde{w}_{a} x\right)_{2}$ and

$$
\begin{aligned}
& v(0)=u\left(-\frac{1}{3}\right), \\
& u(0)=\tilde{w}_{a} X(0)=\phi(0)+z X(0)=\phi_{3}(0)+z x_{3}(0)=\phi_{3}(0)+z \cdot v\left(-\frac{1}{3}\right) .
\end{aligned}
$$

By variation of constants,

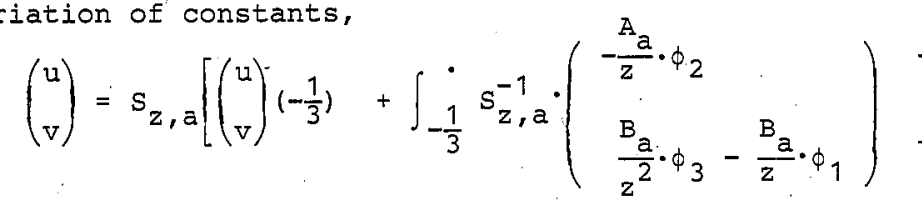

Let $I(z, a, \phi, t)$ denote the last integral at $t \in\left[-\frac{1}{3}, 0\right]$, with components $I_{1}(\ldots)$ and $I_{2}(\ldots)$. 
Set $\quad c:=\left(\begin{array}{l}c_{1} \\ c_{2}\end{array}\right):=\left(\begin{array}{l}u \\ v\end{array}\right)\left(-\frac{1}{3}\right) \cdot$. Then $\left(\begin{array}{c}\phi_{3}(0)+z \cdot c_{2} \\ c_{1}\end{array}\right)=\left(\begin{array}{c}\phi_{3}(0)+z \cdot v\left(-\frac{1}{3}\right) \\ u\left(-\frac{1}{3}\right)\end{array}\right)$

$=\left(\begin{array}{l}u \\ v\end{array}\right)(0)=s_{z, a}(0) c+s_{z, a}(0) \cdot I(z, a, \phi, 0)$, or

$$
-Q_{z, a} \cdot c=-\left[S_{z, a}(0)-\left(\begin{array}{ll}
0 & z \\
1 & 0
\end{array}\right)\right] \cdot c=I_{z, a} \Phi-
$$

with the linear continuous map

$$
I_{z, \dot{a}}: \tilde{C} \ni \phi \rightarrow\left(\begin{array}{c}
-\phi_{3}(0) \\
0
\end{array}\right)+S_{z, a}(0) \cdot I(z, a, \phi, 0) \in \underline{C}^{2} .
$$

We have shown the first part of

IEMMA 1. Let $0 \neq z \in \underline{c}, \phi \in \tilde{C}$. If $x \in \tilde{c}$ and $\left(\tilde{w}_{a}-z\right) x=\phi$ then $(u, v)$ definea by $J \tilde{W}_{a} X=\left(x_{3}, v, u\right)$ satisfies eq. (1) for all $t \in\left[-\frac{1}{3}, 0\right]$ and $-Q_{z, a} \cdot\left(\begin{array}{l}u \\ v\end{array}\right)\left(-\frac{1}{3}\right)=I_{z, a} \phi$.

Conversely, if $c \in \underline{c}^{2}$ and

(*) $-Q_{z, a} \cdot c=L_{z, a} \phi$,

and if $(u, v)$ is the restriction of the solution of eq. (1) with

$\left(\begin{array}{l}u \\ v\end{array}\right)\left(-\frac{1}{3}\right)=c$ to the interval $\left[-\frac{1}{3}, 0\right]$, then

$\frac{1}{z}\left(\frac{1}{z}\left(u-\phi_{3}\right)-\phi_{1}, v-\phi_{2}, u-\phi_{3}\right) \in C^{\prime}$,

and $x:=J^{-1}\left(\frac{1}{2}(\ldots)\right) \in \tilde{C}$ satisfies $\left(\tilde{W}_{a}-z\right) x=\phi$.

In case $\phi=0$ and $c \neq 0, x \neq 0$.

PROOF of the second part. Let $c \neq 0$ with $(*)$ be given.

1. For $t \in\left[-\frac{1}{3}, 0\right]$, set

$$
\left(\begin{array}{l}
u \\
v
\end{array}\right)(t):=s_{z, a}(t)[c+I(z, a, \phi, t)]
$$

and $x_{3}:=\frac{1}{z}\left(u-\phi_{3}\right), x_{2}:=\frac{1}{z}\left(v-\phi_{2}\right), x_{1}:=\frac{1}{z}\left(x_{3}-\phi_{1}\right)$. Then

$$
x_{3}-z x_{1}=\phi_{1}, v-z x_{2}=\phi_{2}, u-z x_{3}=\phi_{3} .
$$

2. We have $\left(x_{3}, v, u\right)=J \tilde{x}$ with $\tilde{x} \in \tilde{c}$. Proof: By definition of $u$ and $v, u\left(-\frac{1}{3}\right)=c_{1}$ and $v\left(-\frac{1}{3}\right)=c_{2} \cdot B y(*)$ and by definition of $u$,

$$
z c_{2}=c_{1} u_{1}^{z, a}(0)+c_{2} u_{2}^{z, a}(0)+u_{1}^{z, a}(0) \cdot I_{1}(z, a, \phi, 0)+u_{2}^{z ; a}(0) \cdot I_{2}(\ldots)
$$

$$
-\phi_{3}(0)=u(0)-\phi_{3}(0) .
$$

Similarly, $\quad c_{1}=\ldots=v(0)$. Together, $v(0)=u\left(-\frac{1}{3}\right)$, and

$$
v\left(-\frac{1}{3}\right)=\frac{1}{2}\left(u(0)-\phi_{3}(0)\right)=x_{3}(0) \text {. }
$$

Recall the definition of $\mathrm{J}$.

3. By linearity, $\left(x_{1}, x_{2}, x_{3}\right)=J x$ with $x \in \tilde{c}$.

4. In order to verify. $\left(\tilde{w}_{a}-z\right) x=\phi$, ox equivalently

$$
J\left(\tilde{w}_{2}-z\right) x=\left(\phi_{1}, \phi_{2}, \phi_{3}\right) \text {, }
$$

note first $\left(\tilde{w}_{a} x\right)_{1}=x_{3}$ so that $\left(\left(\tilde{W}_{a}-z\right) x\right)_{1}=x_{3}-z x_{1}=\phi_{1}$.

Next, $\left(\tilde{w}_{\mathrm{a}} \mathrm{x}\right)_{2}=\mathrm{w}\left(\frac{1}{3}+\cdot\right) \mid\left[-\frac{1}{3}, 0\right]$ where $\mathrm{w}:[-1, \infty)+\underline{\mathrm{c}}$ is the solution

of

$$
\dot{w}(t)=a g_{0}^{\prime}\left(x_{a}(t-1)\right) w(t-1), w_{0}=x .
$$

on $\left(-\frac{1}{3}, 0\right]$ we obtain

$$
\begin{aligned}
\dot{w}\left(\frac{1}{3}+\cdot\right) & =B_{a} x_{1}=\frac{1}{z} B_{a} z x_{1}=\frac{1}{z} B_{a}\left[x_{3}-\phi_{1}\right]=\frac{1}{z^{2}} B_{a}\left[u-\phi_{3}\right]-\frac{1}{z} B_{a} \phi_{1} \\
& =\dot{v} .
\end{aligned}
$$

with $w\left(\frac{1}{3}-\frac{1}{3}\right)=w(0)=x_{3}(0)=v\left(-\frac{1}{3}\right), w\left(\frac{1}{3}+\cdot\right)=v=z x_{2}+\phi_{2}$ on the interval $\left[-\frac{1}{3}, 0\right]$, or $\left(\tilde{w}_{\mathrm{a}} x\right)_{2}-z x_{2}=\phi_{2}$.

Finally, $\left(\tilde{\mathrm{w}}_{\mathrm{a}} \mathrm{x}\right)_{3}=\mathrm{w}\left(\frac{2}{3}+\cdot\right) \mid\left[-\frac{1}{3}, 0\right]$, and on the interval $\left[-\frac{1}{3}, 0\right]$,

$$
\dot{\mathrm{w}}\left(\frac{2}{3}+\cdot\right)=\mathrm{A}_{\mathrm{a}} \mathrm{X}_{2}=\frac{1}{\mathrm{z}} \mathrm{A}_{\mathrm{a}}\left[\phi_{2}-z \mathrm{x}_{2}\right]-\frac{1}{\mathrm{z}} \mathrm{A}_{\mathrm{a}} \phi_{2}=\frac{1}{2} \mathrm{~A}_{\mathrm{a}} \mathrm{v}-\frac{1}{\mathrm{z}} \mathrm{A}_{\mathrm{a}} \phi_{2}=\dot{\mathrm{u}} .
$$

with $u\left(-\frac{1}{3}\right)=v(0)=w\left(\frac{1}{3}\right)=w\left(\frac{2}{3}-\frac{1}{3}\right)$, we get

$$
u=w\left(\frac{2}{3}+\cdot\right) \mid\left[-\frac{1}{3}, 0\right],
$$

and therefore $\left(\tilde{w}_{a} X\right)_{3}=u=z x_{3}+\phi_{3}$.

5. The last assertion in the lemma is obvious.

COROLLARY 1. Let $0 \neq z \in \underline{c}$. Then $z \in \sigma\left(W_{a}\right)$ if and only if $q_{a}(z)=0$. PROOF. Each nonzero $z \in \sigma\left(W_{a}\right)$ is an eigenvalue. We saw at the begin of the section that for eigenvalues $z \neq 0, q_{a}(z)=0$. Conversely, if $q_{a}(z)=0$ then there exists $c \in \underline{c}^{2}-\{0\}$ with $-Q_{z, a} c=0$. By Lerma 1 , with $\phi=0$, there is. $x \in \tilde{C} \backslash\{0\}$ with $\left(\tilde{w}_{a}-z\right) x=0$. Hence $z$ is in $\sigma\left(w_{2}\right)$

The next objective is to show that algebraic multiplicities coincide with the order $j_{a}(z)$ of $z \in \underline{C}-\{0\}$, considered as a zero of the analytic function $q_{a}$. We follow [31]. Details are a bit more involved. 
PROPOSITION 1. The operators $\mathrm{L}_{z, a}, 0 \neq z \in \underline{C}$, are surjective.

PROOF. 1. There is a sequence of points $\phi^{(n)} \in \tilde{c}$ such that $\phi^{(n)}=1$ for all $n \in \underline{N}$ and $I_{z, a^{(n)}} \rightarrow\left(\begin{array}{l}1 \\ 0\end{array}\right)$ as $n \rightarrow \infty$. So, vectors close to $\left(\begin{array}{l}1 \\ 0\end{array}\right)$ are contained in $\mathrm{L}_{z, a} \tilde{c}$. We look for linearly independent vectors in $I_{z, a} \tilde{c}$.

2. There exists $\phi \in \tilde{\mathrm{C}}$ with $\phi_{1}=0=\phi_{3}$ such that the second component of

$$
c:=I_{z, a^{\phi}}=S_{z, a}(0) \int_{-\frac{1}{3}}^{0} \frac{1}{z} A_{a} \phi_{2}\left(\begin{array}{c}
-v_{2}^{z, a} \\
z, a \\
v_{1}
\end{array}\right)
$$

does not vanish. Proof: For $\psi$ with $\psi_{1}=0=\psi_{3}$

$$
z\left(I_{z, a} \psi\right)_{2}=v_{1}^{z, a}(0) \int_{-\frac{1}{3}}^{0} A_{a} \psi_{2}\left(-v_{2}^{z, a}\right)+v_{2}^{z, a}(0) \int_{-\frac{1}{3}}^{0} A_{a} \psi_{2} v_{1}^{z, a}
$$

In case $v_{2}^{z, a}(0)=0$, det $s_{z_{r}, a}(0)=1$ gives $v_{1}^{z, a}(0) \neq 0 \cdot A_{a}>0$ and $v_{2}^{z, a}\left(-\frac{1}{3}\right)=1$ allow to find $\phi$ with $\phi_{1}=0=\phi_{3}$ such that $\left(I_{z, a} \phi\right)_{2} \neq$ 0 .

In case $v_{1}^{z, a}(0)=0, v_{2}^{z, a}(0) \neq 0$. Now $v_{1}^{z, a}\left(-\frac{1}{3}\right)=0$. But

$$
\dot{v}_{1}^{z, a}\left(-\frac{1}{3}\right)=\frac{1}{z^{2}} B_{a}\left(-\frac{1}{3}\right) u_{1}^{z, a}\left(-\frac{1}{3}\right)=\frac{1}{z^{2}} B_{a}\left(-\frac{1}{3}\right) \neq 0
$$

and $B_{a}>0$ allow to find $\phi$ as desired also in this case.

If $\mathrm{v}_{1}^{z, a}(0) \neq 0 \neq \mathrm{v}_{2}^{z}, \mathrm{a}(0)$, choose a sequence of continuous functions $\omega_{n}:\left[-\frac{1}{3}, 0\right] \rightarrow \underline{C}$ with $\omega_{n}\left(-\frac{1}{3}\right)=0=\omega_{n}(0)$ for all $n \in \underline{N}$ such that

$$
\begin{aligned}
& \int_{-\frac{1}{3}}^{0} A_{a} \omega_{n}\left(-v_{2}^{z, a}\right) \rightarrow-A_{a}\left(-\frac{1}{3}\right) v_{2}^{z, a}\left(-\frac{1}{3}\right)=0, \\
& \int_{-\frac{1}{3}}^{0} A_{a} \omega_{n} v_{1}^{z, a} \rightarrow A_{a}\left(-\frac{1}{3}\right) v_{1}^{z, a}\left(-\frac{1}{3}\right)=A_{a}\left(-\frac{1}{3}\right)>0
\end{aligned}
$$

as $n \rightarrow \infty$. For $n$ sufficiently large and for $\phi$ with $\phi_{2}=\omega_{n}$ and $\phi_{1}=0=\phi_{3}, \quad\left(I_{z}, a^{\phi}\right)_{2} \neq 0$.

3. Consider $\phi \in \tilde{C}$ and $c$ as in part 2. There is a bounded sequenct of points $\psi^{(n)} \in \tilde{c}$ with $\psi_{1}^{(n)}=0, \psi_{2}^{(n)}=\phi_{2}, \psi_{3}^{(n)}(0)=c_{1}$ and $\psi_{3}^{(n)} \mid\left[-\frac{1}{3},-\frac{1}{n}\right]=0$ for all $n \in \mathbb{N}$. It follows that for $n \rightarrow \infty$,

$$
\mathrm{I}_{z, \mathrm{a}^{\psi}}(\mathrm{n}) \rightarrow \cdot\left(\begin{array}{c}
-\mathrm{c}_{1} \\
0
\end{array}\right)+\mathrm{I}_{z, \mathrm{a}^{\phi}}=\left(\begin{array}{l}
0 \\
\mathrm{c}_{2}
\end{array}\right) \neq 0 \text {. }
$$

REMARK: Lemma 1 and Proposition 1 are the analogue of Lemma 3.1 from [31], The operator $\mathrm{L}_{z, a}$ corresponds to $\mathrm{L}(\lambda)$ in [31]. In [31] we did not explicitly show that $L(\lambda)$ is surjective. Let us mention that a proof of this requires that the function $[0,1] \exists t \rightarrow g^{\prime}(x(t)) \in \underline{R}$ (which corresponds to $A_{a}$ or $B_{a}$ here) has at most finitely many zeros. This property is satisfied for the functions $g$ in sections $4-6$ of [31] but was forgotten as an extra hypothesis in Section 3 of [31].

$$
\begin{aligned}
& \text { For } 0 \neq z \in C \text {, set } \\
& Q_{z, a}^{*}:=\left(\begin{array}{ll}
v_{2}^{z, a}(0) & z-u_{2}^{z, a}(0) \\
1-v_{1}^{z, a}(0) & u_{1}^{z, a}(0)
\end{array}\right) .
\end{aligned}
$$

PROPOSITION 2. The analytic map $z+q_{a}(z)\left(\tilde{W}_{a}-z\right)^{-1}$ from $\underline{\mathrm{C}}-\left(\sigma\left(\mathrm{W}_{\mathrm{a}}\right) \cup\{0\}\right)$. into $\tilde{C}^{\mathrm{C}}$ has a continuous extension $\mathrm{H}_{\mathrm{a}}$ to $\underline{\mathrm{C}}-\{0\}$ with

$$
\left(\begin{array}{c}
\left(\mathrm{H}_{\mathrm{a}}(\mathrm{z})_{\phi}\right)_{3} \\
\left(\mathrm{H}_{\mathrm{a}}(\mathrm{z}) \phi\right)_{2}
\end{array}\right)(t)=\frac{1}{z} S_{z, a}(t)\left(-Q_{z_{r}}^{*} \mathrm{a}_{z, a^{\phi}}\right)
$$

for $t \in\left[-\frac{1}{3}, 0\right], 0 \neq z \in \sigma\left(w_{a}\right), \phi \in \tilde{C}$.

PROOF. 1. FOr $\phi \in \tilde{C}$ and $z \in \underline{C}$ with $0 \neq z \notin \sigma\left(w_{a}\right)$, set $x_{z, \phi}:=$ $\left(\tilde{w}_{a}-z\right)^{-1} \phi$. Then $x_{z, \phi}=\frac{1}{z}\left(\tilde{w}_{a} x_{z, \phi}-\phi\right)$. It remains to show that the map $H^{*}: \underline{C}-\left(\sigma\left(W_{a}\right) \cup\{0\}\right) \rightarrow \tilde{C}^{C} ; H^{*}(z) \phi=q_{a}(z) \tilde{w}_{a} x_{z, \phi}$, has a continuous extension $H^{\prime}$ to all of $\subseteq-\{0\}$.

Consider first the maps $H_{i}^{*}, 1=1,2,3$, from $\underline{C}-(\ldots)$ to the complex Banach space of linear continuous maps $\tilde{\mathrm{C}} \rightarrow \mathrm{C}\left(\left[-\frac{1}{3}, 0\right], \underline{C}\right)$ which are obtained from the application of $J$ to $H^{*}(z) \phi$ :

$\left(H_{1}^{*}(z) \phi, H_{2}^{*}(z) \phi, H_{3}^{*}(z) \phi\right)=J H^{*}(z) \phi \in J \tilde{C}$

for all $z \in \underline{c}-(\ldots), \phi \in \tilde{c}$.

2. Let $z \in \underline{C}, 0 \neq z \notin \sigma\left(W_{a}\right), \phi \in \tilde{C}$. By Corollary $1, q_{a}(z) \neq 0$. We compute $\frac{1}{q_{a}(z)} H_{i}^{*}(z) \phi=\left(\tilde{w}_{a} x_{z, \phi}\right)_{i}$ for $i=1,2,3$ :

and from Lemma 1 ,

$$
\left(\tilde{w}_{\mathrm{a}} \mathrm{x}_{\mathrm{z}, \phi}\right)_{1}=\left(\mathrm{x}_{\mathrm{z}, \phi}\right)_{3}=\frac{1}{z}\left(\left(\tilde{\mathrm{w}}_{\mathrm{a}} \mathrm{x}_{\mathrm{z}, \phi}\right)_{3}-\phi_{3}\right) \text {, }
$$

$$
\left(\begin{array}{l}
\left(w_{a} x_{z, \phi}\right)_{3} \\
\left(w_{a} x_{z, \phi}\right)_{2}
\end{array}\right)=s_{z, a}[c+I(z, a, \phi, \cdot)]
$$

on the interval $\left[-\frac{1}{3}, 0\right]$, where $-Q_{z, a^{c}}=I_{z}, a^{\phi}$, or equivalently, 


\section{$c=\frac{-1}{q_{a}(z)} Q_{z, a^{*}}^{I_{z}, a^{\phi}}$}

3. The maps $0 \neq z+u_{i}^{z, a} \mid\left[-\frac{1}{3}, 0\right]$ and $0 \neq z \rightarrow v_{i}^{z, a} \mid\left[-\frac{1}{3}, 0\right]$,

$i=1,2$, are continuous with respect to uniform convergence on the interval $\left[-\frac{1}{3}, 0\right]$. From the computation above we infer that $\mathrm{H}_{2}^{\frac{\alpha}{2}}$ and $\mathrm{H}_{3}^{*}$, and therefore also the map

$$
H_{1}^{*}: z \rightarrow H_{1}^{*}(z)=\frac{1}{z} H_{3}^{*}(z)-\frac{q_{a}(z)}{z} J_{3},
$$

where, $J_{3} \phi=\phi \mid\left[-\frac{1}{3}, 0\right]$ for $\phi \in \tilde{C}$,

have continuous extensions $H_{i}^{1}$ to $\underline{C}-\{0\}, i=1,2,3$.

For example, if $z \neq 0$, if $\phi \in \tilde{C}$ and $t \in\left[-\frac{1}{3}, 0\right]$ then

$\left(H_{3}^{\prime}(z) \phi\right)(t)$ equals the first component of

$$
-S_{z, a}(t) Q_{z, a^{\phi}}^{*}+q_{a}(z) S_{z, a}(t) I(z, a, \phi, t) .
$$

4. For all $z \in \sigma\left(W_{a}\right)-\{0\}$ and a.ll $\phi \in \tilde{C}$,

$\left(H_{1}^{\prime}(z) \phi, H_{2}^{\prime}(z) \phi, H_{3}^{\prime}(z) \phi\right)=\lim _{\zeta \rightarrow z}\left(H_{1}^{*}(\zeta) \phi, H_{2}^{*}(\zeta) \phi, H_{3}^{*}(\zeta) \phi\right) \in J \tilde{C}$.

Set $H^{\prime}(z) \phi:=J^{-1}\left(H_{1}^{\prime}(z) \phi, H_{2}^{\prime}(z) \phi, H_{3}^{\prime}(z) \phi\right)$, for $0 \neq z \in \subseteq$ and $\phi \in \tilde{C}$.

5. In case $0 \neq z \in \sigma\left(w_{a}\right), q_{a}(z)=0$. This implies the formula for the third and second component of $\mathrm{JH}_{\mathrm{a}}(z) \phi, \phi \in \tilde{\mathrm{C}}$.

COROTIARY 2. Let $0 \neq z \in \sigma\left(W_{a}\right), Q_{z, a} \neq 0$. Then $H_{a}(z) \neq 0 \in \tilde{C}^{C}$. PROOF. $Q_{z, a} \neq 0$ yields $Q_{z, a}^{*} \neq 0$, or $Q_{z, a^{c}}^{*} \neq 0$ for some $c \in \underline{C}^{2}$. By proposition 1, $c=I_{z}, a^{\phi}$ for some $\phi \in \tilde{c}$. Apply the last formula in Proposition 2.

COROLIARY 3. Let $0 \neq z \in \sigma\left(w_{a}\right), Q_{z, a} \neq 0$. Then the geometric eigenspace ker $\left(\tilde{w}_{a}-z\right)$ has dimension 1 , and $m_{a}(z)=j_{a}(z)$.

PROOF. 1. Proof of dim ker $\left(\tilde{w}_{a}-z\right)=1$ : Recall temma 1. Consider the map $u$ which sends $x \in \operatorname{ker}\left(\tilde{w}_{a}-z\right)$ to $\left(\begin{array}{l}u \\ v\end{array}\right)\left(-\frac{1}{3}\right)$ where $J \tilde{w}_{a} x=$ $\left(x_{3}, v, u\right)$ : We have $\mu(\operatorname{ker}(\ldots)) \in\left\{c \in \underline{c}^{2}: Q_{z}, a^{c}=0\right\} . \mu$ is injective (Proof: $u x=0$ and (1) with $\phi=0$ give $u=0=v$, hence $x_{3}=\frac{u}{z}=0$ and $x_{2}=\frac{v}{2}=0, x_{1}=\frac{1}{2} x_{3}=0$, and $\left.x=0,.\right)$.

By $0 \neq z \in \sigma\left(w_{a}\right)$ and $Q_{z, a} \neq 0$,

$1 \leqq \operatorname{dim} \operatorname{ker}\left(\tilde{w}_{\mathrm{a}}-z\right)=\operatorname{dim} \mu(\operatorname{ker}(\ldots)) \leqq \operatorname{dim}\{\ldots\}=1$.

2. We have $m_{a}(z)=d i m$ ker $\left(\tilde{w}_{a}-z\right)^{n}$ where $n$ is the minimum of integers $k \in \underline{\mathbb{N}}$ with $\operatorname{ker}\left(\tilde{\mathrm{w}}_{\mathrm{a}}-z\right)^{\mathrm{k}}=\operatorname{ker}\left(\tilde{\mathrm{w}}_{\mathrm{a}}-z\right)^{\mathrm{k}+1}$.
By part $1, n=\Sigma_{1}^{n}$ dim ker $\left(\tilde{w}_{a}-z\right) \geq \operatorname{dim} \operatorname{ker}\left(\tilde{w}_{a}-z\right)^{n}=m_{a}(z)$.

using also

$\operatorname{dim} \operatorname{ker}\left(\tilde{w}_{a}-z\right)<\operatorname{dim} \operatorname{ker}\left(\tilde{w}_{a}-z\right)^{2}<\ldots<\operatorname{dim} \operatorname{ker}\left(\tilde{w}_{a}-z\right)^{n}$

$$
=m_{a}(z)
$$

(provided $1<2<\ldots<n$ ) we infer $m_{a}(z)=n$.

3. $n$ is equal to the order of the pole of the resolvent of $\tilde{w}_{a}$ at $z$. It follows that $\mathrm{n}=\min \left\{k \in \underline{\mathbb{N}}: \zeta \rightarrow(\zeta-z)^{k}\left(\tilde{\mathrm{W}}_{\mathrm{a}}-\zeta\right)^{-1}\right.$ has a continuous extension to $\left.\left(\underline{C}-\sigma\left(w_{a}\right)\right) \cup\{z\}\right\}$. By definition of $j_{a}(z)=: j$, $q_{a}(\zeta)=(\zeta-z)^{j} h(\zeta)$ for $\zeta$ in a neighborhood of $z$, with $h$ analytic and $h(z) \neq 0$. Use Proposition 2 and Corollary 2 to deduce $j=n$, and $j_{a}(z)=m_{a}(z)$.

PROPOSITION 3. FOr $-1 \neq z \neq 0, Q_{z, a} \neq 0$. PROOF. $Q_{z, a}=0$ implies $u_{2}^{z, a}(0)=z$ and $v_{1}^{z, a}(0)=1$. With $0=$ det $e_{z, a}=q_{a}(z)$, we find $z=-1$. 


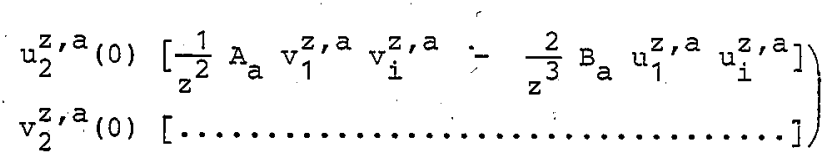

5. ZEROS OF $q_{2}$

PROPOSITION 1, For all a $\in\left(\alpha, a_{2}\right), \lim _{\mathrm{R}} \inf _{\mathrm{z} \rightarrow-\infty} q_{\bar{a}}(z)>0$

PROOF. FOr $\underline{R} \exists z \rightarrow-\infty$, the columns of $S_{z, a}(0)$ converge to the values at $t=0$. of the solutions of $\dot{u}=0=\dot{v}$ with initial values $\left(\begin{array}{l}1 \\ 0\end{array}\right)$,

$\left(\begin{array}{l}0 \\ 1\end{array}\right)$ at $t=-\frac{1}{3}$. It follows that $\frac{1}{z} g_{a}(z) \rightarrow-1$.

From Corollaries 3.1 and $4.1, q_{a}(-1)=0$ for $a<a<a_{2}$. We want to show $m_{a}(-1)=1$ and $-1<\xi_{a}^{*}$ for the other eigenvalue of $w_{a}$ close to -1 (recall the end of section 3$) \cdot m_{a}(-1)=1$ will follow from corollary 4.3 provided we have $Q_{-1, a} \neq 0$ and $q_{a}^{\prime}(-1)^{\prime} \neq 0$. The definition of $Q_{-1, a}$ and the subsequent computation show that

(1) $u_{1}^{-1, a}(0)>0$

is sufficient for both inequalities.

PROPOSITION 2. Let $a \in\left(\alpha_{1}, a_{2}\right)$. Then $v_{2}^{-1, a}(0)=0$ and

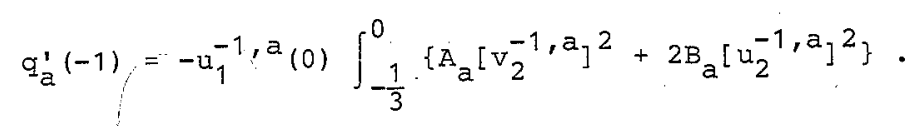

PROOF. 1. FOr $0 \neq z \in \underline{C}$,

(2) $\quad q_{a}^{\prime}(z)=-1+\bar{u}_{2}^{z}(0)+v_{1}^{z, a}(0)+z \cdot \bar{v}_{1}^{z}(0)$

with the solutions $\left(\bar{u}_{i}^{z}, \bar{v}_{i}^{z}\right): \underline{R} \rightarrow \underline{C}^{2}, i=1,2$, of the systems

$$
\begin{aligned}
& \dot{\bar{u}}=\frac{1}{z} A_{a}(t) \bar{v}-\frac{1}{z^{2}} A_{a}(t) v_{i}^{z, a}(t) \\
& \dot{\bar{v}}=\frac{1}{z^{2}} B_{a}(t) \bar{u}-\frac{2}{z^{3}} B_{a}(t) u_{i}^{z, a}(t)
\end{aligned}
$$

with initial conditions $0 \in \underline{C}^{2}$ at $t=-\frac{1}{3}$.

2. Using variations of constants and $\operatorname{det} s_{z, a}(t)=1$ on $\underline{R}$, we get

$$
\begin{aligned}
& \left(\begin{array}{c}
\bar{u}_{i}^{z} \\
\bar{v}_{i}^{z}
\end{array}\right)(0)=s_{z, a}(0) \int_{-\frac{1}{3}}^{j}\left(\begin{array}{cc}
v_{2}^{z, a} & -u_{2}^{z, a} \\
-v_{1}^{z, a} & u_{1}^{z, a}
\end{array}\right)\left(\begin{array}{cc}
-\frac{1}{z^{2}} A_{a} & v_{i}^{z, a} \\
-\frac{2}{z^{3}} B_{a} & u_{i}^{z, a}
\end{array}\right)
\end{aligned}
$$

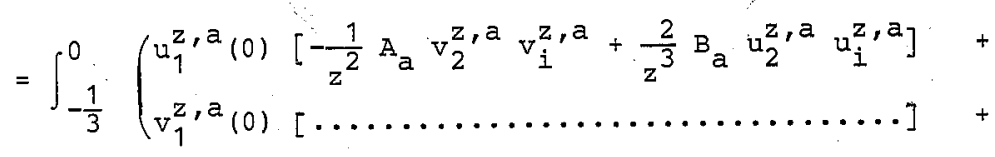

for $i=1,2$. With (2), we obtain

$$
\begin{aligned}
g_{a}^{\prime}(-1)= & -1+\left(-u_{1}^{-1, a}(0) \int_{-\frac{1}{3}}^{0}\left\{A_{a}\left[\dot{v}_{2}^{-1, a^{2}}\right]^{2}+2 B_{a}\left[u_{2}^{-1, a}\right]^{2}\right\}\right. \\
& \left.-\int_{-\frac{1}{3}}^{0}\left\{A_{a} v_{1}^{-1, a} v_{2}^{-1, a}+2 B_{a} u_{1}^{-1, a} u_{2}^{-1, a}\right\}\right)+v_{1}^{-1, a}(0) \\
& +(-1)\left(-\int_{-\frac{1}{3}}^{0}\left\{A_{a} v_{1}^{-1, a} v_{2}^{-1, a}+2 B_{a} u_{1}^{-1, a} u_{2}^{-1, a}\right\}\right)
\end{aligned}
$$

3. Proof of $v_{2}^{-1, a}(0)=0$ and $v_{1}^{-1, a}(0)=1$ : Recall $\left(w_{a}+1\right) \dot{x}_{a, 0}$ $=0$ and $\dot{x}_{a}(0)<0, \dot{x}_{a}\left(\frac{1}{3}\right)=0$. - Lema 4.1 implies that $\left(\begin{array}{l}u \\ v\end{array}\right)$ with $J \tilde{w}_{a} \dot{x}_{a, 0}=(\ldots, v, u)$ is a solution of eq. (4.1) with $z=-1$ and $\phi_{1}=$ $\phi_{2}=\phi_{3}=0$, and we have $u\left(-\frac{1}{3}\right)=\dot{x}_{a}\left(\frac{1}{3}\right)=0, v\left(-\frac{1}{3}\right)=\dot{x}_{a}(0)<0$. Hence

$$
\left(\begin{array}{l}
u \\
v
\end{array}\right)=\dot{x}_{a}(0)\left(\begin{array}{c}
u_{2}^{-1, a} \\
v_{2}^{-1, a}
\end{array}\right) \text {. }
$$

With $v(0)=\dot{x}_{a}\left(-\frac{1}{3}\right)$ and $u(0)=\dot{x}_{a}\left(\frac{2}{3}\right)=-\dot{x}_{a}(0), S_{-1, a}(0)=\left(\begin{array}{cc}* & -1 \\ * & 0\end{array}\right)$ so that $\operatorname{det} s_{-1, a}(0)=1$ yields $v_{1}^{-1, a}(0)=1$.

In order to derive (1) we introduce polar coordinates. The columns of $s_{-1, a}, \alpha<a<a_{2}$, i.e. the solutions of

$$
\dot{u}=-A_{a}(t) v, \ddot{v}=B_{a}(t) u
$$

with initial conditions $\left(\begin{array}{l}1 \\ 0\end{array}\right)$ and $\left(\begin{array}{l}0 \\ 1\end{array}\right)$ at $t=-\frac{1}{3}$, are given by

$$
\left(u_{i}^{-1}, a, v_{i}^{-1}, a\right)=r_{i}^{a}\left(\cos \theta_{i}^{a}, \sin \theta_{i}^{a}\right), i=1,2 \text {, }
$$

with the solutions $\left(r_{i}^{a}, \theta_{i}^{a}\right): \underline{R} \rightarrow \underline{R}^{2}$ of the nonlinear, decoupled system

$$
\begin{aligned}
& \dot{r}=r\left[B_{a}(t)-A_{a}(t)\right] \sin \theta \cdot \cos \theta \\
& \dot{\theta}=A_{a}(t) \sin ^{2} \theta+B_{a}(t) \cos ^{2} \theta=A_{a}(t)+\left[B_{a}(t)-A_{a}(t)\right] \cos ^{2} \theta
\end{aligned}
$$
such that $r_{1}^{a}\left(-\frac{1}{3}\right)=1=r_{2}^{a}\left(-\frac{1}{3}\right), \theta_{1}^{a}\left(-\frac{1}{3}\right)=0, \theta_{2}^{a}\left(-\frac{1}{3}\right)=\frac{\pi}{2}$. Therefore it is enough to show

PROPOSITION 3. There exists $a_{3} \in\left(a, a_{2}\right)$ with

$$
0 \leq \theta_{1}^{a}(0)<\frac{\pi}{2}
$$


for $a<a<a_{3}$.

PROOF. 1. There exists $a_{3} \in\left(\alpha, a_{2}\right)$ such that $e_{2}^{a}(0)=\pi$ for $a$ in $\left(a, a_{3}\right)$. Proof: $v_{2}^{-1, a}(0)=0$ for $a<a<a_{2}$ gives $\theta_{2}^{a}(0) \in \underline{z} \pi$, for such $a$. Observe that for $a+\alpha=\frac{3 \pi}{2}$, the positive coefficients $A_{a}$ and $B_{a}$ in eq. (3) tend to $\frac{3 \pi}{2}$ uniformly with respect to $t \in \underline{R}$. The relations $\theta_{2}^{a}\left(-\frac{1}{3}\right)=\frac{\pi}{2}$ and $0<\dot{\theta}_{2}^{a}$ on $\left[-\frac{1}{3}, 0\right]$ exclude $\pi \neq \theta_{2}^{a}(0) \in \underline{z} \pi$ for a sufficiently close to $\alpha$.

2. Let $a \in\left(a, a_{3}\right)$. Note $0<\dot{\theta}$ for all solutions of eq. (3).

3. Proof of $\theta_{2}^{a}=\frac{3 \pi}{2}-\theta_{2}^{a}\left(-\frac{1}{3}-\cdot\right)$ : set $\theta^{*}:=\frac{3 \pi}{2}-\theta_{2}^{a}\left(-\frac{1}{3}-\cdot\right)$. Then $\theta *\left(-\frac{1}{3}\right)=\frac{\pi}{2}=\theta_{2}^{a}\left(\frac{1}{3}\right)$. Properties of $x_{a}$ give

$$
g_{0}^{\prime}\left(x_{a}\left(\left(-\frac{1}{3}-\cdot\right)-\frac{1}{3}\right)\right)=g_{0}^{\prime}\left(-x_{a}(-\cdot)\right)=g_{0}^{\prime} \circ x_{a} \text {, or }
$$

(4) $\quad A_{a}\left(-\frac{1}{3}-\cdots\right)=B_{a}$ and $B_{a}\left(-\frac{1}{3}-\cdot\right)=A_{a}$.

Hence

$$
\dot{\theta} *=\dot{\theta}_{2}^{a}\left(-\frac{1}{3}-\cdot\right)=A_{a}(\ldots) \sin ^{2} \cdot \theta_{2}^{a}(\ldots)+B_{a}(\ldots) \cos ^{2} \theta_{2}^{a}(\ldots)
$$$$
=B_{a} \cos ^{2} \theta^{*}+A_{a} \sin ^{2} \theta^{*} \text {, }
$$

and by uniqueness of solutions, $\theta_{2}^{a}=\theta^{*}$.

4. In particular, $\theta_{2}^{a}\left(-\frac{1}{6}\right)=\frac{3 \pi}{4}$. with $0<\dot{\theta}_{2}^{a}$, we get

$$
\theta_{2}^{a}\left(\left(-\frac{1}{6}, 0\right)\right) \subset\left(\frac{3 \pi}{4}, \pi\right) \text { and } \theta_{2}^{a}\left(\left(-\frac{1}{3},-\frac{1}{6}\right)\right) \subset\left(\frac{\pi}{2}, \frac{3 \pi}{4}\right) \text {. }
$$

5. We compare the solution $\theta_{0}: \underline{R} \rightarrow \underline{R}$ of eq. (3) with $\theta_{0}\left(-\frac{1}{6}\right)=\frac{\pi}{4}$ with the solution $\theta_{2}^{\mathrm{a}}$.

5.1. On $\left.\left[-\frac{1}{3},-\frac{1}{6}\right)\right), \theta_{0}>\theta_{2}^{a}-\frac{\pi}{2}$. Proof: Clearly $\theta_{0}\left(-\frac{1}{6}\right)=\theta_{2}^{a}\left(-\frac{1}{6}\right)-\frac{\pi}{2}$. Let $t \in\left[-\frac{1}{3},-\frac{1}{6}\right]$ with $0 \leq \theta_{0}$ in $\left[t,-\frac{1}{6}\right]$ be given. On the interval $\left[t,-\frac{1}{6}\right]$

$$
\dot{\theta}_{0}=\left[A_{a}-B_{a}\right] \sin ^{2} \theta_{0}+B_{a} \text {. }
$$

$0<\dot{\theta}_{0}$ and $\theta_{0}\left(-\frac{1}{6}\right)=\frac{\pi}{4}$ give $0 \leqq \theta_{0}<\frac{\pi}{4}$ on $\left[t,-\frac{1}{6}\right]$, so that $\frac{\pi}{2} \leqq \theta_{2}^{a}$ $\leq \frac{3 \pi}{4}$ on $\left[t,-\frac{1}{6}\right]$, and thereby $\sin ^{2} \theta_{0}<\sin ^{2} \theta_{2}^{a}$ on $\left[\cdot t,-\frac{1}{6}\right]$.

Hypothesis (H2) implies that $B_{a}=a g_{0}^{\prime} O, x_{a}$ is strictly increasing

on $\left[-\frac{1}{3}, 0\right]$, with $A_{a}\left(-\frac{1}{6}\right)=B_{a}\left(-\frac{1}{6}\right)$. Using $(4)$, we infer $A_{a}-B_{a}>0$ on $\left[-\frac{1}{3},-\frac{1}{6}\right]$, and therefore $\dot{\theta}_{0}<\dot{\theta}_{2}^{a}$ on $\left[t,-\frac{1}{6}\right]$. We conclude that $0 \leqq \theta_{0}$ and $\dot{\theta}_{0}<\dot{\theta}_{2}^{a}$ on $\left[-\frac{1}{3},-\frac{1}{6}\right]$.
5.2 On $\left(-\frac{1}{6}, 0\right], \theta_{0}<\theta_{2}^{a}-\frac{\pi}{2}$. Proof (as above): we have $\theta_{0}\left(-\frac{1}{6}\right)=$ $\theta_{2}^{a}\left(-\frac{1}{6}\right)-\frac{\pi}{2}$. Let $t \in\left(-\frac{1}{6}, 0\right]$ with $\theta_{0} \leqq \frac{\pi}{2}$ on $\left[-\frac{1}{6}, t\right]$ be given. On $\left(-\frac{1}{6}, t\right]$,

$$
\dot{\theta}_{0}=\left[B_{a}-A_{a}\right] \cos ^{2} \theta_{0}+A_{a} .
$$

The inequalities $\frac{\pi}{4} \leqq \theta_{0} \leqq \frac{\pi}{2}$ and $\frac{3 \pi}{4}<\theta_{2}^{a} \leqq \pi$ yield $\cos ^{2} \theta_{0}<\cos ^{2} \theta_{2}^{a}$ on $\left(-\frac{1}{6}, t\right] .(H 2)$ and (4) give $B_{a}-A_{a}>0$ on $\left(-\frac{1}{6}, 0\right]$. It follows that $\dot{\theta}_{0}<\dot{\theta}_{2}^{a}$ on $\left(-\frac{1}{6}, t\right]^{-}$,

and one concludes that $\theta_{0} \leqq \frac{\pi}{2}$ and $\dot{\theta}_{0}<\dot{\theta}_{2}^{a}$ on $\left(-\frac{1}{6}, 0\right]$.

6. $\theta_{0}\left(-\frac{1}{3}\right)>\theta_{2}^{a}\left(-\frac{1}{3}\right)-\frac{\pi}{2}=0=\theta_{1}^{a}\left(-\frac{1}{3}\right)$ and $\theta_{0}(0)<\frac{\pi}{2}$ now imply $\theta_{1}^{a}(0)<\frac{\pi}{2}$.

REMARK. Condition (H2) guaranteed a supercritical family of bifurcating periodic solutions (i.e., for $a>a$ ), see section 1 . In the proof of Proposition 3 it is used once more, now to the effect that the supercritical solutions $x_{a}$ have minimal instability: There is only one floquet multiplier outside $\underline{s}$, namely the multiplier inherited from instability of the zero solution, which can not be avoided because of continuity.

COROLIARY 1. Let $a \in\left(\alpha, a_{3}\right)$. Then $\mathrm{m}_{a}(-1)=1$ and $-1<\xi_{a}^{*}<-1+\varepsilon$ for the unique eigenvalue $\xi_{a}^{*}$ of $w_{a}$ in $\left(-1+\underline{C}_{\varepsilon}\right) \backslash\{-1\}$. PROOF. 1. $\theta_{1}^{a}(0) \in\left[0, \frac{\pi}{2}\right)$ implies $u_{1}^{-1, a}(0)>0$. Therefore $Q_{-1, a} \neq 0$, and Corollary 4.3 gives $m_{a}(-1)=j_{a}(-1)$. By Propositions 2 and 3 ,

$$
j_{a}(-1)=1 \text {. }
$$

2. Recall Corollary 3.1 : We infer

$$
1=\sum_{-1} \neq z<-1+\varepsilon^{m_{a}(z)=\sum_{-1}} \neq z<-1+\varepsilon j_{a}(z)
$$

(with Proposition 4.3) $q_{a}$ is analytic. By Proposition 1 and $q_{a}^{\prime}(-1)<0$ (Proposition 3),

$$
\sum_{z}<-1 \quad j_{a}(z) \in 2 \underline{z} \text {. }
$$

It follows that $q_{a}$ has no zero on $(-\infty,-1)$, and one zero in $(-1,-1+\varepsilon)$ which must be the unique eigenvalue $\xi_{a}^{*} \neq-1$ of $w_{a}$ in $-1+\underline{c}_{\varepsilon}$. 


\section{HYPERBOLICITY}

We return to the operators $v_{a}$ from section 3 . Let a $\in\left(\bar{\alpha}_{1} \bar{a}_{3}\right)$. Fror $z \in \underline{C}$ and $n \in \underline{N},\left(\tilde{V}_{a}-z^{2}\right)^{n}=\left(\tilde{w}_{a}-z\right)^{n} \circ\left(\tilde{w}_{a}+z\right)^{n}$, and the results about $\sigma\left(\mathrm{w}_{\mathrm{a}}\right)$ imply $1 \in \sigma\left(\mathrm{V}_{\mathrm{a}}\right), 1<\xi_{\mathrm{a}}^{2} \in \sigma\left(\mathrm{V}_{\mathrm{a}}\right)$, and $|z|<1$ whenever $\left\{1, \xi_{a}^{2}\right\} \not z z \in \sigma\left(V_{a}\right)$.

Furthermore, if $M_{a}(z)$ denotes the algebraic multiplicity of $z$ in C. $\{0\}$ considered as an eigenvalue of $\mathrm{V}_{a}$, then $\mathrm{M}_{a}(1)=1$ and $M_{a}\left(\xi_{a}^{2}\right)=1$.

(We might also have employed the Spectral Mapping Theorem to deduce results for $\mathrm{v}_{a}$ from $\mathrm{w}_{a}$ ! !

This means that $x_{a}$ is a hyperbolic periodic solution of eq. $\left(\operatorname{ag}_{0}\right)$ "with one unstable direction".

PROPOSITION 1. There exist $a_{4} \in\left(a_{r} a_{3}\right)$ and a continuous map $a \rightarrow \psi_{a}$ from $\left(\alpha, a_{4}\right)$ into $c$ such that each $\psi_{a}$ is an eigenvector for the eigenvalue $\xi_{a}^{2}$ of $v_{a}$ with $\left|\psi_{a}\right|=1,0<\psi_{a}$, and $\psi_{a}+\psi_{\alpha}$ as $a \psi_{\alpha}$. PROOF. By Corollary 3.1 (iii), $\xi_{a}^{2} \rightarrow \exp \left(\frac{4}{3} \xi_{\alpha}\right)$ as $a+\alpha$. By (3.2), $\mathrm{V}_{a} \rightarrow \mathrm{V}_{\alpha}$ in $\mathrm{C}^{\mathrm{C}}$ as $a+\alpha$. Recall that the function $\psi_{\alpha}, \psi_{\alpha}(t)=$ $\exp \left(\frac{4}{3} \xi_{\alpha} t\right)$ for $t \in[-1,0]$, is an eigenvector of $\nabla_{\alpha}$ for the eigenvalue $\exp \left(\frac{4}{3} \xi_{\alpha}\right)$ (Section 2). Find a disk $\underline{c}_{\delta}, \delta>0$, and $a ! \in\left(\alpha, a_{3}\right)$ with $\left\{\xi_{a}^{2}\right\}=\left(\exp \left(\frac{4}{3} \xi_{\alpha}\right)+c l \underline{c}_{\delta}\right) \cap \sigma\left(V_{a}\right)$ for $\alpha<a<a^{\prime}$. For such $a$, set

$$
\psi_{a}^{\prime}:=-\frac{1}{2 \pi i} \int_{\Gamma}\left(\tilde{V}_{a}-z\right)^{-1} d z\left(\psi_{\alpha}\right)
$$

with $\Gamma(t):=\exp \left(\frac{4}{3} \xi_{\alpha}\right)+\delta \exp (i t)$ for $0 \leqq t \leq 2 \pi ; i . e$. , apply the eigenprojection from $\tilde{c}$ onto the generalized eigenspace of $\xi_{\mathrm{a}}^{2}$ to the fixed vector $\psi_{\alpha}$ (compare $\left.[4,14]\right)$. Then $\psi_{a}^{\prime}+\psi_{\alpha}$ as $a \downarrow \alpha$. Choose $a_{4}$ in $\left(\alpha, a_{3}\right)$ so small that $0<\psi_{a}$ for $\alpha<a<a_{4}$, and set $\psi_{a}:=\frac{1}{\left|\psi_{a}^{1}\right|} \psi_{a}^{\prime}$ for these a.
7. COMPARISON RESUITS

The elementary estimates in this section constitute a link between lo$\mathrm{cal}$ and global behavior of solutions of eq. $\left(\mathrm{ag}_{0}\right)$. They rely on two simple facts: Positive initial conditions produce increasing solutions, (because of positive feedback, $0<\xi g_{0}(\xi)$ for $\left.0<|\xi| \leqq \gamma\right)$, and a difference of two solutions which is initially positive increases also. The last property is due to monotonicity of $g_{0}$ in $[-\gamma, \gamma]$.

PROPOSITION 1. Let $a>0$. Let solutions $\mathrm{x}:[-1, \infty) \rightarrow \underline{\mathrm{R}}, \mathrm{y}:[-1, \infty) \rightarrow \underline{\mathrm{R}}$ of eq. $\left(a g_{0}\right)$ be given. Set $\phi:=x_{0}, \psi:=y_{0}, d:=x-y$.

(i). If $\psi<\phi$ and $|\mathbf{x}| \leqq \gamma,|y| \leqq \gamma$ on $\left[-1, t^{\prime}\right]$ for some $t^{\prime} \geqq 0$ then $0<d$ on $\left(0, t^{\prime}\right]$ and, for $0<t \leqq t^{\prime}+1$, $0<a g_{0}^{\prime}(\gamma) \min _{[-1,0]} d \leqq a g_{j}^{\prime}(\gamma) d(t-1) \leqq \dot{d}(t) \leqq a d(t-1)$.

(ii) If $\psi<\phi,|y|<\frac{\gamma}{4}$ on $[-1, \infty)$ and $|\phi|<\frac{\gamma}{2}$ then there is $t^{*}>0$ with $x\left(t^{*}\right)=\gamma$ and $|x|<\gamma$ on $\left[-1, t^{*}\right)$. If $\phi<\psi,|y|<\frac{\gamma}{4}$ on $[-1, \infty)$ and $|\phi|<\frac{\gamma}{2}$ then there is $t^{*}>0$ with $x\left(t^{*}\right)=-\gamma$ and $|x|<\gamma$ on $\left[-1, t^{*}\right)$.

PROOE. 1. Note $0<d$ on $[-1,0]$. Suppose $d(t) \leqq 0$ for some $t$ in $\left(0, t^{\prime}\right]$. Then $0<d^{\text {on }}\left[-1, t^{*}\right)$ and $d\left(t^{*}\right)=0$ for some $t^{*} \in\left(0, t^{\prime}\right]$. Hence

$0 \geq \frac{1}{a} \dot{d}\left(t^{*}\right)=g_{0}\left(d\left(t^{*}-1\right)+y\left(t^{*}-1\right)\right)-g_{0}\left(y\left(t^{*}-1\right)\right)$

$=\int_{y\left(t^{*}-1\right)}^{a\left(t^{*}-1\right)+y\left(t^{*}-1\right)} g_{0}^{1}(\xi) d \xi$.

with $0<d(t *-1),|x(t *-1)|<\gamma,|y(t *-1)|<\gamma$ :

$0 \geq d(t *-1) \min _{[-\gamma, \gamma]}^{*} g_{0}^{\prime}$,

a contradiction to $0<g_{0}^{\prime}$ on $[-\gamma, \gamma]$. This proves $0<d$ on $\left(0, t^{\prime}\right]$.

Now let $0<t \leq t^{\prime}+1$. Using eq. $\left(a_{0}\right)$ as before, and $g_{0}^{\prime}(\gamma) \leq g_{0}^{\prime}$ $\leqq 1$ on $[-\gamma, \gamma]$, we infer

$a d(t-1) \geqq \dot{d}(t) \geqq a g_{0}^{\prime}(\gamma) d(t-1)>0$. 
With $0<\dot{d}$ on $\left(0, t^{\prime}+1\right], \operatorname{ag}_{0}^{\prime}(\gamma) d(t-1) \geqq \operatorname{ag}_{0}^{\prime}(\gamma) \min _{[-1,0]} d$.

2. We only consider the case $\psi<\phi$. Suppose $|x| \leq \gamma$ on $[-1, \infty)$. By (i), $0<\operatorname{ag}_{0}^{\prime}(\gamma) \min _{[-1,0]} d \leqq \dot{d}$ on $(0, \infty)$, and $d(t) \rightarrow \infty$ as $t \rightarrow \infty$, a contradiction to $|y|<\frac{\gamma}{4}$ on $[-1, \infty)$.

It follows that $\left|x\left(t^{*}\right)\right|=\gamma$ and $|x|<\gamma$ on $\left[-1, t^{*}\right)$ for some $t^{*}>0$. Suppose $x\left(t^{*}\right)=-\gamma$. Then, by (i), $0<\dot{d}$ on $\left(0, t^{*}+1\right)$, and $d(0)<d\left(t^{*}\right)$, or

$$
x(0)-y(0)<-\gamma-y\left(t^{*}\right), x(0)<-\gamma+\frac{\gamma}{2},
$$

a contradiction to $|\phi|<\frac{\gamma}{2}$.

$$
\text { We choose } a_{5} \in\left(\alpha, a_{4}\right) \text { with } \max _{\underline{R}}\left|x_{a}\right|<\frac{\gamma}{4} \text { for } \alpha<a<a_{5} \text {, and for }
$$
each a $\epsilon\left(a, a_{5}\right)$ a number $r_{0}(a)>0$ so small that for every solution $x$ of eq. $\left(a g_{0}\right)$ with $x_{0} \in \phi_{a}^{*}+c_{r_{0}}(a),|x|<\frac{\gamma}{2}$ on $[-1,2]$.

Proposition 1 yields

COROLLARY 1. Let $a \in\left(\alpha, a_{5}\right)$. For every solution $x:[-1, \infty) \rightarrow \underline{R}$ of eq. $\left(a g_{0}\right)$ with $x_{0} \in \phi_{a}^{*}+C_{r_{0}}(a)$ and $x_{0}>\phi_{a}^{*}\left(x_{0}<\phi_{a}^{*}\right)$ there exists $t^{*}=t^{*}\left(x_{0}, a\right)>2$ such that

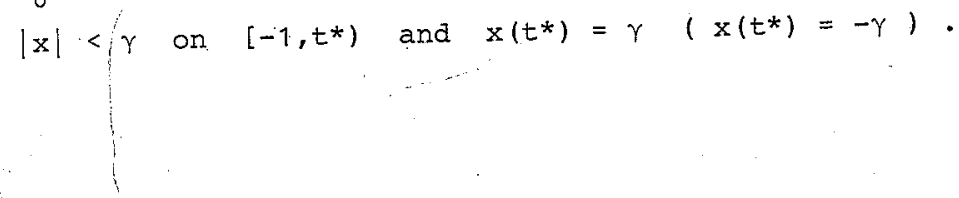

\section{ON HYPERBOLIC FIXED POINTS}

Hypotheses and preliminaries: Let $f: D \rightarrow L-b e$ a $C^{k}-$ map, $k \in \underline{N}$, on an open subset $D$ of a real Banach space $L$ with norm. I I. Suppose $f$ has a hyperbolic fixed point $x^{*}:$ For $A::=D f\left(x^{*}\right)$,

$$
\sigma(A) \cap \underline{s}=\emptyset \text {. }
$$

Then there is a decomposition

$$
\text { (®) } \quad L=L^{u} \oplus L^{S}
$$

into A-invariant closed subspaces such that the induced maps

$L^{\mathrm{U}} \ni \mathrm{x} \rightarrow A \mathrm{Ax} \in \mathrm{L}^{\mathrm{u}}$ and. $\mathrm{L}^{\mathrm{S}} \ni \mathrm{x} \rightarrow A \mathrm{x} \in \mathrm{L}^{\mathrm{S}}$ have spectra outside and inside $\underline{\mathrm{S}}$, respectively. $\mathrm{L}^{\mathrm{u}}$ is called the linear unstable space; $L^{\mathrm{S}}$ is called the linear stable space. The decomposition $(\theta)$ defines projections $p^{\mathrm{u}}$. of $I$ onto $L^{\mathrm{L}}$ and $p^{\mathrm{s}}$ of $L$ onto $I^{\mathrm{s}}$.

occasionally, we shall write $x^{\mathrm{u}}$ for $\mathrm{p}^{\mathrm{u} x}, \mathrm{x}^{\mathrm{s}}$ for $\mathrm{p}^{\mathrm{s} x}$.

The induced map in $I^{u, c}$ is an isomorphism. There exist an equivalent norm $\mid *$, on $L$ and a constant $q \in(0,1)$ with

(u) $|A v|^{*} \geqq q^{-1}|v| *$ on $I^{u}$,

(s) $|A v|^{*} \leqq q|v|^{*}$ on $I^{S}$ :

We say that with respect to $\zeta^{*}$ A is an "expansion-contraction". Existence of ||$^{*}$ follows by arguments from the appendix to Chapter 4.5 in [12]; compare also Theorem 4.19 [12] on hyperbolic isomorphisms.

For $M \subset I$ and $r>0$, $M_{\Upsilon}^{*}:=\left\{X \in M:|\cdot x|^{*}<r\right\}$. 
In the sequel we prepare the proof of a technical statement on attrac-

\section{LOCAI INVARIANT MANIFOIDS}

We restate Theorem 3.1 from [8] on local stabie and unstable manifolds for maps. which are not necessarily diffeomorphisms:

THEOREM 1. There exist positive reals $r(u), \rho(u), r(s), \rho(s)$ and $c^{k}-$ maps $u: L_{r(u)}^{u *} \rightarrow L_{\rho(u)}^{S *}, s: L_{r(s)}^{S *} \rightarrow L_{\rho(s)}^{u *}$ with the following properties.

(i) $u(0)=0, s(0)=0, D u(0)=0, D s(0)=0$

(ii) $\quad U:=x^{*}+\left\{x+u(x): x \in L_{r(u)}^{u *}\right\} \subset D$

$$
S:=x^{*}+\left\{s(x)+x: x \in L_{r(s)}^{S *}\right\} \subset D
$$

(iii) $f \mid S$ is a contraction with respect to ||$^{*} . f(S) \subset S$. For each forward trajectory $\left(x_{n}\right)_{0}^{\infty}$ of $f$ in $x^{*}+L_{p(s)}^{u *}+L_{r(s)}^{S *}, x_{0} \in s$.

(iv). There exists an open neighborhood $D^{\mathrm{L}}$ of $x^{*}$ in $I$ with $f\left(U \cap D^{u}\right)=U$. The restriction $f \mid U \cap D^{u}$ is injective with an inverse $f_{u}^{-1}: U \rightarrow I$ which is a contraction with respect to $\mid l^{*}$. $f_{u}^{-1}$ defines a $c^{k}$-diffeomorphism from the submanifold $v$ onto the submanifold $U \cap D^{\mathrm{u}}$. For every backward trajectory $\left(x_{n}\right)_{-\infty}^{0}$ of $f$ in $x_{i}^{*}+I_{r(u)}^{u *}+I_{\rho(u)}^{S *}, x_{0} \in U$.

For a proof, see $[8,18]$. The "graphs" $U$ and $S$ are called local unstable manifold of $x^{*}$ and local stable manifold of $x^{*}$, respectively. For their tangent spaces, we have

$$
\mathrm{T}_{\mathrm{X}^{*}} \mathrm{U}=\mathrm{L}^{\mathrm{U}}, \mathrm{T}_{\mathrm{X}^{*}} \mathrm{~S}=\mathrm{L}^{\mathrm{S}} \text {. }
$$

An obvious consequence of Theorem 1 which does not involve the new norm ||$^{*}$ any more is

COROLIARY 1. (i) Each $x_{0} \in S$ continues to a unique forward trajectory of $f$ in $S$, with $x_{n} \rightarrow x^{*}$ as $n \rightarrow \infty$.

(ii) Each $x_{0} \in U$ continues to a unique backward trajectory of $f$ in $U$, with $x_{n} \rightarrow x^{*}$ as $n \rightarrow-\infty$ and $x_{n} \in U \cap D^{\mathfrak{u}}$ for all $n \in \underline{N}$.

(iii) For $x \in U \cap D^{U}$, Df $(x)$ induces an isomorphism $T_{X} U \rightarrow T_{f(x)} U$. tion to the local unstable manifold (Proposition 1), and the application of Theorem 5.2 [8] on chaos in Chapter V. Proposition 1 will be used in Chapter III in oräer to link local to global behavior, in the search for heteroclinic solutions.

As in the case of diffeomorphisms in finite dimensions, one can transform $f$ to a map $F$ with hyperbolic fixed point 0 such that $U$ and $S$ become open subsets of $L^{\mathrm{u}}$ and $\mathrm{L}^{\mathrm{s}}$, respectively.

Choose $r>0$ with $r<r(u), r<r(s)$,

$D_{I}:=x^{*}+L_{I}^{U *}+L_{r}^{S *} \subset D$,

Then

$$
|D u(x)| *<\frac{1}{2} \text { on } L_{r}^{u *} \text { and }|D s(x)| *<\frac{1}{2} \text { on } I_{r}^{S *} \text {. }
$$

$$
\begin{aligned}
& U(r):=x^{*}+\left\{x+u(x): x \in I_{r}^{U *}\right\} \subset D_{I}, \\
& S(r):=x^{*}+\left\{s(x)+x: x \in I_{r}^{S *}\right\} \subset D_{I} .
\end{aligned}
$$

For $r^{\prime}>0$ so small that

$$
x^{*}+L_{r,}^{*} \subset D^{u}
$$

and we obtain

$$
\left[f(x)-x^{*}\right]^{u} \in L_{r}^{u *},\left[f(x)-x^{*}\right]^{S} \in I_{r}^{S *} \text { for all } x \in x^{*}+L_{r}^{*}
$$

$f\left(U(r) \cap\left[x^{*}+L_{r}^{*},\right]\right) \subset U(x), f\left(S(r) \cap\left[x^{*}+L_{r}^{*}\right]\right) \subset S(r)$.

(Proof of the first inclusion: For $x \in U(r) \cap\left[x^{*}+L_{r}^{*}\right]^{\top}, x \in U \cap D^{u}$.

Hence $f(x) \in U$, or $f(x)=x^{*}+y+u(y)$ with $y \in L_{r(u)}^{u *} \cdot p^{u} u(y)=0$

and $y=y^{u}$ give $y=\left[f(x)-x^{*}\right]^{u} \in L_{r(u)}^{u+}$, or $f(x) \in U(r)$.)

Now consider the transformation $T_{I}: D_{I} \rightarrow I_{\text {defined by }}$

$$
\begin{aligned}
T_{I}(x) & =x-x^{*}-s\left(\left[x-x^{*}\right]^{s}\right)-u\left(\left[x-x^{*}\right]^{u}\right) \\
& =\left[x-x^{*}\right]^{u}-s\left(\left[x-x^{*}\right]^{s}\right)+\left[x-x^{*}\right]^{s}-u\left(\left[x-x^{*}\right]^{u}\right)
\end{aligned}
$$

for all $x \in D_{I}$. Note

$$
T_{I}\left(x^{*}\right)=0 \text { and } D T_{I}\left(x^{*}\right)=i d \text {. }
$$


3. Proof of $V_{I I} \cap I^{U} \in T_{I I}\left(D_{I I} \cap U\right)$ : For $y \in V_{I I} \cap L^{U}$,

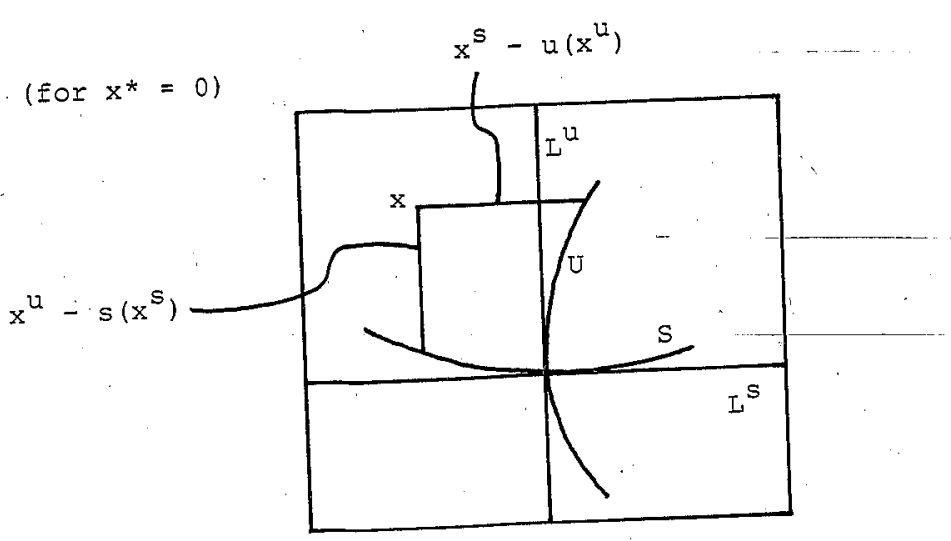

$x:=T_{I I}^{-1}(y) \in D_{I I} \subset D_{I}$. By Corollary 2 (i), $x \in U(r) \subset U$.

4. It follows that ${ }^{T}{ }_{I I}\left(D_{I I} \cap U\right)=V_{I I} \cap I^{u}$, and $T_{I I}$ induces a $C^{k}$ diffeomorphism of the submanifold $D_{I I} \cap U$ onto the submanifold

$V_{I I} \cap I^{U}$. This implies that alI $D_{I I}(x), x \in D_{I I} \cap U$, induce isomorphisms of $T_{x} U$ onto $T_{T_{I I}(x)}\left(V_{I I} \cap L^{u}\right)=T_{T_{I I}}(x) I^{I^{u}}=L^{u}$.

5. The proof for $x \in D_{\text {II }} \cap S$ is analogous.

Note $f \circ \mathbb{T}_{I I}^{-1}(0)=x^{*} \in D_{I I}$. Continuity implies existence of an open neighborhood $V \subset V_{I I}$ of $0 \in I$ such that $f \circ T_{I I}^{-1}(V) \subset D_{I I}$. $D_{V}:=\mathbb{T}_{I I}^{-1}(V)$ is an open neighborhood of $x^{*}$. The transformed map $F: V \ni y \rightarrow T_{I I}^{\prime \prime}\left(F\left(T_{I I}^{-1}(y)\right)\right) \in I$

COROLIARY 2. (i) Let $x \in D_{I}$. Then $x \in U(x)$ is equivalent to $T_{I}(x) \in L^{u}$, and $x \in S(x)$ is equivalent to $T_{I}(x) \in I^{S}$. is $C^{k}$, with $F(0)=0$ and $D F(0)=A=D f\left(x^{*}\right)$.

(ii)

$x \in U(x)$ implies $D T_{I}(x) T_{X} U \in I^{u}$. For $x \in S(x)$,

$D T_{I}(x) T_{X} S \subset I^{S}$.

The Implicit Function Theorem yields open neighborhoods $D_{I I} \in D_{I}$ of $x^{*}$ and $V_{I I}$ of $0 \in I$ such that $T_{I I}:=\left.\mathbb{T}_{I}\right|_{I I}$ maps $D_{I I}$ onto $V_{I I}$ and has a $C^{k}$-inverse $\mathrm{T}_{I I}^{-1}: V_{I I} \rightarrow \mathrm{L}$, with $\mathrm{T}_{I I}, \mathrm{~T}_{I I}^{-1}, \mathrm{DT}_{I I}$ and $\mathrm{DT}_{I I}^{-1}$ aII bounded and $D_{I I} \subset x^{*}+I_{Y}^{*}$.

COROLLARY 3. (i) The maps $D T_{I I}(x), x \in D_{I I}$, are isomorphisms.

(ii) If $x \in D_{I I} \cap U$ then $D_{I I}(x)$ induces an isomorphism from $T_{x} U$ onto $I^{\text {LI }}$.

(i1i) If $x \in D_{I I} \cap S$ then $D T_{I I}(x)$ induces an isomorphism from $T_{x} S$ onto $L^{5}$.

PROOF. 1. Assertion (i) is obvious from the chain rule.

2. Proof of $T_{I I}\left(D_{I I}\right.$ i $\left.U\right) \in V_{I I} \cap I^{U}$ : we have $D_{I I} \cap U \in D_{I} \cap U C^{\prime}\left(x^{*}+L_{r}^{u *}+I^{s}\right) \cap U=U(x)$. Hence $T_{I I}\left(D_{I I} \cap U\right) \subset \mathbb{T}_{I}\left(D_{I} \cap U(I)\right) \subset I^{u}$, by corollary 2 (i). $T_{I I}\left(D_{I I} \cap U\right) \subset V_{I I}$ is clear.

COROLIARY 4. (i) FOr all $x \in D_{V}, f(x)=T_{I I}^{-1}\left(F\left(T_{I I}(x)\right)\right)$.

(ii) $\quad F\left(V \cap L^{u}\right) \subset L^{u}, F\left(V \cap L^{S}\right) \subset L^{S}$. The remainder

$R: V \ni y \rightarrow F(y)-A y \in L$

is $C^{k}$ with $R(0)=0, D R(0)=0$ and

$R\left(V \cap L^{u}\right)=L^{u}, R\left(V \cap L^{S}\right)<L^{S}$.

(iii) For $y \in \vee \cap I^{\mathrm{U}}, \mathrm{p}^{\mathrm{S}} \mathrm{DR}(\mathrm{y}) \mathrm{p}^{\mathrm{u}}=0$.

For $y \in V \cap \mathrm{L}^{\mathrm{S}}, \mathrm{p}^{\mathrm{u}} \mathrm{DR}(\mathrm{y}) \mathrm{p}^{\mathrm{S}}=0$.

PROOF. 1. $x \in P_{V}$ gives $T_{I I}(x) \in V$, and

$$
f(x)=\mathbb{T}_{I I}^{-1}\left(T_{I I}(f(x))\right) \in f\left(T_{I I}^{-1}(V)\right) \subset D_{I I}=T_{I I}^{-1}\left(V_{I I}\right) .
$$

Therefore

$$
\begin{aligned}
f(x) & =T_{I I}^{-1}\left(T_{I I}(f(x))\right)=T_{I I}^{-1}\left(T_{I I}\left(f\left(T_{I I}^{-1}\left(T_{I I}(x)\right)\right)\right)\right) \\
& \left.=T_{I I}^{-1}\left(F\left(T_{I I}(x)\right)\right)\right) .
\end{aligned}
$$

2. For $y \in V \cap L^{U}, T_{I I}^{-1}(y) \in D_{V} \in D_{I I} \in D_{I}$ and $y=T_{I I}\left(T_{I I}^{-1}(y)\right) \epsilon$ $L^{\text {L }}$. Corollary 2 (i) yields $T_{I I}^{-1}(y) \in U(r)$. With $T_{I I}^{-1}(y) \in D_{I I} \subset x^{*}+I_{r}^{*}+, I\left(T_{I I}^{-1}(y)\right) \in U(x) \cdot B_{Y} f\left(T_{I I}^{-1}(y)\right) \in D_{I I} \subset D_{I}$ and by corollary 2 (i), $F(y)=\mathbb{T}_{I I}\left(f\left(T_{I I}^{-1}(y)\right)\right) \in I^{\mathrm{u}}$. This proves $F\left(V \cap L^{\mathrm{u}}\right) c$ 
tion (ii) are obvious.

3. Proof of $p^{u} D R(y) p^{s}=0$ for $y \in V \cap I^{S}: R\left(V \cap I^{S}\right) \subset L^{S}$ gives $D R(Y) L^{S}=D R(Y) T_{Y}\left(V \cap L^{S}\right) \subset T_{R(Y)} L^{S}=L^{S}$, or $p^{L} D R(Y) p^{S}=0$.

For the proof of Proposition 1 below we need expansion-contraction properties of the nonlinear map $F$. Choose $c=c_{F}>0$ with

$$
q+c<1<q^{-1}-c
$$

and $r(F)>0$ so small that

$$
V_{F}:=L_{r(F)}^{u *}+I_{r(F)}^{S *} \subset V
$$

with

$$
c>\left|p^{u} D R(y)\right|^{*}+\left|p^{S} D R(y)\right|^{*}+|D R(y)| * \text { on } V_{F} \text {. }
$$

COROLIARY 5. FOY $Y \in V_{F}$,

$$
\begin{aligned}
& \left|p^{u} R(y)\right|^{*} \leqq c\left|y^{u}\right|^{*}, \\
& \left(q^{-1}-c\right)\left|y^{u}\right|^{*} \leq\left|p^{u} F(y)\right|^{*} \leqq\left(|A|^{*}+c\right)\left|y^{u}\right|^{*}, \\
& \left|p^{s} F(y)\right|^{*} \leq(q+c)\left|y^{s}\right|^{*} .
\end{aligned}
$$

PROOF. FOr $y \in V_{F}, y^{S} \dot{\epsilon} L_{r(F)}^{S *}=V_{F} \cap L^{S}$. By Corollary 4 (ii), $p^{u} R(Y)=$ $p^{u} R(y)-p^{u} R\left(y^{s}\right)$. The straight line from $y^{s}$ to $y$ is contained in $V_{F}$ so that the Mean value Theorem implies

$$
\left|p^{u} R\left(y^{\prime}\right)\right|^{*} \leq c\left|y-y^{s}\right|^{*}=c\left|y^{u}\right|^{*} \text {. }
$$

Using $(\mathrm{u})$ and $\mathrm{p}^{\mathrm{u}} F(\mathrm{y})=A \mathrm{Y}^{\mathrm{u}}+\mathrm{p}^{\mathrm{u}} \mathrm{R}(\mathrm{Y})$ one obtains the estimate of $\mathrm{p}^{\mathrm{u}} \mathrm{F}(\mathrm{y})$. The estimate of $\mathrm{p}^{\mathrm{s}} \mathrm{F}(\mathrm{y})$ is proved analogously.

PROPOSITION 1. Assume $\operatorname{dim} L^{\mathrm{u}}=1, \mathrm{~L}^{\mathrm{u}}=\underline{R} \lambda$ and $\mathrm{A} \lambda=\xi \lambda$ with $\xi>1$, and $[-\rho, p] \lambda \subset \mathrm{L}_{\mathrm{r}(\mathrm{u})}^{\mathrm{u}}$ with $\rho>0$.

There exists $0^{\prime} \in(0,0)$ such that for every open neighborhood $\Delta$

$$
\text { of } \left.x^{*}+\left\{x+u(x): x \in\left[\rho^{1}, p\right] \lambda\right\} \text { (of } \ldots\left[-p,-p^{2}\right] \lambda\right\} \text { ) }
$$

there is $I(\Delta)>0$ with the following properties.

(i) $p^{s} L_{r(\Delta)}^{*} \subset L_{r(s)}^{S *}$

(ii) $\quad x^{*}+I_{r(\Delta)}^{*} \subset D$

(iii) For every $x \in x^{*}+L_{r}^{*}(\Delta)$ with

$$
\left[\dot{x}-x^{*}\right]^{u}-s\left(\left[x-x^{*}\right]^{s}\right) \in(0, \infty) \lambda \quad(\ldots \in \in(-\infty, 0) \lambda)
$$

there exist points $x_{0}=x, x_{1}, \ldots, x_{j} ; j=j(x) \in \underline{N} ;$ in $D$ 


\section{PROOF OF PROPOSITION 1.1}

We give a proof for the statement not in brackets.

1. Consider $\rho>0$ with $[-\rho, \rho] \lambda \subset I_{r(u)}^{\text {ut }}$. Set $D_{F}:=\mathbb{T}_{I I}^{-1}\left(V_{F}\right)$ and $T_{F}:=T_{I I} \mid D_{F}$. Choose $\rho " \in(0, \rho)$ with

$$
x^{*}+\left\{x+u(x): x \in\left[0, \rho^{n}\right] \lambda\right\} \subset D_{F} \text {. }
$$

Proof that there exists $\rho_{1}>0$ with $\left[0, p_{1}\right] \lambda \subset \mathbb{T}_{F}\left(x^{*}+\{\ldots\}\right)$ :

The estimates of $D u(x)$ and $D s(x)$ on $L_{r}^{u *}$ and $L_{x}^{S *}$, respectively, in Section 1 and the Mean Value Theorem imply

$$
|u(x)|^{*} \leqq \frac{1}{2}|x|^{*}<\frac{r}{2} \text { on } L_{r}^{u *},|s(x)|^{*} \leqq \frac{1}{2}|x|^{*}<\frac{r}{2} \text { on } I_{r}^{S *} \text {. }
$$

Choose $\rho_{1}$ so small that for all $y \in\left[0, \rho_{1}\right] \dot{\lambda} \subset L^{u}$,

$$
y \in V_{F} \text { and }\left|\left[T_{F}^{-1}(y)-x^{*}\right]^{u}\right| * \leq \rho^{\prime}|\lambda|^{*} \text {. }
$$

For such $y, z:=\mathbb{T}_{F}^{-1}(y)$ lies in $D_{F} \in D_{I I} \in D_{I} \in D$. Corollary 1.2 . (i) gives $z \in U(r)$, or $z=x^{*}+x+u(x)$ with $x \in L_{r}^{u *}=(-r, r) \frac{1}{|\lambda|^{*}} \lambda$.

We have $x \in\left[-\rho^{n}, \rho^{\prime \prime}\right] \lambda$ since $|x|^{*}=\left|\left[z-x^{*}\right]^{u}\right|^{*} \leq \rho^{n}|\lambda|^{*}$. It remains to show that $x \in\left[0, \rho^{\prime \prime}\right] \lambda . x=\underline{\beta} \lambda$ with $-\rho " \leqq \underline{\beta} \leqq \rho "$ and $y=\underline{\alpha} \lambda$ with $0 \leqq \underline{\alpha} \leqq p_{1}$ imply

$$
\underline{\alpha} \lambda=y=\mathbb{T}_{F}(z)=\mathbb{T}_{F}\left(x^{*}+\underline{\beta} \lambda+u(\underline{\beta} \lambda)\right)=\underline{\beta} \lambda-s(u(\underline{\beta} \lambda)),
$$

see the definition of $T_{I}$. We have $s(\ldots)=\underline{\gamma} \lambda$ with $\underline{\alpha} \lambda=(\underline{\beta}-\underline{\gamma}) \lambda$.

By $\underline{\beta} \lambda=x \in L_{r}^{u *},|u(\underline{\beta} \lambda)|^{*} \leqq \frac{1}{2}|\underline{\beta} \lambda|^{*}$. Therefore $u(\underline{\beta} \lambda) \in \mathrm{L}_{r}^{\mathrm{S*}}$, and

$$
|\underline{\gamma} \lambda|^{*}=|s(u(\underline{\beta} \lambda))|^{*} \leqq \frac{1}{2}|u(\underline{\beta} \lambda)| * \leqq \frac{1}{4}|\underline{\beta} \lambda| * \text {, }
$$

or $|\underline{Y}| \leqq \frac{1}{4}|\underline{B}|$. This yields $\underline{B}-\underline{Y}=0$ and $\underline{\beta}=0$ in case $\underline{\alpha}=0$, and $\underline{\beta}-\underline{Y}>0$ and $\underline{B}>0$ in case $\underline{\alpha}>0$.

2. The next aim is a variant of Proposition 1.1 for the map $F$. We show that there exists $\rho_{j} \in\left(0, \rho_{1}\right)$ such that for every open neighborhood of $\left[\rho_{1}, \rho_{1}\right] \lambda$ there is $r(\Delta, F)>0$ with the following properties:

$L_{\Upsilon}^{*}(\Delta, F) \subset V_{F}$,

and for every $y \in L_{r(\Delta, F)}^{\star}\left(L^{S}\right.$, with $y^{\mathbb{U}} \in(0, \infty) \lambda$ there exist points $y_{0}=y, y_{1}, \ldots, y_{j} ; j=j(y) \in \underline{N} ;$ in $V_{F}$ with
$\mathrm{y}_{\mathrm{k}+1}=\mathrm{F}\left(\mathrm{y}_{\mathrm{k}}\right)$ for $\mathrm{k}=0, \ldots, j-1$ and $\mathrm{y}_{\mathrm{j}} \in \Delta$.

2.1. Choose $\rho_{1}^{n} \in\left(0, \rho_{1}\right)$ with

(1) $\rho . \eta 1|\lambda|^{*}<r_{F}$

and $\rho_{j}^{\prime} \in\left(0, \rho_{1}^{\prime \prime}\right)$. with

(2) $\quad(|A| *+C) \rho_{1}^{i}<\rho_{1}^{n}$.

Let an open neighborhood $\Delta$ of $\left[p_{1}, p_{1}\right] \lambda$ be given. By compactness, there exists $\varepsilon \in\left(0, r_{F}\right)$ with

(3) $\left[\rho_{1}^{\prime}, \rho_{1}\right] \lambda+L_{\varepsilon}^{S *} \subset \Delta$.

Choose $r(\Delta, F)>0$ so small that for all $y \in I_{x(\Delta, F)}^{*}$,

(4) $\quad\left|\mathrm{y}^{\mathrm{u}}\right|^{*}<\rho_{1}^{\prime}|\lambda| *$,

(5) $\left|\mathrm{y}^{\mathrm{S}}\right|^{*}<\varepsilon<\mathrm{r}_{\mathrm{F}}$.

It follows that $I_{r(\Delta, F)}^{*} \subset V_{F}$.

Let $y \in I_{r(\Delta, F)}^{*} \backslash L^{S}$. Then $y^{u} \neq 0$. Assume $y^{u} \in(0, \infty) \lambda$.

2.2. There is no forward trajectory $\left(\mathrm{y}_{\mathrm{k}}\right)_{0}^{\infty}$ of $\mathrm{F}$ in $\mathrm{V}_{\mathrm{F}}$ with $\mathrm{y}_{0}=\mathrm{y}$. Proof: Otherwise, Corollary 1.5 yields

$\left|y_{k}^{u}\right| * \geqq\left(q^{-1}-c\right)^{k}\left|y^{u}\right| *>0$ for all $k, \in \underline{N}$,

a contradiction to $\mathrm{y}_{\mathrm{k}} \in \mathrm{V}_{\mathrm{F}}$ for all $\mathrm{k} \in \underline{\mathrm{N}}$.

2.3. It, follows that there exist points $y_{0}=y, y_{1}, \ldots, y_{k-1}$ in $V_{F}$ and $y_{k} \in L \backslash V_{F}$ with $y_{K+1}=F\left(y_{K}\right)$ for $k=0, \ldots, k-1$. By corollary 1.5 and by (5),

$$
\begin{aligned}
& 0<\left(q^{-1}-c\right)^{k}\left|y^{u}\right|^{*} \leq\left|y_{K}^{u}\right|^{*}, \\
& \text { (6) }\left|y_{K}^{s}\right|^{*} \leq\left|y^{s}\right| *<\varepsilon<r_{F} \text { for } k=0, \ldots, k .
\end{aligned}
$$

We have

$$
\left|y_{k}^{u}\right|^{*}>\rho_{1}^{\prime}|\lambda| *
$$

since otherwise $\left|y_{k}^{u}\right|^{*} \leqq \rho_{1}|\lambda|^{*}<x_{F}$ so that $y_{k} \in v_{F}$ (with (6)), a contradiction.

2.4. Let $j$ denote the smallest integer in $\{0, \ldots, k\}$ with $\rho_{1}^{\prime}|\lambda| *<\left|y_{j}^{\mathrm{u}}\right| *$.

By (4), $1 \leqq j \leq k$. Corollary 1.5 and (2) give

$$
\left|\cdot y_{j}^{u}\right|^{*}=\left|p^{u} F\left(y_{j-1}\right)\right|^{*} \leq\left(|A|^{*}+c\right)\left|y_{j-1}^{u}\right|^{*} \leq\left(|A|^{*}+c\right) \rho_{1}^{\prime}|\lambda| *
$$$$
<\rho_{1}^{\prime \prime}|\lambda|^{*} \text {. }
$$

We observe $y_{j} \in V_{F}$ (from (1) and (6)) and 
$\rho_{1}^{\prime}|\lambda| *<\left|y_{j}^{\mathrm{u}}\right| *<\rho_{1}^{\mathrm{n}}|\lambda| *<\rho_{1}|\lambda| *$,

$$
\left|y_{j}^{S}\right| *<\varepsilon \quad(\sec (6)) \text {. }
$$

In view of (3) and (7) it remains to show that $y_{j}^{u}$ is a positive multiple of $\lambda$. The latter is a consequence of $y_{0}^{u}=y^{\mathfrak{u}} \in(0, \infty) \lambda$, of

$$
y_{K+1}^{u}=A y_{K}^{u}+p^{u} \cdot R\left(y_{k}\right)=\xi y_{K}^{u}+p^{u} R\left(Y_{K}\right)
$$

and

$$
\left.\left|p^{\mathrm{u}} \cdot R\left(\mathrm{y}_{K}\right)\right|^{*} \leq \mathrm{c} \mid \mathrm{Y}_{\mathrm{K}}^{\mathrm{u}}\right]^{*} \quad(\text { Corollary 1.5) }
$$

for $k=0, \ldots, j-1$, and of $c<1<\varepsilon$.

3. We show that there exists $\rho^{\prime} \in\left(0,0^{\prime \prime}\right)$ with

$T_{F}\left(x^{*}+\left\{x+u(x): x \in\left[p^{\prime}, p^{n}\right] \lambda\right\}\right) \supset\left[\rho_{1}, p_{1}\right] \lambda:$

For all $\mathrm{y} \in\left[\rho_{1}, \rho_{1}\right] \lambda, \mathrm{y} \neq 0$ and $\mathrm{T}_{\mathrm{F}}^{-1}(\mathrm{y}) \in \mathrm{U}$; in particular, $\mathrm{T}_{\mathrm{F}}^{-1}(\mathrm{y}) \epsilon$ $U-\left\{x^{*}\right\}$, and thereby $0 \neq\left[T_{F}^{-1}(y)-x^{*}\right]^{u}$. Now compactness implies that there exists $\rho^{\prime} \in\left(0, \rho^{\prime \prime}\right)$ with

(8) $\left|\left[\mathrm{T}_{\mathrm{F}}^{-1}(\mathrm{y})-\mathrm{x}^{*}\right]^{\mathrm{u}}\right|^{*} \geqq \rho^{\prime}|\lambda|^{*}$ for all $\mathrm{y} \in\left[\rho_{1}, \rho_{1}\right] \lambda$.

For such $y, T_{F}^{-1}(y)=x^{*}+x+u(x)$ with $x \in\left[0, p^{\prime \prime}\right] \lambda$, see part 1 . With (8),

$$
|x|^{*}=\left|\left[T_{F}^{-1}(y)-x^{*}\right]^{u}\right|^{*} \geq \rho^{\prime}|\lambda|^{*},
$$

or $x \in\left[\rho^{\prime}, \rho^{\prime \prime}\right] \lambda$.

4. Let $\Delta$ be an open neighborhood of $x^{*}+\left\{x+u(x): x \in\left[\rho^{\prime}, \rho\right] \lambda\right\}=$ $x^{*}+\left\{x+u(x): x \in\left[\rho^{\prime}, \rho^{\prime \prime}\right] \lambda\right\}$ : By the choice of $\rho^{\prime \prime}$,

$$
x^{*}+\left\{x+u(x): x \in\left[\rho^{\prime}, \rho^{\prime \prime}\right] \lambda\right\} \subset D_{F}^{\prime},
$$

and $\Delta^{\prime}:=\mathbb{T}_{F}\left(\Delta \cap D_{F}\right)$ is an open neighborhood of $\left[\rho_{1}^{\prime}, p_{1}\right] \lambda$, see part 3 . Consider $r\left(\Delta^{\prime}, F\right)$ according to part $2 ; I_{x\left(\Delta^{\prime}, F\right)}^{*}=V_{F}$. Choose $x(\Delta)>0$ so small that

$$
x^{*}+L_{r(\Delta)}^{*} \subset D_{F}, T_{F}\left(x^{*}+L_{r(\Delta)}^{*}\right) \subset L_{r\left(\Delta^{\prime}, F\right)}^{*}, p^{S} I_{r(\Delta)}^{*} \subset I_{r(s)}^{S *} \cdot
$$

Consider $x \in x^{*}+L_{r}^{*}(\Delta)$ with $\left[x-x^{*}\right]^{u}-s\left(\left[x-x^{*}\right]^{s}\right) \in(0, \infty) \lambda$. Then $y:=T_{F}(x)^{\prime} \in I_{r}^{*}\left(\Delta^{\prime}, F\right)$. The definition of $T_{I}$ shows that

$$
y^{u}=\left[x-x^{*}\right]^{u}-s\left(\left[x-x^{*}\right]^{5}\right) \in(0, \infty) \lambda \text {. }
$$

The result of part 2 guarantees the existence of points $y_{0}=y, y_{1}, \cdots$ $\ldots, y_{j} ; j \in \underline{N}$; in $V_{F}$ with $y_{k+1}=F\left(y_{k}\right)$ for $k=0, \ldots, j-1$ and $y_{j} \in \Delta^{\prime}$.

Set $x_{k}:=T_{F}^{-1}\left(y_{k}\right)$, for $k=0, \ldots, j$. Then $x_{0}=x, x_{k} \in D_{F} \in D$ for

$$
x_{k+1}=T_{F}^{-1}\left(y_{k+1}\right)=T_{I I}^{-1}\left(F\left(y_{k}\right)\right)=T_{I I}^{-1}\left(F\left(T_{I I}\left(x_{k}\right)\right)\right)=f\left(x_{k}\right)
$$


III. POINCARE MAPS AND SOLUTIONS CLOSE TO $\mathrm{x}_{\mathrm{a}}$

Sumary. The initial values $\phi_{a}^{*}$ of the periodic solutions $x_{a}$ become hyperbolic fixed points of poincare'maps $P_{a}$ on open subsets $D_{a}$ of a hyperplane $\mathrm{H} \subset \mathrm{C}$. The linear unstable spaces are one-dimensional, normalized eigenvectors $\lambda_{a} \in H$ converge as $a+\alpha$ (Corollary 1.1). This permits to describe the position of segments $y_{2}$ of solutions starting in the local unstable manifold of $\phi_{a}^{*}$, with respect to $x_{a, 2}$ in terms of elementary inequalities for $a-\alpha>0$ sufficiently small. Corollary 1.2 contains preparations for this.

The parameter $a$ is then fixed, and dropped. We continue with

$$
g_{1}:=a q_{0} \cdot
$$

Section 2 introduces local stable and unstable manifolds $S$ and $U$ for $\phi^{*}$. Proposition 2.1 is the comparison result for solutions $y$ with $y_{0}$ in $U-S, E$ prepared by Corollary 1.2 .

Section 3 contains the strategy for the search of sine-like nonlinearities with heteroclinic solutions $h$ from $x$ (with $h_{0} \in U \backslash s$ ) to. another periodic solution, and preparations for this search.

In Section 4, a transversality property is explained and characterized.

\section{POINCARE MAPS}

Let $a<a<a_{5}$, and let $x_{a 0}$ denote the semiflow $\underline{R}^{+} \times c \rightarrow c$ given by the solutions $x:[-1, \infty) \rightarrow \underline{R}$ of eq. $\left(\mathrm{ag}_{0}\right)$. The restriction of $x_{a 0}$ to $(1, \infty) \times C$ is $C^{1}$. Consider the hyperplane

$$
\mathrm{H}:=\{\phi \in \mathrm{C}: \phi(0)=0\}=\text { ker ev }
$$

where ev:C $\ni \phi \rightarrow \phi(0) \in \underline{R}$. Note $\phi_{a}^{*}=x_{a, 0} \in \mathrm{H}$.

We have $\operatorname{ev}\left(x_{a 0}\left(\frac{4}{3}, \phi_{a}^{*}\right)\right)=0 . \dot{x}_{a}\left(\frac{4}{3}\right)=\dot{x}_{a}(0)<0$ implies that the partial derivative of

$$
(t, \phi) \rightarrow e v\left(X_{a 0}(t, \phi)\right)=x(t),
$$

with the solution $x:[-1, \infty)+\underline{R}$ of eq. $\left(a_{0}\right)$ starting at $x_{0}=\phi$, with respect to $t$ at $(t, \phi)=\left(\frac{4}{3}, \phi_{a}^{*}\right)$ is different from 0 . An application of the Implicit Function Theorem yields a number $r_{1}(a) \in\left(0, r_{0}(a)\right)$ and a $C^{1}$-map

$$
\tau_{a}: \phi_{a}^{*}+C_{r_{\eta}(a)} \rightarrow(1,2)
$$

with $\tau_{a}\left(\phi_{a}^{*}\right)=\frac{4}{3}$, and for all $\phi \in \phi_{a}^{*}+C_{r_{1}(a)}$,

$$
e v\left(x_{a O}\left(\tau_{a}(\phi), \phi\right)\right)=0 \text {. }
$$

or equivalently,

$$
x_{a 0}\left(\tau_{a}(\phi), \phi\right) \in H,
$$

and finally

$$
\dot{x}\left(\tau_{a}(\phi)\right)<0
$$

with the solution $x:[-1, \infty) \rightarrow \underline{R}$ of eq. $\left(a g_{0}\right)$ starting at $x_{0}=\phi$. We set

$$
N_{a}:=\phi_{a}^{*}+C_{r_{1}(a)} \text {. }
$$

The map $\underline{P}_{a}: N_{a} \ni \phi+\tilde{x}_{a 0}\left(\tau_{a}(\phi), \phi\right) \in H$ is $C^{1}$. Let $i_{H}$ denote the in-

clusion $H+C$. Set $D_{a}:=\phi_{a}^{*}+H_{r_{1}}(a)_{l}=i_{H}^{-1}\left(N_{a}\right)$. The poincare map

$$
P_{a}: D_{a} \ni \phi \rightarrow \underline{P}_{a}\left(i_{H}(\phi)\right) \in H
$$

is $C^{1}$ and has the fixed point $\phi_{a}^{*}$. 
$\mathrm{P}$ is also compact so that there is no continuous inverse in the infinite-dimensional space $\mathrm{H}$.

Note the formula

(1) $\quad \mathrm{P}_{\mathrm{a}}^{j}(\phi)=\mathrm{x}_{\mathrm{a} 0}\left(\sum_{\mathrm{k}}^{j-1} \tau_{\mathrm{a}}\left(\mathrm{P}_{\mathrm{a}}^{\mathrm{k}}(\phi), \phi\right)\right.$

for all $\phi$ in the domain of the iterate. $P_{a}^{j}, j \in \underline{N}$.

The inequality. $\dot{x}\left(\tau_{a}(\phi)\right)<0$, for the solution $x:[-1, \infty) \rightarrow \underline{R}$ of

eq. $\left(\mathrm{ag}_{0}\right)$ with $\mathrm{x}_{0}=\phi \in \mathrm{D}_{\mathrm{a}}$ implies $\left.\dot{\mathrm{x}}_{\tau_{\mathrm{a}}(\phi)}:=\dot{\mathrm{x}}\left(\tau_{\mathrm{a}}(\phi)+\cdot\right)\right\rfloor[-1,0] \notin \mathrm{H}$ or

$$
C=H \oplus \underline{R} \dot{x}_{T a}(\phi)
$$

so that there is a projection $P_{a, \phi}$ of $C$ onto $H$, parallel to the tan gent vector $D_{1} X_{a 0}\left(\tau_{a}(\phi), \phi\right) 1=\dot{x}_{\tau_{a}}(\phi)$ of the trajectory

$$
\underline{R}^{+} \ni t \rightarrow x_{a 0}(t, \phi) \in C
$$

at $t=\tau_{a}(\phi)$. One computes

$$
p_{a, \phi} \psi=\psi-\frac{\psi(0)}{\dot{x}\left(\tau_{a}(\phi)\right)} \dot{x}_{\tau_{a}(\phi)} \text { for all } \psi: \in c \text { : }
$$

As in finite dimensions,

$$
D P_{a}(\phi)=\dot{p}_{a, \phi} \circ D_{2} x_{a 0}\left(\tau_{a}(\phi), \phi\right) \circ i_{H} \text { for all } \phi \in D_{a} \text {. }
$$

More generally, if $\phi$ is in the domain of $P_{a}^{j}, 2 \leq j \in \mathbb{N}$, and if

$\phi_{0}=\phi, \phi_{k+1}=P_{a}\left(\phi_{k}\right), t_{0}=0, t_{k+1}=t_{k}+\tau_{a}\left(\phi_{k}\right)$ for $k=1, \cdots$, $j-1$ then

$$
D P_{a}^{j}(\phi)=P_{a, \phi_{j-1}} \circ D_{2} X_{a 0}\left(t_{j}, \phi\right) \circ i_{H} \cdot
$$

In particular,

$$
\operatorname{DP}_{a}\left(\phi_{a}^{*}\right)=p_{a, \phi_{a}^{*}} \circ V_{a} \circ i_{H} \cdot
$$

From the last formula one obtains (compare [31])

$$
\sigma\left(D P_{a}\left(\phi_{a}^{*}\right)\right)=\sigma\left(V_{a}\right)-\{1\}, \sigma\left(D P\left(\phi_{a}^{*}\right)\right) \cap \underline{s}=\emptyset .
$$

That is, $\phi_{a}^{*}$ is a hyperbolic fixed point of $P_{a}$. The only point in $\sigma\left(D P_{a}\left(\phi_{a}^{*}\right)\right.$. outside $\underline{S}$ is $\xi_{a}^{2}$, an algebraically simple eigenvalue with eigenvector

$$
\lambda_{a}:=p_{a, \phi} \psi_{a} \psi_{a} \in \mathrm{H}^{\circ} .
$$

Define $\zeta \in \mathrm{H}$ by $\zeta(t):=\exp \left(\frac{4}{3} \xi_{\alpha} t\right)-\cos \left(\frac{3}{2} \pi t\right)$. for $-1 \leqq t \leqq 0$.

COROLIARY 1. $\lim _{a \downarrow \alpha} \lambda_{a}=\zeta$
PROOF. FOr $\alpha<a<a_{5}$ and $t \in \underline{R}$, set $\tilde{u}_{a}(t):=\frac{1}{x_{a}(0)} \dot{x}_{a}(t)$ and

$\tilde{v}_{a}(t):=\tilde{u}_{a}(t-1)$. Then $\left(\tilde{u}_{a}, \tilde{v}_{a}\right)(0)=(1,0)$, and for all $t$,

$$
\begin{aligned}
& \dot{\tilde{u}}_{a}(t)=a g_{0}^{\prime}\left(x_{a}(t-1)\right) \tilde{u}_{a}(t-1)=a g_{0}^{\prime}(\ldots) \tilde{v}_{a}(t) \\
& \dot{\tilde{v}}_{a}(t)=\dot{\tilde{u}}_{a}(t-1)=a g_{0}^{\prime}\left(x_{a}(t-2)\right) \tilde{u}_{a}(t-2)=
\end{aligned}
$$$$
=a g_{0}^{\prime}(\ldots) \tilde{u}_{a}\left(t-\frac{2}{3}\right)=-a g_{0}^{1}(\ldots) \tilde{u}_{a}(t) .
$$

Here we used the symmetry ((s)) from section I. 1 . For $a+\alpha$, we have that $\left(\tilde{u}_{a}, \tilde{v}_{a}\right)$ converges to the solution of the initial value problem $\dot{u}=\alpha v^{\prime}, \dot{v}=-\alpha u,(u, v)(0)=(1,0)$

uniformly on $[-1,0]$. Hence $\frac{1}{\dot{x}_{a}(0)} \dot{x}_{a}(t) \rightarrow \cos \left(\frac{3}{2} \pi t\right)$, uniformly for $t \in[-1,0]$. The definition of $P_{a, \phi_{a}^{*}}$ and proposition I.6.1 now imply the assertion.

COROLIARY 2. There exist $a \in\left(\alpha, a_{5}\right), \varepsilon>0, \delta>0$ and $r_{2}^{\prime}>0$ with the following properties.

(i) $0<\int_{-1}^{t} \tilde{\zeta}$ for all $\tilde{\zeta} \in \zeta+C_{\varepsilon},-1<t \leqq 0$.

(ii) $\left|g_{0}^{1}(\xi)-1\right|<\frac{\varepsilon}{3|\zeta|}$ for $-\delta<\xi<\delta$

(iii) $\quad\left|\lambda_{a}-\zeta\right|<\frac{\varepsilon}{3}$

(iv) $\left|\phi_{a}^{*}\right|+2|r|\left|\lambda_{a}\right|<\min \{\delta, \gamma\}$ for $-r_{2}^{\prime}<r<r_{2}^{\prime}$

(v) The solution $w:[-1, \infty) \rightarrow \underline{R}$ of

$\dot{w}(t)=a_{0}^{\prime}\left(x_{a}(t-1)\right) w(t-1), w_{0}=\lambda_{a}$,

satisfies $0<w_{2}$; or equivalently,

$0 .<\mathrm{D}_{2} \mathrm{X}_{\mathrm{a} 0}\left(2, \phi_{\mathrm{a}}^{*}\right) \lambda_{\mathrm{a}}$.

PROOF. 1. There exists $t_{\zeta} \in\left(-\frac{1}{3}, 0\right)$ with $0<\zeta$ on $\left[-1, t_{\zeta}\right), \zeta<0$ on $\left(t_{\tau}, 0\right)$. It follows that the map $[-1,0] \ni t \rightarrow \int_{-1}^{t} \zeta \in \underline{R}$

is positive and increasing on $\left(-1, t_{\zeta}\right]$, and decreasing on $\left(t_{\zeta}, 0\right]$. One computes $\int_{-1}^{0} \zeta>0$. Hence

$$
0<\int_{-1}^{t} \zeta \text { for all } t \in(-1,0] \text {. }
$$

2. Choose $\varepsilon>0$ such that for all $\tilde{\zeta} \in \zeta+C_{\varepsilon}, \tilde{\zeta}>0$ on $\left[-1,-\frac{1}{3}\right]$ and $\int_{-1}^{t} \tilde{\zeta}>0$ on $\left[-\frac{1}{3}, 0\right]$. For such $\tilde{\zeta}$, we obtain part (i) of the assertion.

Choose $\delta>0$ such that (ii) holds. Corollary 1 implies that there 
exist $a^{\prime} \in\left(\alpha, a_{5}\right)$ and $r_{2}^{\prime}>0$ with

$\left|\lambda_{a}-\zeta\right|<\frac{\varepsilon}{3}$ for all $a \in\left(\alpha, a^{\prime}\right)$

and

$\left|\phi_{a}^{*}\right|+2|r|\left|\lambda_{a}\right|<\min \{\delta, \gamma\}$ for all $a \in\left(\alpha, a^{\prime}\right), r \in\left(-r_{2}^{1}, r_{2}^{1}\right)$.

2. DEPARTURE OF SOLUTIONS FROM THE LOCAL UNSTABLE MANIFOID OF $P$

3. For $a_{5}>a+\alpha$, the solution $w_{a}:[-1, \infty)+\underline{R}$ of

$\dot{w}(t)=a g_{0}^{\prime} \cdot\left(x_{a}(t-1)\right) w(t-1), w_{0}=\lambda_{a} \cdot$.

converges to the solution $y:[-1, \infty) \rightarrow \underline{R}$ of

$\dot{y}(t)=\alpha_{Y}(t-1), \dot{y}_{0}=\zeta$,

uniformiy on $[-1,2]$. The result of part 1 yields $0<y$ on $(0,1]$.

Therefore $0<\dot{y}$ on $[1,2]$, and $0<y_{2}$.

For $a \in\left(\alpha, a_{5}\right)$ as in Corollary 2, we set

$g_{1}:=\operatorname{ag}_{0}$

and drop the index a from now on:

$\phi^{*}=\phi_{a}^{*}, x=x_{a}, r_{0}=r_{0}(a), r_{1}=r_{1}(a), N=N_{a}, \tau=\tau_{a}$,

$\underline{P}=\underline{P}_{a}, D=D_{a}, P=P_{a}, \lambda=\lambda_{a}$.

Instead of

$\xi_{a}^{2}$

we shall write

$$
\mu \text {. }
$$

we set

$$
\mathrm{x}_{1}:=\mathrm{x}_{\mathrm{a} 0}
$$

According to section XI. 1 there is a decomposition

$$
\text { (1) } \quad H=L^{u} \oplus L^{S}
$$

into $D P\left(\phi^{*}\right)$-invariant closed subspaces. We have

$$
L^{\mathrm{U}}=\underline{R} \lambda
$$

and

$$
\mathrm{DP}\left(\phi^{*}\right) \lambda=\mu \lambda
$$

where $\mu>1$.

Let $p^{u}$ and $p^{S}$ denote the projections onto $L^{u}$ and $L^{S}$, given by (1). Occasionally we shall write $\phi^{\mathrm{L}}$ for $p^{\mathrm{u}} \phi, \phi^{\mathrm{s}}$ for $p^{\mathrm{s}} \phi$.

There is an equivalent norm ||$^{*}$ on $\mathrm{H}$ with respect to which $D F\left(\phi^{*}\right)$ induces an expansion on $I^{u}$ and a contraction on $I^{s}$.

For $M \subset E$ and $r>0$, we set

$$
M_{r}^{*}:=\left\{\phi \in M:|\phi|^{*}<r\right\} \text {. }
$$

An application of Theorem II.1.1 to $P$ yields radii $r(u), p(u)$, $r(s), p(s)$ and $c^{1}$-maps $u, s$; a one-dimensional local unstable manifold $U$ of $\phi^{*}$, a local stable manifold $S$ of $\phi^{*}$ with codimension 1 , and an open neighborhood $\mathrm{N}^{\mathrm{u}}$ corresponding to $\mathrm{D}^{\mathrm{u}}$ in Theorem II.1.1.

COROLIARY 1. For every solution $y:[-1, \infty) \rightarrow \underline{R}$ of eq. $\left(g_{1}\right)$ with $y_{0} \in S$,

$$
|y|<\frac{\gamma}{2} \text { on }[-1, \infty) \text {. }
$$

PROOF. Corollary II.1,1 says that $y_{0}$ defines a forward trajectory of $P$. The definition of $P, \tau(N) \subset(1,2), r_{1}<r_{0}$ and the choice of $r_{0}$ (Section I.7) imply the assertion.

COROLLARY 2. Let $\rho>0$ with $[-\rho, \rho] \lambda \subset I_{r(u)}^{u *}$ be given. There exists $\rho^{\prime} \in(0,0)$ such that for every open subset

$$
\begin{aligned}
\Delta \supset \phi^{*}+\{\phi+u(\phi): & \left.\phi \in\left[\rho^{\prime}, \rho\right] \lambda\right\} \text { of } H \\
(\cdots & \left.\left.\in\left[-\rho,-\rho^{\prime}\right] \lambda\right\} \text { of } H\right)
\end{aligned}
$$

there is $r(\Delta) \in\left(0, r_{1}\right)$ with the following properties.

(i) $\quad p^{s} H_{r(\Delta)}^{*} \subset I_{r(s)}^{S *}$ 
(ii) For every solution $y:[-1, \infty) \rightarrow \underline{R}$ of eq. $\left(g_{1}\right)$ with

$$
\begin{aligned}
\mathrm{y}_{0} \in \phi^{*}+\mathrm{H}_{\mathrm{r}}^{*}(\Delta) & \text { and }\left[\mathrm{y}_{0}-\phi^{*}\right]^{\mathrm{u}}-\mathrm{s}\left(\left[\mathrm{y}_{0}-\phi^{*}\right]^{\mathrm{s}}\right) \in(0, \infty) \lambda \\
(\ldots & \text { and } \ldots
\end{aligned}
$$

there exists $t(\Delta)=t\left(\Delta, y_{0}\right)>0$ with

$$
|y|<\frac{\gamma}{2} \text { on }[-1, t(\Delta)] \text { and } y_{t(\Delta)} \in \Delta \text {. }
$$

PROOF. Iet $\rho>0$ be given as above. 'Consider $\rho$ ' according to Proposition II.1.1. Let $\Delta$ be given as above. Consider $r(\Delta)$ according to Proposition II.1.1. We may assume $r(\Delta)<r_{1}$. Property (i) is satisfied.

Let $\mathrm{y}:[-1, \infty) \rightarrow \underline{\mathrm{R}}$ be a solution of eq. $\left(g_{1}\right)$ with

$$
\begin{aligned}
& \phi:=y_{0} \in \phi^{*}+H_{r}^{*}(\Delta) \text { and }\left[\phi-\phi^{*}\right]^{u}-s\left(\left[\phi-\phi^{*}\right]^{S}\right) \in(0, \infty) \lambda \\
& (\ldots \text { and } \ldots \text { a } \in(-\infty, 0) \lambda)
\end{aligned}
$$$$
\text { Then there are points } \phi_{0}=\phi, \phi_{1}, \ldots, \phi_{j}, j \in \underline{\mathbb{N}} \text {, in } D \text { with }
$$$$
\phi_{k+1}=P\left(\phi_{k}\right) \text { for } k=0, \ldots, j-1
$$

and

$$
\phi_{j} \in \Delta \text {. }
$$

set $t(\Delta):=\sum_{k}^{j-1} \tau\left(\phi_{k}\right)$. The definition of $P, \tau(\mathbb{N}) \subset(1,2)$

$r_{1}<r_{0}$ and properties of $r_{0}$ (Section 1.7 ) imply the remaining part of the assertion.

Corollary 2 will be used in section 3 below.

The next result describes solutions $y:[-1, \infty) \rightarrow \underline{R}$ of eq. $\left(g_{1}\right)$ which start on $U$ close to $\phi^{*}$. Then $y(0)=0=x(0)$. It is natural to expect $y_{t}>x_{t}$ (or $y_{t}<x_{t}$ ) after a while, for the following reason.

The difference $y_{0}-\phi^{*}$ is close to a multiple of the tangent vector to $U$ at $\phi^{*} ; \lambda$ is close to $\zeta$ (Corollary I.1); $\zeta$ is positive on "most" of $[-1,0]$; pairs of initial conditions with positive difference define solutions with increasing difference (Section $I .7$ ).

PROPOSIIION 1. There exists $r_{2}>0$ with

(i) $\quad\left(-r_{2}, r_{2}\right) \lambda \subset L_{i s}^{u *}(u)$

such that

(ii) for every solution $y:[-1, \infty) \rightarrow \underline{R}$ of eq. $\left(g_{1}\right)$ with $\mathrm{y}_{0}=\phi^{*}+\phi+u(\phi)$ and $\phi \in\left(0, r_{2}\right) \lambda$ (and $\left.\phi \in\left(-r_{2}, 0\right) \lambda\right)$, $(i i, 2) \quad y>x$ on $(0,2] \quad(y<<x$ on $(0,2])$.

PROOF. 1. $u(0)=0$ and $D u(0)=0$ permit to find $r_{2} \in\left(0, r_{2}^{1}\right) \cap(0,1)$ with (i) and

$$
|u(r \lambda)| \leqq|r \lambda| \text { min }\left\{1, \frac{\varepsilon}{3+\lambda \mid}\right\} \text { for }-r_{2}<r_{1} \leq r_{2}
$$

2. Let $r \in\left(-r_{2}, r_{2}\right), \phi:=r \lambda$. Consider the solution $y:[-1, \infty) \rightarrow \underline{R}$ with $y_{0}=\phi^{*}+\phi+u \overline{(\phi) \in} u \subset \phi^{*}+C_{r_{0}}$. The choice of $r_{0}$ (Section I.7) implies $|y|<\frac{\gamma}{2}$ on $[-1,2]$. This proves assertion (ii.1).

3. Eq. $\left(g_{1}\right), g_{1}=a g_{0}$ (see section 1 ) and $y(0)=0=x(0)$ yield

$$
y(t)-x(t)=a \int_{-1}^{t-1}\left\{g_{0} \circ\left[\phi^{*}+r \lambda+u(r \lambda)\right]-g_{0} \circ \phi^{*}\right\}
$$

for $0 \leqq t \leqq 1$.

The Mean Value Theorem implies that for every $v \in[-1,0]$ there exists $\theta_{\mathrm{V}} \in[0,1]$ with

$$
\begin{aligned}
& g_{0}([\ldots](v))-g_{0}\left(\phi^{*}(v)\right)-r \zeta(v)= \\
& g_{0}^{1}\left(\phi^{*}(v)+\theta_{v}[r \lambda+u(r \lambda)](v)\right)[\ldots](v)-r \zeta(v)= \\
& g_{0}^{1}(\ldots)[r \lambda(v)-r \zeta(v)]+\left(g_{0}^{1}(\ldots)-1\right) r \zeta(v)+g_{0}^{\prime}(\ldots) u(r \lambda)(v) .
\end{aligned}
$$

The argument of $g_{0}^{\prime}$ is bounded by $\min \{\delta, \gamma\}$ (see part 1 and corollary 1.2 (iv)) so that corollary 1.2 (ii) yields

$$
\left|g_{0}^{\prime}(\ldots)\right| \leqq 1 \text { and }\left|g_{0}^{\prime}(\ldots)-1\right|<\frac{\varepsilon}{3|\zeta|} \text {. }
$$

with Corollary 1.2 (iii), we obtain

$$
\left|g_{0}([\ldots](v))-g_{0}\left(\phi^{*}(v)\right)-r \zeta(v)\right|<|r| \frac{\varepsilon}{3}+\frac{\varepsilon}{3|\zeta|}|r||\zeta|+|r \lambda| \frac{\varepsilon}{3|\lambda|}
$$$$
=|r| \varepsilon \text {. }
$$

In case $0<r<r_{2}$, division by $r$ yields

$$
\left|\frac{1}{r}\left(g_{1}([\ldots](v))-g_{1}\left(\phi^{*}(v)\right)\right)-\zeta(v)\right|<\varepsilon \text { on }[-1,0] \text {. }
$$

With Corollary 1.2 (i), we infer

$$
0<\frac{1}{r} \int_{-1}^{t-1}\{\ldots\} \text { on }(0,1]
$$

which implies

$$
y>x \text { on }(0,1 I \text {. }
$$

For some $t \in(1,2), y_{t}>x_{t}$. The choice of $a_{5}$ in section. I.7, $g_{1}=$ $\mathrm{ag}_{0}$ with $\alpha<a<a_{5}$ and assertion (ii.1) permit to apply Proposition I.7.1 (i); we obtain $y_{2}>x_{2}$. 
In case $-x_{2}<r<0$, we find

$$
0<\frac{1}{r} \int_{-1}^{t-1}\{\ldots\} \text { for } 0<t \leqq 1 \text {; }
$$

hence

$$
a \int_{-1}^{t-1}\{\ldots\}<0
$$

and

$$
y<x \text { on }(0,1]
$$

As above, one concludes that $y<x$ on all of $(0,2]$
3. INTERSECTING H ABOVE AND BELOW THE LOCAL STABIE MANIFOLD

Set $0:=\left\{x_{t}: t \in \underline{R}\right\}$. Recall $|x|<\frac{\gamma}{4}$.

REMARK 1 . Our aim is to change $g_{1}$ outside the interval $[-\gamma, \gamma]$ to a periodic function $g$ which is sine-like in the sense that there is only one zero in the period interval $(0, w)$, in such a way that there is a heteroclinic solution $h: \underline{R} \rightarrow \underline{R}$ of eq. $(g)$ :

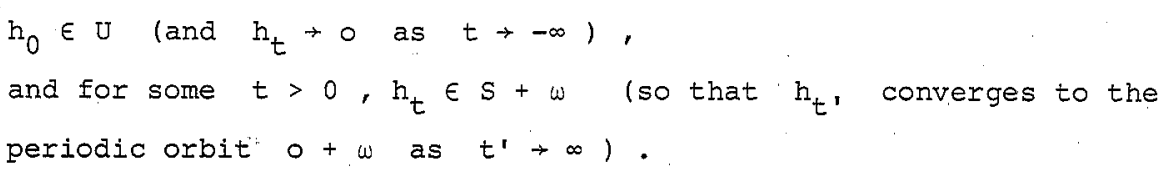

$h_{0}$ will then become a homoclinic point of a modification $P^{\prime}$ of $P$ which on a suitably small neighborhood of $h_{0}$ in $H$ is given by following the semiflow $x$ of eq. (g) until it reaches $H+w$, and by $a$ translation modulo $\omega$ back to $\mathrm{H}$.

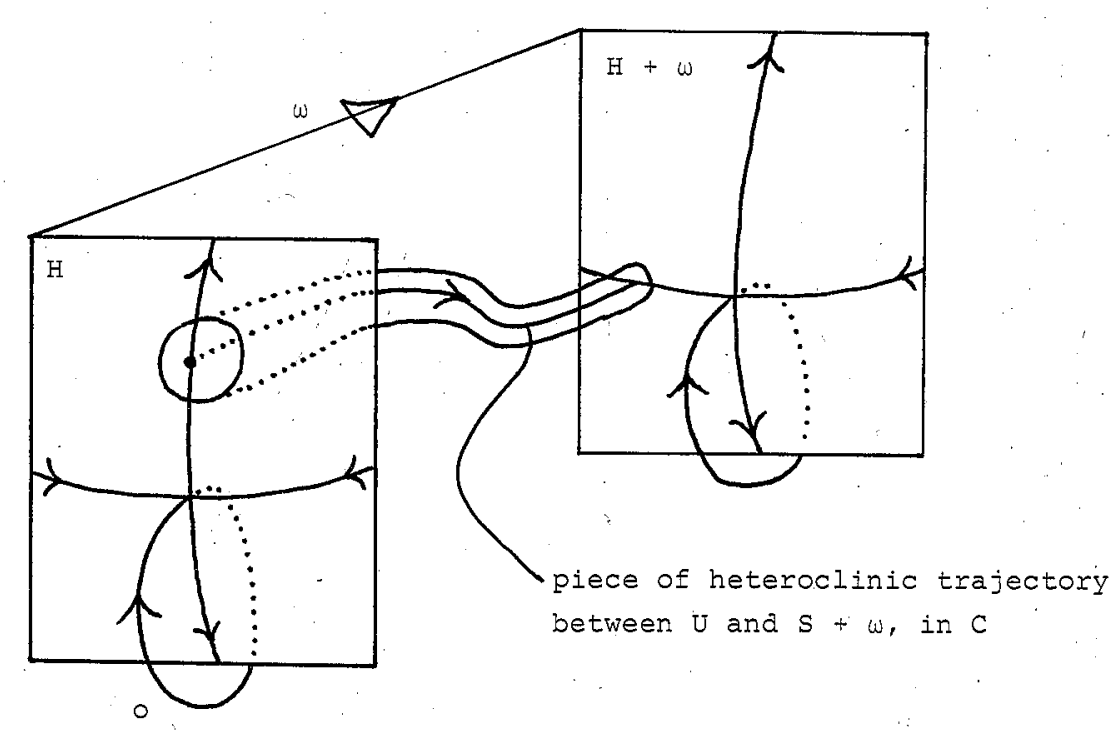




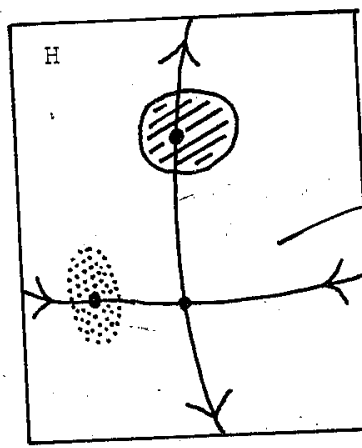

with the following properties.

For every solution $\mathrm{y}:[-1, \infty) \rightarrow \underline{\mathrm{R}}$ of eq. $\left(g_{1}\right)$ with $\mathrm{y}_{0}=\phi \in \mathrm{N}_{+}$,

$$
|y|<\frac{\gamma}{2} \text { on }[-1,2] \text { and }\left[y_{\tau(\phi)}-\phi^{*}\right]^{S} \in L_{r(s)}^{S *} \text {. }
$$

and $\mathrm{y}_{\tau(\phi)}$ is above, on or below $S$.

In case . $\phi^{*} \leqslant \phi\left(-\phi_{0} \leqslant \phi^{*}\right), \mathrm{Y}_{\tau(\phi)}$ is above (below) $S$, and there exists $\tilde{t}=\tilde{t}(\phi)>0$ with

$$
|y|<\gamma \text { on }[-1, \tilde{t}) \text { and } y(\tilde{t})=\gamma \quad(\ldots \text { and } y(\tilde{t})=-\gamma \cdot) \text {. }
$$

In case $\mathrm{y}_{\tau(\phi)} \in \mathrm{S}$,

$$
|y|<\frac{\gamma}{2} \text { on }[-1, \infty) \text {. }
$$

Let us defer the discussion of transversality of the homoclinic point 4 , and concentrate on the search for $g$, with a heteroclitwill be a submanifold of codimension nic solution. The target set $S+w$ will be a a $\phi^{*}+\omega$ in1 in the hyperplane $H+w$ which divides a neighborhood of not likely that to upper and lower halves, both open in. $\mathbb{E}+w$. It one can construct $g$ so that a solution $h$ reaches the thin" set $s+\omega$; it will certainly be easier to find $g$ so that a solution reache, the open halves above and below $s+w$. This is what we with an application of the Intermediate value

Let $\phi \in F$ with $\left[\phi-\phi^{*}\right]^{S} \in I_{r(s)}^{S *}$ be given. $\phi$ is said to DEFINITION. Let $b \in$ (below) $S$ if and only if

lie above (below) $s$ if and only $s^{*},(0, \infty) \lambda$

$$
\begin{gathered}
{\left[\phi-\phi^{*}\right]^{\mathrm{u}}-s\left(\left[\phi-\phi^{*}\right]^{\mathrm{s}}\right) \in(0, \infty) \lambda} \\
(\ldots \quad \ldots \in(-\infty, 0) \lambda)
\end{gathered}
$$

Clearly $\phi \in S$ is equivalent to

$$
\left[\phi-\phi^{*}\right]^{u-s}-s\left(\left[-\phi^{*}\right]^{s}\right)=0 \text {. }
$$

The next result provides inequalities for initial values close to $\phi^{*}$ that above or below $s$. This will fawhich guarantee that solutions reach grof uses corollary Croposition II.1.1 on attraction $2.2,1 . e .$, a consequence of the technical proposita to subsets of local unstable manlfolds, togectic for. $\left(g_{1}\right)$. parts of section I.7 whlch are specific results for $\mathrm{gq} \cdot\left(g_{1}\right)$ 
with $\rho^{\prime}=\rho^{+}$. We show that there exists an open neighborhood $\Delta^{+}$of $\left.\phi^{*}+\phi^{+} u(\phi): \phi \in\left[\rho^{+}, \rho\right] \lambda\right\}$ in $C$ such that for every solution $y:[-1, \infty) \rightarrow \underline{R}$ of eq. $\left(g_{1}\right)$ with $y_{0} \in \Delta^{+}$,

$|y|<\frac{y}{2}$ on $[-1,2]$ and $x<y$ on $[1,2]$ : Because of $\rho<r_{2}$, Proposition 2.1 implies that for every $\tilde{\phi}$ in $\phi^{*}+\left\{\phi+u(\phi): \phi \in\left[\rho^{+}, \rho\right] \lambda\right\}$ there is an open neighborhood $\tilde{\Delta}$ in $C$ with

$|y|<\frac{y}{2}$ and $x<y$ on $[1,2]$ for every solution $y:[-1, \infty) \rightarrow \underline{R}$

of eq. $\left(g_{1}\right)$ with $y_{0} \in \tilde{\Delta}$.

2. Similarly, there is $p^{-} \in(0,0)$ so that the assertion in brackets of Corollary 2.2 holds true, with $\rho^{\prime}=\rho^{-}$, and there is an open neighborhhod $\Delta^{-}$of $\phi^{*}+\left\{\phi+u(\phi): \phi \in\left[-\rho,-\rho^{-}\right] \lambda\right\}$ with

$|y|<\frac{\gamma}{2}$ on $[-1,2]$ and $x>y$ on $[1,2]$ for all solutions $y:[-1, \infty) \rightarrow \underline{R}$ of eq. $\left(g_{1}\right)$ with $y_{0} \in \Delta^{-}$.

3. Corollary 2.2 , applied to $\Delta^{+} \cap \mathrm{H}$ and to $\Delta^{-} \cap \mathrm{E}$, yields existence of $r^{+}=r\left(\Delta^{+} \cap H\right)$ and $r^{-}=r\left(\Delta^{-} \cap H\right)$ in $\left(0, r_{1}\right)$ with

(*) $\left[\phi-\phi^{*}\right]^{S} \in I_{r(s)}^{S *}$ for all $\phi \in \phi^{*}+\mathrm{H}_{r^{*}}^{*}$

where $r^{*}:=\min \left\{r^{+}, r^{-}\right\}$

such that for every solution $y:[-1, \infty) \rightarrow \underline{R}$ of eq. $\left(g_{1}\right)$ with

$$
\begin{array}{r}
\mathrm{y}_{0} \in \phi^{*}+H_{r^{*}}^{*} \text { and }\left[\mathrm{y}_{0}-\phi^{*}\right]^{\mathrm{u}}-s\left(\left[\mathrm{y}_{0}-\phi^{*}\right]^{s}\right) \in(0, \infty) \lambda \\
\in(-\infty, 0) \lambda
\end{array}
$$$$
(\ldots \quad \text { and } \ldots \quad \in(-\infty, 0) \lambda)
$$

there exists $t(y)=t\left(\Delta^{+} \cap H, y_{0}\right)>0\left(\ldots t(y)=t\left(\Delta^{-} \cap H, \mathrm{y}_{0}\right)>0\right)$ with

$$
|y|<\frac{\gamma}{2} \text { on }[-1, t(y)) \text { and } y_{t(y)} \in \Delta^{+} \cap H \quad\left(\ldots \in \Delta^{-} \cap H\right) \text {. }
$$

4. Corollary 2.1 yields $|y|<\frac{\gamma}{2}$ on $[-1, \infty)$ for solutions $\mathrm{Y}:[-1, \infty)+\underline{R}$ of eq. $\left(g_{1}\right)$ with $\mathrm{y}_{0} \in \phi^{*}+\mathrm{H}_{\mathrm{r}^{*}}^{*}$ and $\mathrm{y}_{0} \in \mathrm{s}$; i.e.,

$$
\left.\left[y_{0}-\phi^{*}\right]^{u-s}-s\left(y_{0}-\phi^{*}\right]^{s}\right)=0 \text {. }
$$

5. Parts $3,1,2$ above imply that for solutions $y:[-1, \infty) \rightarrow \underline{R}$ of eq. $\left(g_{1}\right)$ with $y_{0} \in \phi^{*}+H_{r^{*}}^{*}$ and $\left[y_{0}-\phi^{*}\right]^{u}-' s\left(\left[y_{0}-\phi^{*}\right]^{s}\right) \in(0, \infty) \lambda$ $(\ldots \in(-\infty, 0) \lambda)$, $(-\infty, 0) \lambda)$,
$|y|<\frac{\gamma}{2}$ in $[-1, t(y)+2]$ and $x<y$ in $[t(y)+1, t(y)+2]$
$(\ldots$
Recall $|x|<\frac{\gamma}{4}$. Proposition I.7.1 (ii) yields, existence of $t^{*}=t^{*}(y)$ in $(t(y)+2, \infty)$ with $|y|<\gamma$ in $\left[-1, t^{*}\right)$ and $y\left(t^{*}\right)=\gamma(\ldots=-\gamma)$. 6. Choose an open neighborhood $\mathbb{N}_{+} \subset \mathrm{N}$ of $\phi^{*}$ in $C$ so small that $N_{+} \subset C_{\frac{\gamma}{2}}$ (recall $\left.|x|<\frac{\gamma}{4}\right)$, and for every solution $y:[-1, \infty) \rightarrow \underline{R}$ of eq. $\left(g_{1}\right)$ with $y_{0} \in N_{+}$,

$$
|y|<\frac{\gamma}{2} \text { on }[-1,2] \text { and } y_{\tau\left(y_{0}\right)} \in \phi^{*}+H_{Y^{*}}^{*}
$$

(recall $\left.1<\tau\left(\mathrm{y}_{0}\right)<2\right)$.

For such solutions, $\left[y_{\tau\left(y_{0}\right)}-\phi^{*}\right]^{s} \in L_{r(s)}^{S *}$, see $(*), ;$ and $y_{\tau\left(y_{0}\right)}$ is above, on or below $S$.

The case $x_{0}<y_{0} \in \mathbb{N}_{+}$: Proposition.I.7.1 (ii) implies that for some $\tilde{t}=\tilde{t}\left(y_{0}\right)>0,|y|<\gamma$ in $[-1, \tilde{t})$ and $y(\tilde{t})=\gamma$. By the choice of $N_{+}$ $\tilde{t}>2>\tau\left(y_{0}\right) \cdot y_{\tau\left(y_{0}\right)} \in \phi^{*}+H_{r^{*}}^{*}$ is above, on or below $S$.

Assume "below". Then the solution $\tilde{y}:[-1, \infty) \rightarrow \underline{R}$ of eq. $\left(g_{1}\right)$ with

$\tilde{y}_{0}=y_{\tau\left(y_{0}\right)}$ satisfies $|\tilde{y}|<\gamma$ in $\left[-1, t^{*}(\tilde{y})\right)$ and $\tilde{y}(t *(\tilde{y}))=-\gamma$, see part 5. With $\tilde{Y}=y\left(\cdot+\tau\left(y_{0}\right)\right) \mid[-1, \infty)$, we obtain a contradiction to the properties of $\tilde{t}\left(y_{0}\right)$.

Assume "on". Then $|\tilde{Y}|<\frac{\gamma}{2}$ in $[-1, \infty)$, see part 4. This contradicts $\tau\left(y_{0}\right)<\tilde{t}\left(y_{0}\right)$ and $y\left(\tilde{t}\left(y_{0}\right)\right)=\gamma$.

It follows that $y_{\tau\left(y_{0}\right)}$ is above $S$.

The proof for the case $\mathbb{N}_{+} \ni \mathrm{y}_{0}<\mathrm{x}_{0}$ proceeds analogously.

The assertion for $y_{\tau\left(y_{0}\right)} \in S$ follows from $1<\tau\left(y_{0}\right)<2,|y|<\frac{\gamma}{2}$ in $[-1,2]$, and from part 4 .

REMARK 3. Having Propósition 1, we shall look for periodic extensions $g$ of $g_{1} \mid[-\gamma, \gamma]$ so that there are solutions $h$ of eq. (g) which start in $U$ and have segments $h_{t_{+}}>\phi^{*}+\omega\left(h_{t_{+}}<\phi^{*}+\omega\right), t_{+}>0$, in $N_{+}+\omega$ where $\omega$ is the minimal period of $g$. The next result prepares targets modulo $\omega$ for this, i.e., functions $\phi^{* *}+r>_{\phi^{*}}\left(\phi^{* *}-r<\phi^{*}\right)$, $r>0$, in $\mathrm{N}_{+}$for which it will be possible to achieve

$$
h_{t_{+}}(t)=\phi^{* *}(t)+r+\omega\left(\cdots=\phi^{* *}(t)-r+\omega\right),
$$

not immediately on all of $[-1,0]$ but first only on some subinterval 


$$
\text { I } \supset\left[-1,-\frac{1}{3}\right] \text {. }
$$

do not simply set $\phi^{* *}:=\phi^{*}$, for the following reason. A situaat $h\left(t_{+}-\frac{4}{3}\right)$ and at $h\left(t_{+}-2\right)$, because of eq. $\left(g_{1}\right)$ and $\dot{x}(-1)=0$ $\dot{x}\left(-\frac{1}{3}\right)=0$. This would at least present an additional difficulty in the search for sine-like functions $g$ with only one zero in the period interval $(0, \omega)$.

Instead, we modify $\phi^{*}$ in order to avoid the zero of $\dot{x}$ at -1

we choose $r_{3} \in\left(0, \frac{\gamma}{4}\right)$ such that

$$
\text { (1) } \quad \phi^{*}+\mathrm{C}_{2 \mathrm{x}_{3}} \subset \mathrm{N}_{+}
$$

and

(2) $\phi^{*}(-1)+2 r_{3}<0$.

PROPOSITION 2. There exists $t_{*} \in\left(-1,-\frac{2}{3}\right)$ such that for $\phi^{* *} \in C$ with $\psi^{*}(t)=\phi^{*}(t)$ in $\left[t_{*}, 0\right]$ and $\dot{\phi}^{* *}(t)=\dot{\phi}^{*}\left(t_{*}\right)$ in $\left[-1, t_{*}\right)$

$$
\text { (i) } \phi^{* *}-r_{3}<\phi^{*}<\phi^{* *}+r_{3} \text {. }
$$

In particular,

(ii) $\phi^{* *} \in \phi^{*}+C_{r_{3}}$ (iii) $\phi^{*}-2 r_{3}<\phi^{* *}-r_{3}<\phi^{*}<\phi^{* *}+r_{3}<\phi^{*}+2 r_{3}$.

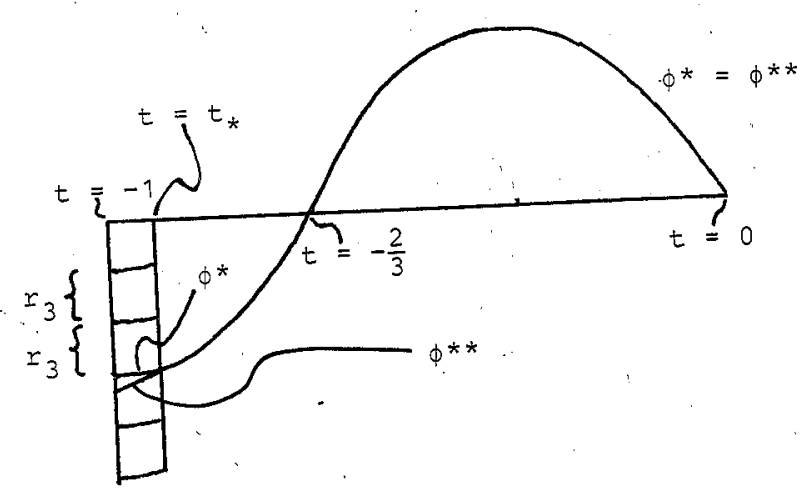

PROOF of Proposition 2. Recall $\dot{x}(-1)=0$. Choose $t_{*} \in\left(-1,-\frac{2}{3}\right)$ with

$0<\dot{x}\left(t_{*}\right)<r_{3}$ and $x\left(t_{*}\right)<x(-1)+r_{3}$.

Consider $\phi^{* *}$ as above. We have $\phi^{* *}(t)=\phi^{*}(t)$ in $\left[t_{*}, 0\right]$, and for $-1 \leq t<t_{*}$

$$
\phi^{*}(t)<\phi^{* *}\left(t_{*}\right)=x\left(t_{*}\right)<x(-1)+r_{3} \leqq x(t)+r_{3}
$$

(with $0<<$ in $\left(-1,-\frac{1}{3}\right)$ ), and

$$
\begin{array}{ll}
\phi^{* *}(t) \geqq \phi^{* *}(-1)=x\left(t_{*}\right)+\left(-1-t_{*}\right) \dot{x}\left(t_{*}\right) & \\
>x\left(t_{*}\right)+\left(-1-t_{*}\right) r_{3} & \left.\quad \text { (by the choice of } t_{*}\right) \\
>x(t)-r_{3} & \left(\text { with } 0<\dot{x} \text { in }\left(-1,-\frac{1}{3}\right)\right. \text { and } \\
& \left.-1-t_{*}>-1\right) .
\end{array}
$$

In order to deduce from $h_{t_{+}}(t)=\phi^{* *}(t)+r_{3}+\omega\left(\ldots=\phi * *(t)-r_{3}\right.$ $+\omega$ ) on some subinterval

$$
\text { I } \supset\left[-1,-\frac{1}{3}\right]
$$

of $[-1,0]$ that $h_{t_{+}} \in \phi^{*}+c_{2 r_{3}}+\omega$ and $h_{t_{+}}(t)>\phi^{*}(t)+\omega$

$\left(h_{t_{+}}(t)<\ldots\right)$ on all of $[-1,0]$, we need a further elementary consi-

\section{deration.}

We choose $r_{4} \in\left(0, r_{3}\right)$ so small that

(3) $\phi^{*}-2 x_{3}<\phi^{* *}-r_{3}-r_{4}<\phi^{* *}-r_{3}+r_{4}<\phi^{*}$ $<\phi^{* *}+r_{3}-r_{4}<\phi^{* *}+r_{3}+r_{4}<\phi^{*}+2 r_{3}$

and

(4) $\quad g_{1}\left(\frac{\gamma}{4}\right)+r_{4}<g_{1}(\gamma)$.

In addition, we fix -a constant $c_{1}<g_{1}(-\gamma)<0$. 


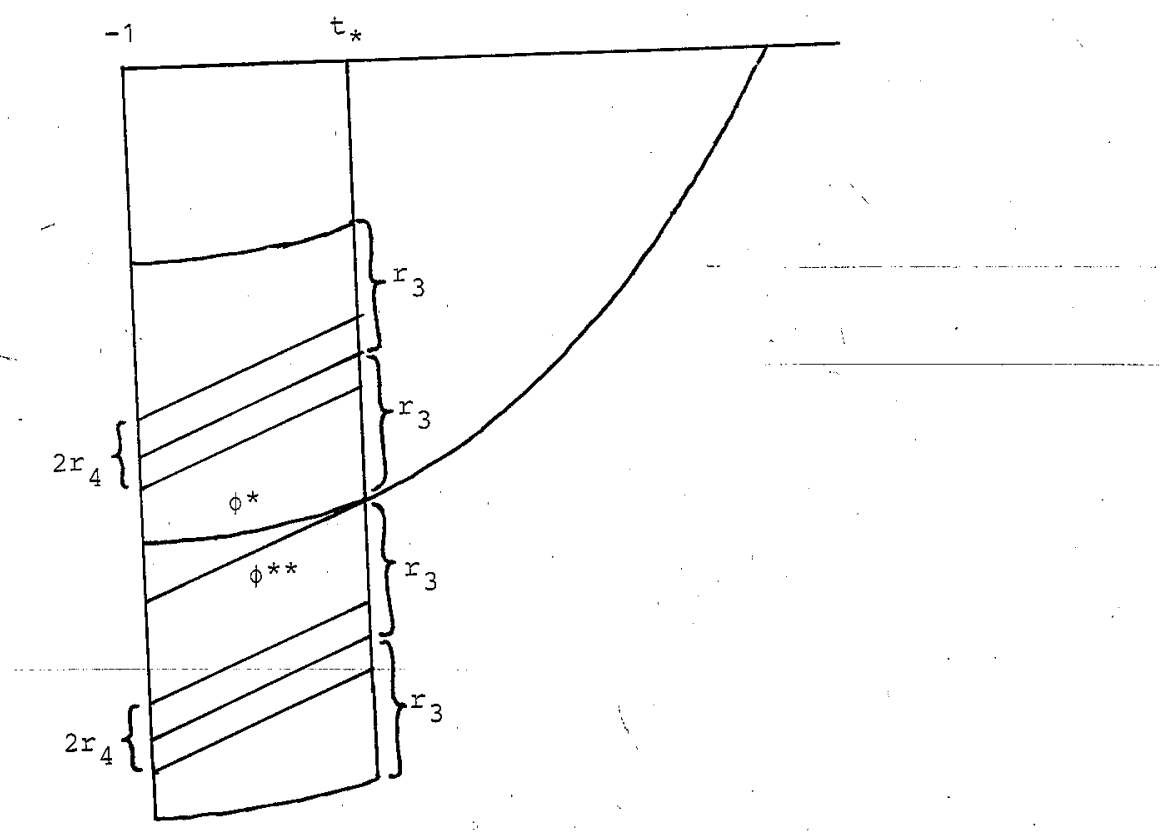

PROPOSITION 3. There exists $\theta \in\left(-\frac{1}{3}, 0\right)$ such that for every differentiable $\phi \in C$ with $c_{1}<\dot{\phi} \leqq 0$ on $\left[-\frac{1}{3}, 0\right]$ the following holds.

(i) If $x(\theta)-r_{3}-r_{4} \leqq \phi(\theta) \leqq x(\theta)+r_{3}+r_{4}$ then $x(t)-2 r_{3}<\phi(t)<x(t)+2 r_{3}$ for all $t \in[\theta, 0]$.

(ii) If $x(\theta)+r_{3}-r_{4} \leqq \phi(\theta)$ then $x(t)<\phi(t)$ for $\theta \leqq t \leqq 0$.

iii) If $\phi(\theta) \leqq x(\theta)-r_{3}+r_{4}$ then $\phi(t)<x(t)$ for $\theta \leqq t \leqq 0$.

pROOF. $r_{4}<r_{3}$ and $x(0)=0$ permit to choose $\theta \in\left(-\frac{1}{3}, 0\right)$ with

$$
0<\theta c_{1}<r_{3}-r_{4} \text { and } x(\theta)<r_{3}-r_{4} \text {. }
$$

Consider $\phi$ as above. For $\theta \leqq t \leqq 0$,

$\phi(\theta) \cdots \phi(t)=\phi(\theta)+\int_{\theta}^{t} \dot{\phi} \geqq \phi(\theta)+(t-\theta) c_{1}$.

If in adaition $\phi(\theta) \leqq x(\theta) \div r_{3}+r_{4} \quad\left\{\phi(\theta) \leqq x(\theta)-r_{3}+r_{4}\right\}$

then we obtain

$$
\begin{aligned}
& \phi(t) \leqq \phi(\theta) \leqq x(\theta)+r_{3}+r_{4}<2 r_{3} \quad \text { (by the choice of } \theta \text { ) } \\
& \leqq x(t)+2 r_{3}
\end{aligned} \quad \text { (by } 0 \leqq x \text { in }\left[-\frac{2}{3}, 0\right] \text { ) }
$$

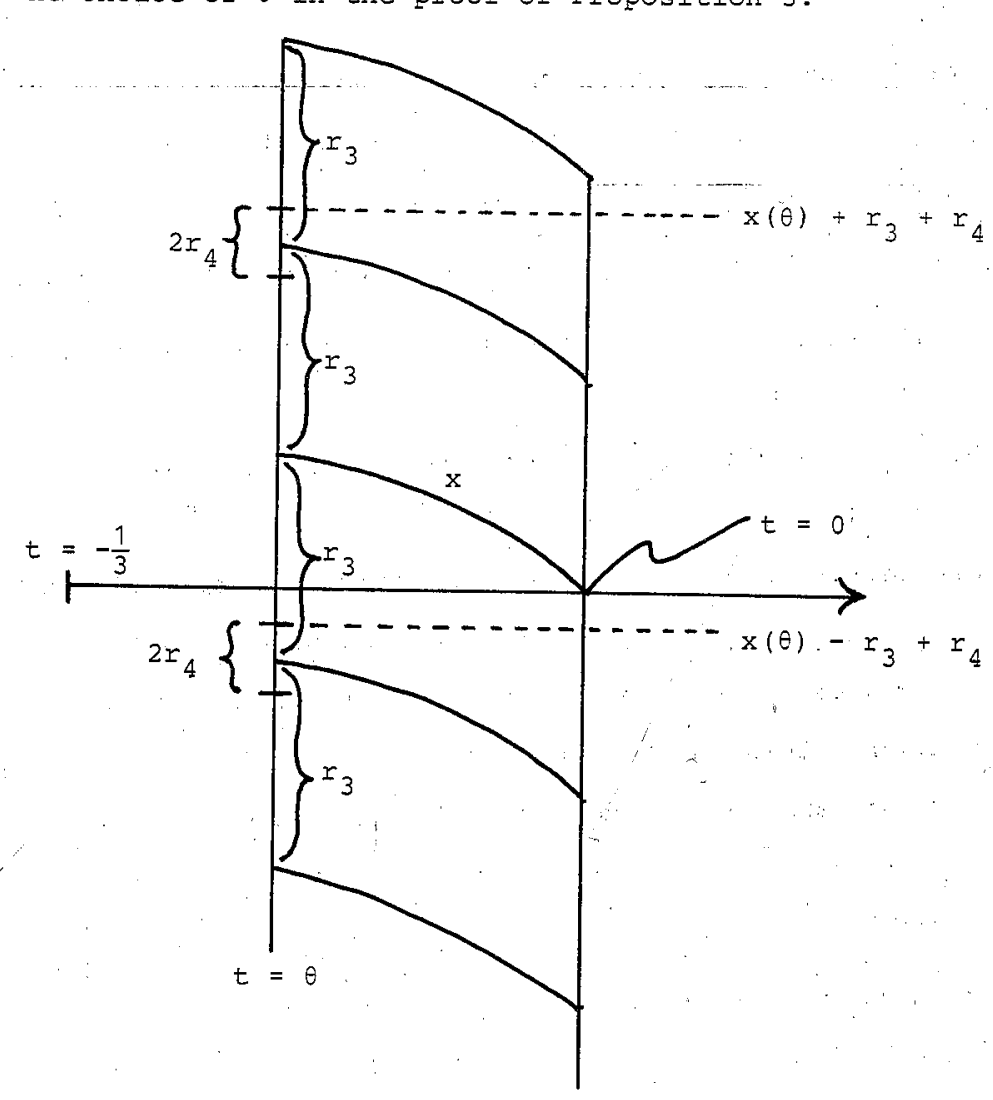

Proof of the lower estimate: If in addition

$$
x(\theta)-r_{3}-r_{4} \leqq \phi(\theta) \quad\left\{x(\theta)+r_{3}-r_{4} \leqq \phi(\theta)\right\}
$$

then

$$
\phi(t) \geqq x(\theta)-r_{3}-r_{4}-\theta c_{1}>x(t)-2 r_{3}
$$

(with the choice of $\theta$, and with $\dot{x}<0$ on $\left(-\frac{1}{3}, 0\right]$ ) \{then

$$
\phi(t) \geqq x(\theta)+r_{3}-r_{4}-\theta c_{1}>x(t) .
$$

(with the choice of $\theta$, and $\dot{x}<0$ on $\left.\left(-\frac{1}{3}, 0\right]\right)$. 
4. A CONDITION FOR TRANSVERSALITY

Recall that we want to find a periodic extension $g$ of $-g_{1} \mid[-\gamma-\gamma\} \cdots$, with period $w$, such that there is a heteroclinic solution $h$, with $x:=h_{0} \in U$ and $h_{t_{+}} \in \mathbb{N}_{+}+w$ for some $t_{+}>0$, so that after an additional time of flight $\tau\left(h_{t_{+}}-\omega\right)$ the segment $h_{t} \in C$ reaches $s+\omega$ in $H+\omega$. In terms of the modified poincare'map $P^{\prime}$ from Remark 3.1 ,

$P^{\prime}(x) \in S$.

The transversality property we have in mind includes

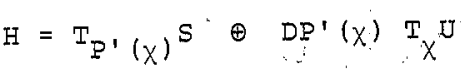

or equivalently, since $\mathrm{T}_{\mathrm{P}^{\prime}}(x)$ S has codimension 1 while dim $\mathrm{T}_{\chi} \mathrm{U}=1$,

$D P^{\prime}(X) v \notin T_{P^{\prime}(X)} S$ for some nonzero $v \in T_{X} U^{U}$.

In the present section we look for sufficient (in fact, equivalent) conditions for this which do not involve $P, P^{\prime}$ or $\tau$ and which can be expressed in terms of the nonlinearity $g$ alone.

For $t_{+}$as above, there is a decomposition

$$
D P^{\prime}(x)=D P^{\prime}\left(h_{t_{+}}-\omega\right) \circ D_{2} X\left(t_{+} ; x\right) \text {, }
$$

because of periodicity; recali $\underline{P}=\underline{P}_{a}$ and $\underline{P}_{a}(\phi)=X_{a 0}\left(\tau_{a}(\phi), \phi\right)$ on $N_{a}=N \supset N_{+}$. Let $v \in T_{X} U$ - $\{0\}$, be given. Since $h_{t_{+}}-\omega \in N_{+}$in the last formula and $D_{2} X\left(t_{+}, x\right) v \in C$ are obtained from $h$ and from the linear variational equation along $h$, i.e., both in terms of $g$ and $g^{r}$, we only look for a suitable condition on points $\dot{y}_{0} \in \mathbb{N}_{+}$with $\underline{p}_{0}\left(\mathrm{y}_{0}\right) \in S$ and on vectors ' $w_{0} \in C$ which ensures

$$
\left.\underline{D} \underline{\underline{P}}\left(\mathrm{y}_{0}\right) \mathrm{w}_{0} \quad \notin \quad \mathrm{T}_{\underline{\underline{P}}\left(\mathrm{y}_{0}\right)}\right)^{\mathrm{S}} \text {. }
$$

PROPOSITION 1. Let $\mathrm{Y}:[-1, \infty) \rightarrow \underline{\mathrm{R}}$ be a solution of eq. $\left(g_{1}\right)$ with $\mathrm{y}_{0} \in \mathrm{N}_{+}$ and $\underline{P}\left(y_{0}\right) \in S$. Let $w:[-1, \infty) \neq \underline{R}$ be a solution of

$$
\dot{w}(t)=g_{1}^{\prime}(y(t-1) w(t-1) \text {. }
$$

If there exists $t \geqq 0$ with $w\left(t^{\prime}\right) \neq 0$ for all $t^{\prime} \in[t-1, t]$ ' then

$$
\mathrm{D} \underline{\underline{P}}\left(\mathrm{Y}_{0}\right) w_{0} \notin \mathrm{T}_{\underline{\underline{P}}}\left(\mathrm{y}_{0}\right)^{S} \text {. }
$$

Moreover, the forward iterates of $x_{1}:=\underline{P}\left(y_{0}\right)$ and $v_{1}:=D \underline{P}\left(y_{0}\right) w_{0}$ given by $x_{n+1}=P\left(x_{n}\right) \in S$ and $v_{n+1}=D P\left(x_{n}\right) v_{n}$ for $n \in \underline{N}$ satisfy

$$
v_{n} \notin T_{X_{n}} s \text { for all } n \in \underline{N} \text {. }
$$

PROOF. 1. Assume $0<w$ in $[t-1, t]$ for some $t \geq 0$. (In case $w_{t}<0$, consider - $w$ instead of $w$.$) By Proposition 3.1, |\mathrm{y}|<\frac{\gamma}{2}$ on $[-1, \infty)$, and the coefficient in the linear variational equation along $y$ is bounded from below by $g_{1}^{\prime}\left(\frac{\gamma}{2}\right)>0$. With $0<w_{t}$, we infer $w\left(t^{\prime}\right) \rightarrow \infty$ as $t^{\prime} \rightarrow \infty$

2. Define $t_{1}:=\tau\left(y_{0}\right)$ and $t_{n+1}:=t_{1}+\sum_{k}^{n} \tau\left(x_{k}\right)$ for $n \in \mathbb{N}$. Then $x_{n}=x_{1}\left(t_{n}, y_{0}\right)=y_{t_{n}}$ for all $n \in \underline{\mathbb{N}}$,

$v_{n}=D\left(P^{n-1} \circ \underline{P}\right)\left(y_{0}\right) w_{0}=p(n) \circ D_{2} x_{1}\left(t_{n}, y_{0}\right) w_{0}$ for all $n \in \underline{N}$,

where $p_{(n)} \phi=\phi-\frac{\phi(0)}{\dot{y}\left(t_{n}\right)} \dot{y}_{t_{n}}$ for all $\phi \in C$ (compare section 1).

3. Let $\mathrm{n} \in \underline{\mathbb{N}}$. Consider the solutions $\mathrm{y}:[-1, \infty) \rightarrow \underline{\mathrm{R}}$ of eq. $\left(g_{1}\right)$ with $\mathrm{y}_{0}=x_{n}=y_{t_{n}}$, and $\underline{w}:[-1, \infty)+\underline{R}$ of

$$
\dot{w}\left(t^{\prime}\right)=g_{1}^{\prime}\left(y\left(t^{\prime}-1\right)\right) w\left(t^{\prime}-1\right), w_{0}=v_{n} \text {. }
$$

We show that there exists $\underline{t}>0$ with $\underline{w}>0$ on $[\underline{t}-1, \underline{t}]$ :

From $y=y\left(\cdot+t_{n}\right) \mid[-1, \infty)$ we see that $\underline{w}, w\left(\cdot+t_{n}\right)$ and $\dot{y}\left(\cdot+t_{n}\right)$ satisfy the same linear variational equation on $\underline{R}^{+}$. Part 2 implies

$$
\underline{w}_{0}=v_{n}=p(n) w_{t_{n}}=w_{t_{n}}-\frac{w\left(t_{n}\right)}{\dot{y}\left(t_{n}\right)} \dot{y}_{t_{n}}
$$

for the initial values. Hence

$$
\underline{w}=w\left(\cdot+t_{n}\right)-\frac{w\left(t_{n}\right)}{\dot{y}\left(t_{n}\right)} \dot{y}\left(\cdot+t_{n}\right) \text { on }[-1, \infty) .
$$

Note $\dot{y}\left(t^{\prime}+t_{n}\right) \in g_{1}\left(\left[-\frac{\gamma}{2}, \frac{\gamma}{2}\right]\right)$ for $-1 \leqq t^{\prime}$. Recall part 1 .

4. Suppose $v_{n} \in T_{X_{n}} s^{\prime}$. There is a $C^{1}$-curve $\rho:(-1,1) \rightarrow H$ with $\rho((-1,1)) \in s, \rho(0)=x_{n}, D \rho(0) 1=v_{n} \cdot 0<\underline{w}_{t}$ gives $0<D_{2} x_{1}(t, \rho(0)) \underline{w}_{0}$. Continuity implies that there are a constant function $\phi>0$ in $C$ and $\varepsilon>0$ such that

$$
\bar{D}_{2} X_{1}(t, \rho(\cdot)) D \rho(\cdot), 1 \geqq \phi \text { on }[-\varepsilon, \varepsilon] \text {. }
$$

Let $\tilde{y}:[-1, \infty) \rightarrow \underline{R}$ denote the solution of eq. $\left(g_{1}\right)$ with $\tilde{y}_{0}=p(\varepsilon) \in S$. By Corollary $2.1,|\tilde{y}|<\frac{\gamma}{2}$ on $[-1, \infty) \ldots|y|<\frac{\gamma}{2}$ on $[-1, \infty)$ and $y(t)=y\left(t+t_{n}\right)$ for $t \geqq-1$ (see part 3 ) give $|y|<\frac{\gamma}{2}$ on $[-1, \infty)$, 
too. We obtain

$$
\tilde{y}_{\underline{t}}-\underline{y}_{\underline{t}}=x_{1}(\underline{t}, \rho(\varepsilon))-x_{1}(\underline{t}, \rho(0))
$$

$=\int_{0}^{\varepsilon} D\left(\varepsilon^{\prime \prime} \rightarrow \mathrm{X}_{1}\left(\underline{\underline{t}}, \rho\left(\varepsilon^{\prime \prime}\right)\right)\right)\left(\varepsilon^{\prime}\right) 1 \mathrm{~d} \varepsilon^{\prime}=\int_{0}^{\varepsilon} D_{2} \mathrm{X}_{1}\left(\underline{\underline{t}}, \rho\left(\varepsilon^{\prime}\right)\right) D \rho\left(\varepsilon^{\prime}\right) 1 \mathrm{~d} \varepsilon^{\prime}$

$\geqq \varepsilon \phi>0$

(with the monotonicity of the C-valued Riemann integral).

An application of Proposition I.7.1 (i) to the difference

$$
d:[-1, \infty) \ni t \rightarrow \tilde{Y}(t+\underline{t})-\underline{y}(t+\underline{t}) \in \underline{R}
$$

yields

$$
0<g_{j}^{\prime}(\gamma) \varepsilon \phi(0) \leqq \dot{d} \text { on }[-1, \infty),
$$

a contradiction to boundedness of $\tilde{Y}$ and $\underline{Y}$.

REMARK. The condition in Proposition 1 is also necessary for

$$
v_{n} \notin T_{X_{n}} S \text { for all } n \in \underline{N} \text {. }
$$

Sketch of proof. Let, $y, w$ be given as in Proposition 1. The points $x_{n}$ in $S$ tend to $\phi^{*}$ as $n \rightarrow \infty$. By an inclination lemma for maps which are not necessarily invertible (see $[8,36,18]$ ),

$$
\frac{1}{\mid v_{n}} v_{n} \rightarrow \pm \frac{1}{|\lambda|} \lambda \text { as } n \rightarrow \infty
$$

(Here one uses $v_{n} \notin T_{\chi_{n}}$ s.). Corollary $1.2(v)$ and continuity now imply that for $n$ sufficiently large, $D_{2} x_{1}\left(2, x_{n}\right) v_{n}$ has no zero in $[-1,0]$.

Consider $t_{n}:=\tau\left(y_{0}\right)+\sum_{k}^{n-1} \tau\left(x_{k}\right)$ as in the proof of proposition 1 . The solution $\tilde{w}:[-1, \infty)+\underline{R}$ of the linear variational equation along $y\left(\cdot+t_{n}\right)$ with $\tilde{w}_{0}=v_{n}$ satisfies $\left|\tilde{w}\left(t^{\prime}\right)\right| \rightarrow \infty$ as $t^{\prime} \rightarrow \infty$ because $\tilde{w}_{2}=D_{2} x_{1}\left(2, x_{n}\right) v_{n}$ has no zero and $g_{1}^{\prime}\left(y\left(\cdot+t_{n}-1\right)\right) \geqq g_{1}^{\prime}\left(\frac{\gamma}{2}\right)>0$ $w\left(\cdot+t_{n}\right)$ and $\dot{y}\left(\cdot+t_{n}\right)$ satisfy the same linear variational equation as wi and for the initial values we have

$$
\begin{aligned}
& \begin{aligned}
\begin{aligned}
v_{n} & =D\left(P^{n-1} \circ \underline{p}\right)\left(y_{0}\right) w_{0}=p(n) \circ D_{2} x_{1}\left(t_{n}, y_{0}\right) w_{0}
\end{aligned} \\
=w_{t_{n}}-\frac{w\left(t_{n}\right)}{\dot{y}\left(t_{n}\right)} \dot{y}_{t_{n}} .
\end{aligned} \\
& \text { Therefore } \tilde{w}=w\left(\cdot+t_{n}\right)-\frac{w\left(t_{n}\right)}{\dot{y}\left(t_{n}\right)} \dot{y}\left(\cdot+t_{n}\right) \text { on }[-1, \infty), \\
& \text { and boundedness of } \dot{y} \text { yields }\left|w\left(t^{\prime}\right)\right|+\infty \text { as } t^{\prime} \rightarrow \infty .
\end{aligned}
$$

For the application of Proposition 1 we need
PROPOSITION 2. Let $Y: \underline{R} \rightarrow \underline{R}$ be a solution of eq. $\left(g_{1}\right)$ with $Y_{0} \in N_{+}$and $\underline{P}\left(\mathrm{y}_{0}\right) \in \mathrm{s}$. Let $\mathrm{w}:[-1, \infty) \rightarrow \underline{\mathrm{R}}$ be a solution of $\dot{w}(t)=g_{j}^{\prime}(y(t-1)) w(t-1)$,

and let $c \in \underline{R}$, be given such that $w_{0}-c \dot{y}_{0}$ has no zero. Then there exists $t \geqq 0$, such that $w_{t}$ has no zero.

PROOF. $Y_{0} \epsilon N_{+}$and $\underline{P}\left(Y_{0}\right) \in S$ imply $|Y|<\frac{\gamma}{2}$ on $[-1, \infty)$ so that the coefficient of the linear variational equation along $y$ is bounded from below by $g_{1}\left(\frac{\gamma}{2}\right)>0$. It follows that the solution $w-c \dot{y}$ tends to $\infty$, or to $-\infty$, as $t \rightarrow \infty$. Note that $\dot{y}(t) \in g_{1}\left(\left[-\frac{\gamma}{2}, \frac{\gamma}{2}\right]\right)$ on $\underline{R}^{+}$. 
IV. HETEROCLINIC CONNECTIONS BETWEEN PERIODIC ORBITS,

HOMOCLINIC POINTS OF POINCARE MAPS, TRANSVERSALITY

Summary. The program sketched in Remark III.3.1 and in section III.4.1 is carried out. We choose an initial value $x$ on the upper branch of $U$ and a tangent vector $v \in \mathbb{T}_{\chi} U$. The nonlinearity $g_{1}$ is deformed outside the interval $[-\gamma, \gamma]$ to a one-parameter family of periodic nonlinearities $g_{b}, 4 \leq b \leq b^{*}$, all with a fixed period $\omega$, such that at a time $t_{+}>0$ the solutions $h_{b}$ of eq. $\left(g_{b}\right)$ through $x$ satisfy

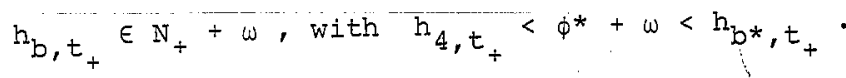

continuity and proposition III.3.1 then yield $b \in\left(4, b^{*}\right)$ with

(*) $\quad \underline{P}\left(h_{b, t_{+}}-\omega\right) \in S$.

In the construction of the functions $g_{b}$, care is taken that the solutions $w_{b}$ of the linear variational equation along $h_{b}$, with $w_{b},=v$, share a property which in case $(*)$ implies that

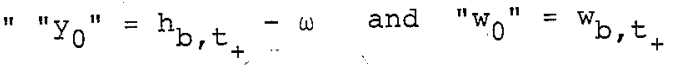

satisfy the condition of proposition III.4.1 for transversality.

For $g:=g_{b}$ with $(*)$ the modified return map $P^{\prime}$ is defined. $\chi$ becomes a transversal homoclinic point of $\mathrm{P}^{\prime}$, with respect to the hyperbolic fixed point $\phi^{*}$.

\section{ESCAPE FROM $x$ IN DOMAINS OF. MONOTONICITY}

It is convenient to begin with the additional assumption (A) $0<g$ : on $(\gamma, \infty)$.

we choose an initial value $x \in U$ above $S$ and a tängent vector $\mathrm{V} \in \mathrm{T}_{X} \mathrm{U} \subset \mathrm{H}$ according to

PROPOSITION 1. There exists $r \in\left(0, r_{2}\right)$ so that

$$
x:=\phi^{*}+r \lambda+u(r \lambda) \text { and } v:=\operatorname{Du}(r \lambda) \lambda+\lambda \epsilon^{*} \mathrm{~T}_{\chi} \mathrm{U}
$$

have the following properties.

(i) There are a trajectory $\left(x_{n}\right)_{-\infty}^{0}$ of $P$ in $U$ with $x_{0}=x$ and $x_{n} \rightarrow \phi^{*}$ as $n \rightarrow-\infty$, and a solution $h_{1}: \underline{R} \rightarrow \underline{R}$ of eq. $\left(g_{1}\right)$ with $h_{1, t_{n}}=x_{n}$ for all $n \in-\underline{N}_{0}$

where $\left(t_{n}\right)_{-\infty}^{0}$ in $\underline{R}^{-}$is given by $t_{0}=0$ and $t_{n-1}+\tau\left(x_{n-1}\right)=t_{n}$ for all $n \in-\underline{N}_{0}$. We have

(i.1) $\quad \dot{h}_{1}(0)<0$.

(ii) $h_{1}$ is $c^{2}$, and

(ii.1) $-\gamma<h_{1}$ on $\underline{R}$.

(ii.2) $\left|h_{1}\right|<\frac{\gamma}{2}$ on $(-\infty, 2]$.

(ii.3) $\mathrm{x}<\mathrm{h}_{1}$ on $[1,2]$.

There exists $t_{1}^{*}>2$, with $t_{1}^{*} \notin \underline{N}$ such that

(ii.4) $h_{1} \leqq h_{1}\left(t_{1}^{*}\right)$ on $\left(-\infty, t_{1}^{*}\right]$,

(ii.5) $\gamma+g_{1}(\gamma) \leqq h_{1}(t *)$,

(ii.6) $0<\dot{h}_{1}$ on $[t *, \infty)$.

(iii) The solution $w_{1}:[-1, \infty) \rightarrow \underline{R}$ of

$\dot{w}(t)=g_{1}^{\prime}\left(h_{1}(t-1)\right) w(t-1), w_{0}=v$

\section{satisfies}

(iii.1) $0<\dot{w}_{1}$ on $[1, \infty)$,

(iii.2) $0<\dot{w}_{1}$, on $[2, \infty)$.

COMMENT. " t去 $\notin \underline{\mathrm{N}} "$ in (ii) is technically convenient for proposition' 2 below. 

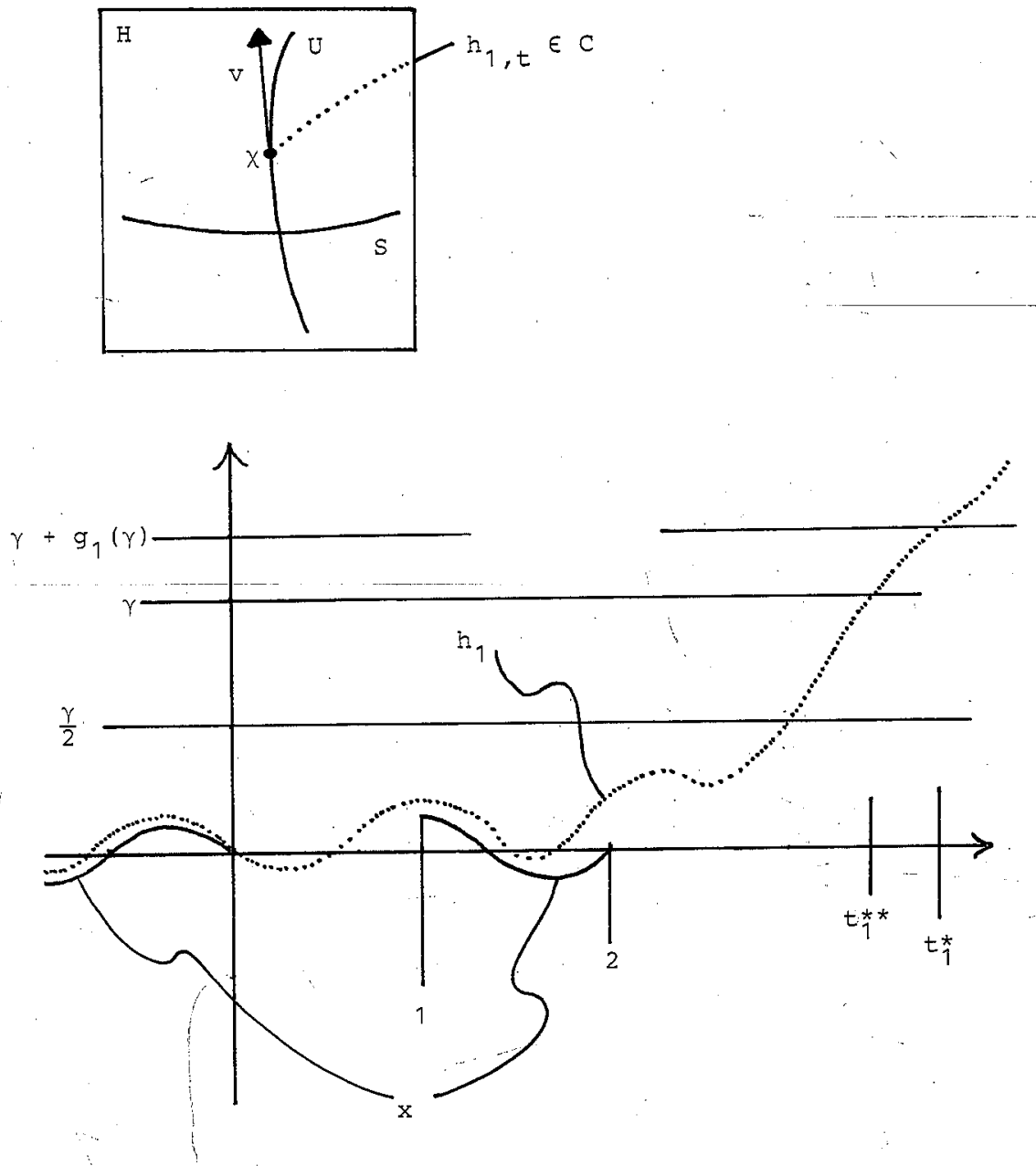

PROOF of Proposition 1. 1. For $0<r<r_{2}$, $r \lambda \in I_{r(u)}^{u *}$ (see Proposition III.2.1 (i')) so that $u(r \lambda)$ is defined. We have

$$
x_{r}^{*}:=\phi^{*}+r \lambda+u(r \lambda) \rightarrow \phi^{*} \text { as } r+0 \text {, }
$$

hence $g_{1}\left(x_{x}^{*}(-1)\right) \rightarrow g_{1}(x(-1))<0$.

Differentiation of the curve $\left(-r, r_{2}-r\right) \ni t \rightarrow x_{r+t}^{*} \in H$ at $t=0$ yields $v_{r}:=\operatorname{Du}(r \lambda) \lambda+\lambda \in \mathrm{T}_{\chi_{r}^{*}}^{\mathrm{U}}$. By $\operatorname{Du}(0)=0, \lim _{r \neq 0} \mathrm{v}_{r}=\lambda \neq 0$.

In view of Corollary III.1.2 (v) we choose $r \in\left(0, r_{2}\right)$ with

$$
g_{1}\left(x_{r}^{*}(-1)\right)<0 \text { and } D_{2} X_{1}\left(2, x_{r}^{*}\right) v_{r}>0 \text {. }
$$

and set $\chi:=\chi_{r}^{*}, \mathrm{v}:=v_{r}$.

2. $x_{0}:=x \in U$ extends to a (discrete) backward trajectory $\left(x_{n}\right)_{-\infty}^{0}$ of $P$ (Corollary II.1.1 (ii)). Using the solutions of eq. $\left(g_{1}\right)$ which

start at $x_{n}$ one can construct $h_{1}$ so that assertion (i) holds true (observe $\left.\dot{h}_{1}(0)=g_{1}\left(x_{r}^{*}(-1)\right)<0\right)$. We have

(1). $t_{n-1}+1<t_{n}<t_{n-1}+2$ for all $n \in-\underline{N}_{0}$.

3. As a solution on $\underline{R}, h_{1}$ is $C^{1}$. Eq. $\left(g_{1}\right)$ with $g_{1} C^{1}$ implies that $\dot{h}_{1}$ is also $C^{1}$ so that $h_{1}$ is $C^{2}$.

4. $h_{1, t_{n}}=x_{n} \in U \subset D \subset \phi^{*}+C_{r_{0}}$, the choice of $r_{0}$ in section I.7 and (1) yield

$$
\left|h_{1}\right|<\frac{\gamma}{2} \text { on }(-\infty, 2] \text {. }
$$

In adäition, $x_{2}<h_{1,2}$ because of $h_{1,0}=x_{0}=x_{r}^{*}, 0<x<r_{2}$ (see Proposition III.2.1 (ii.2)). Using Proposition I.7.1 (ii) we infer that there exists $t_{1}^{* *}>2$ with

$$
h_{1}\left(t_{1}^{*}\right)=\gamma \text { and }\left|h_{1}\right|<\gamma \text { on }\left[-1, t_{1}^{* *}\right) \text {. }
$$

5. We prove $\frac{\gamma}{2}<h_{1}$ on $\left[t_{1}^{*}, t_{1}^{* *}+1\right]$ : Set $t^{\prime}:=t_{1}^{* *}-2>0$. Consider restrictions of $h_{1}(2+\cdot)$ and $x(2+\cdot)$ to $[-1, \infty)$. Note (2) $\quad h_{1}\left(2+t^{\prime}\right)-x\left(2+t^{\prime}\right)=\gamma-x\left(2+t^{\prime}\right)>\gamma-\frac{\gamma}{4}=\frac{3 \gamma}{4}$. Proposition I.7.1 (i) is applicable, because of $x(2+\cdot)<h_{1}(2+\cdot)$ on $[-1,0],\left|h_{1}\right| \leqq \gamma$ on $\left(-\infty, t_{1}^{* *}\right],|x|<\frac{\gamma}{4}$. We get

$$
0<\dot{h}_{1}(2+\cdot)-\dot{x}(2+\cdot) \text { on }\left[t^{\prime}, t^{\prime}+1\right] \text {. }
$$

With (2),

$$
h_{1}(2+\cdot)-x(2+\cdot)>\frac{3 \gamma}{4} \text { on }\left[t^{\prime}, t^{\prime}+1\right] \text {, }
$$

i.e. for $t_{1}^{*} \leqq t \leqq t_{1}^{* *}+1, t-2 \in\left[t^{\prime}, t^{\prime}+1\right]$ and

$$
h_{1}(t)=h_{1}(2+t-2)>\frac{3 \gamma}{4}+x(2+t-2)>\frac{3 \gamma}{4}-\frac{\gamma}{4}=\frac{\gamma}{2} \text {. }
$$

6. By (A), $0<g_{1}(\gamma) \leqq g_{1}$ on $[\gamma, \infty)$. Using part 5 and eq. $\left(g_{1}\right)$, we obtain

$0<h_{1}$ on $\left[t_{1}^{* *}, \infty\right)$ and $0<g_{1}\left(\frac{\gamma}{2}\right) \leq \dot{h}_{1}$ on $\left[t_{1}^{* *}+1, \infty\right)$, hence $h_{1}(t) \rightarrow \infty$ as $t+\infty$. Now it is easy to find $t_{1}^{*}$ as desired. 
7. Prof of assertion (iii) : By the choice of $\mathrm{v}=\mathrm{v}_{\mathrm{r}}$. in part 1 ,

$\mathrm{w}_{1,2}=\mathrm{D}_{2} \mathrm{x}_{1}(2, x) \mathrm{v}>0$

so that the differential equation for $w_{1},(i i .1)$ and $0<g_{1}$ on $[-\gamma, \infty)$ yield $0<\dot{w}_{1}$ on $[2, \infty)$.

The first modification of $g_{1}$ outside the interval $[-\gamma, \gamma]$ is a deThe constant in some interval to the right of

$$
h_{1}\left(t_{1}^{*}+1\right) \quad>h_{1}\left(t_{1}^{*}\right)>y+g_{1}(\gamma) \text {. }
$$

amportant for transversality; it permits to obtain This step is already lmportant for trane verification of the condibasic relations (Proposition 2 below) for the

tion

$$
"\left|w_{0}-c \dot{y}_{0}\right|>0 "
$$

I Proposition ItI.4.2. (The application of Proposition 2 below follows in proposition 3.2 below. For more explanations, see Remark 3.1.)

Define $\gamma_{1}:=h_{1}\left(t_{1}^{*}+1\right)$. We have

$$
\gamma<\gamma+g_{1}(\gamma)<\gamma_{1}<h_{1}\left(\left[t_{1}^{*}+1\right]\right)<h_{1}\left(t_{1}^{*}+2\right)
$$

where $\left[t_{1}^{*}+1\right]$ is the integer in $\left(t_{1}^{*}+1, t_{1}^{*}+2\right)$.

$$
\text { Choose a } C^{1} \text {-function } g_{2}: \underline{R}+\underline{R} \text { with } g_{2}=g_{1} \text {. on }\left(-\infty, \gamma_{1}\right] \text {. }
$$$$
0<g_{2}^{\prime}(\xi) \text { for } \gamma_{1}<\xi<h_{1}\left(\left[t_{1}^{*}+1\right]\right) \text {, }
$$$$
0=g_{2}^{1}(\xi) \text { for } \quad h_{1}\left(\left[t_{1}^{*}+1\right]\right) \leqq \xi \text {. }
$$

clearly $0<g_{2}$ on $(0, \infty)$ and $0<g_{2}^{\prime}(\xi)$ for $-\gamma \leqq \xi<h_{1}\left(\left[t_{1}^{*}+1\right]\right)$.

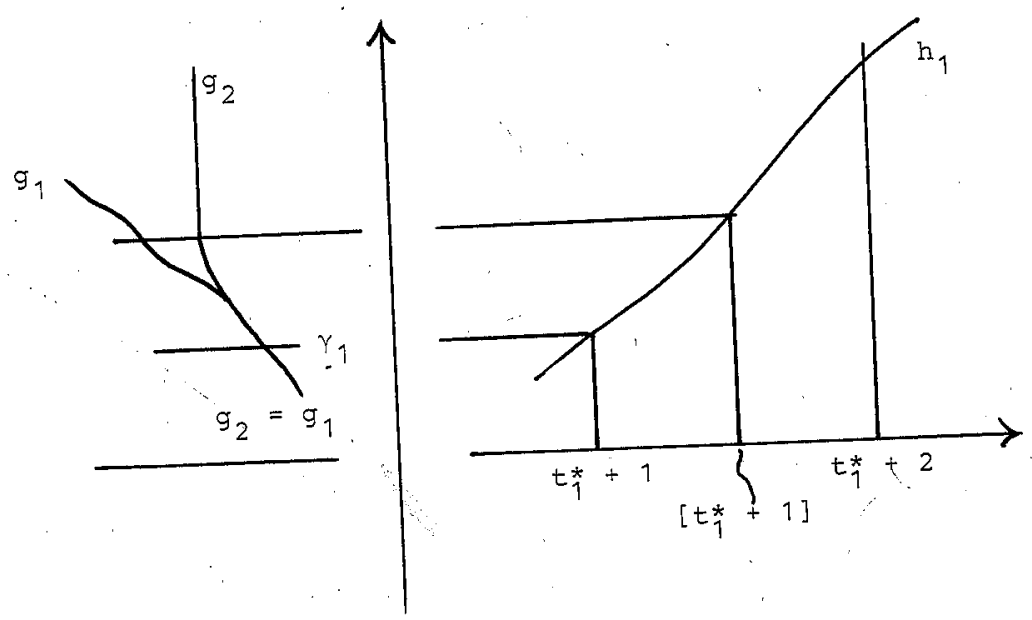

The restriction of $h_{1}$ to $\left(-\infty, t_{1}^{*} \div 2\right]$ satisfies eq. $\left(g_{2}\right)$ since $g_{2} \circ h_{1}=g_{1} \circ h_{1}$ on $\left(-\infty, t_{1}^{*}+1\right]$. It follows that there is a solution $h_{2}: \underline{R} \rightarrow \underline{R}$ of eq. $\left(g_{2}\right)$ which is $c^{2}$, with

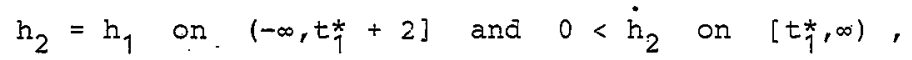

$\dot{h}_{2}(t)=g_{2}\left(h_{2}\left(\left[t_{1}^{*}+1\right]\right)\right)$ for all $t \geqq\left[t_{1}^{*}+1\right]+1$.

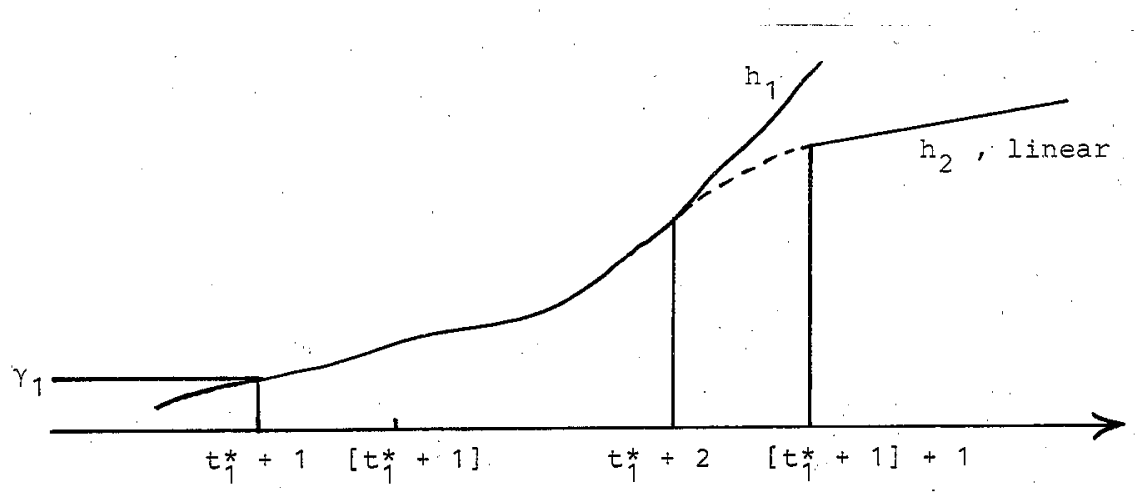

For the solution $w_{2}:[-1, \infty) \rightarrow \underline{R}$ of $\dot{w}(t)=g_{2}^{t}\left(h_{2}(t-1)\right) w(t-1), w_{0}=v$

we get

$$
\begin{aligned}
& w_{2}=w_{1} \text { on }\left[-1, t_{1}^{*}+2\right], \\
& 0<\dot{w}_{2}(t) \text { for } t_{1}^{*}+2 \leqq t<\left[t_{1}^{*}+1\right]+1, \\
& 0=\dot{w}_{2}(t) \text { for } \\
& \left.0<t_{1}^{*}+1\right]+1 \leqq t,
\end{aligned}
$$

PROPOSITION 2. With $C^{*}:=\frac{w_{2}\left(\left[t_{1}^{*}+1\right]+1\right)}{h_{2}\left(\left[t_{1}^{*}+1\right]+1\right)}>0$,

(i) $\quad w_{2}(t)=c^{*} \cdot \dot{h}_{2}(t)$ for all $t \geqq\left[t_{1}^{*}+1\right]+1$.

(ii) there exists $\varepsilon^{*}>0$ with

$w_{2}(t)-c^{*} \dot{h}_{2}(t) \neq 0$ for $\left[t_{1}^{*}+1\right]+1-\varepsilon^{*} \leqq t<\left[t_{1}^{*}+1\right]+1$.

PROOF. 1. Assertion (i) is obvious from the remarks above.

2. We show $0<g_{2}^{\prime}\left(h_{2}(t-1)\right)$ for all $t<\left[t_{1}^{*}+1\right]+1$ :

For $t \leqq t_{1}^{*}+2, h_{2}(t-1)=h_{1}(t-1) \leqq h_{1}\left(t t_{1}^{*}+1\right)$, hence 
The next step is a deformation of $g_{2}$ to an increasing function on an

$h_{2}(t-1) \in\left[-\gamma, h_{1}\left(t t_{1}^{*}+1\right)\right]$

so that

$$
g_{2}^{2}\left(h_{2}(t-1)\right)>0 \text {. }
$$

If $t_{1}^{*}+2<t<,\left[t_{1}^{*}+1\right]+1$ then

$$
\begin{aligned}
& -\gamma<h_{1}\left(t_{1}^{*}+1\right)=h_{2}\left(t_{1}^{*}+1\right)<h_{2}(t-1)<h_{2}\left(\left[t_{1}^{*}+1\right]\right) \\
& =h_{1}\left(\left[t_{1}^{*}+1\right]\right),
\end{aligned}
$$

and the choice of $g_{2}$ yields $0<g_{2}^{t}\left(h_{2}(t-1)\right)$.

$$
\begin{aligned}
& \text { 3. By } w_{2,0}=v \in H, w_{2}(0)=0 \text {. Hence } \\
& \dot{w}_{2}(1)=g_{2}^{\prime}(\ldots) w_{2}(0)=0>c^{*} g_{2}^{\prime}\left(h_{2}(0)\right) \dot{h}_{1}(0) \quad \text { (part 2, Proposi- } \\
& \text { tion 1. (i.1)) } \\
& =c^{*} g_{2}^{1}\left(h_{2}(0)\right) \dot{h}_{2}(0)=c^{*} \ddot{h}_{2}(1),
\end{aligned}
$$

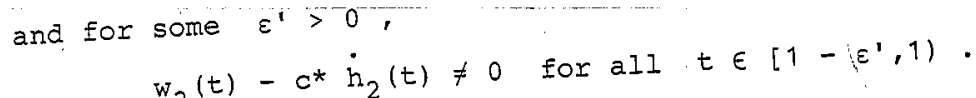

$w_{2}(t)-c^{*} h_{2}(t) \neq 0$ for all $t \in\left(1-e^{\prime}, 1\right)$ smallest $n$ in

4. Suppose assertion (ii) is false. Then there ${ }$ a smats $t_{\varepsilon}$ in $\left\{1, \ldots,\left[t_{1}^{*}+1\right]+1\right\}$ such that for every $\varepsilon>0$, thensider $(n-\varepsilon, n)$ with $w_{2}\left(t_{\varepsilon}\right)-c^{*} \dot{h}_{2}\left(t_{\varepsilon}\right)=0$. By part $3,2 \leqq n$. Consider $n-1$. For some $\varepsilon^{\prime \prime}>0$,

$w_{2}(t)-c^{*} \dot{h}_{2}(t) \neq 0$ for all $t \in\left[n-1-\varepsilon^{n}, n-1\right)$. Using part 2 we infer

$\dot{w}_{2}(t)-c^{*} \ddot{h}_{2}(t)=g_{2}^{\prime}\left(h_{2}(t-1)\right)\left\{w_{2}(t-1)-c^{*} \dot{h}_{2}(t-1)\right\} \neq 0$ for $n-\varepsilon " \leqq t<n$, which implies a contradiction to the properties of n.

The case $\mathrm{w}_{2}>\mathrm{c}^{*} \dot{\mathrm{h}}_{2}$ in Proposition $2(i \mathrm{i})$ :

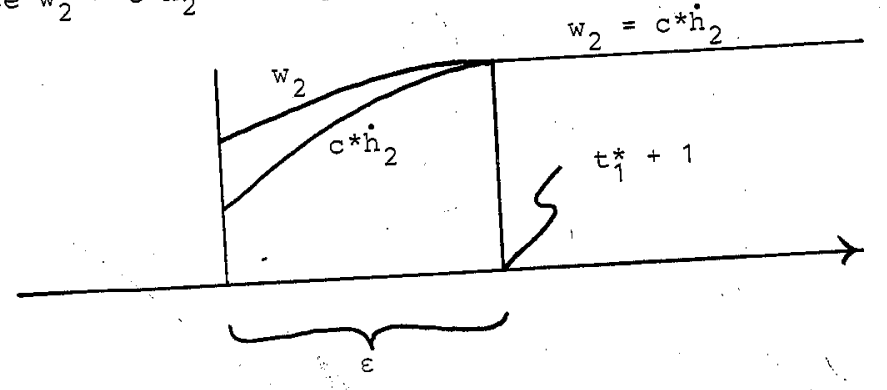

interval with left endpoint inside the interval

$$
\left(h_{2}\left(\left[t_{1}^{*}+1\right]\right), h_{2}\left(\left[t_{1}^{*}+1\right]+1\right)\right) \text {. }
$$

This is necessary for transversality:

Suppose $g$ is a (periodic) continuation of the restriction of $g_{2}$ to the interval $\left[-\gamma_{1} h_{2}\left(\left[t_{1}^{*}+1\right]+1\right)\right]$. For a solution $h$ of eq. $(g)$ with $h_{0}=x$ and for the solution $w$ of the linear variational equation along $h$ with $w_{0}=v$, we infer

$$
\begin{aligned}
& w(t)=w_{2}(t)=c^{*} \dot{h}_{2}(t)=c^{*} \dot{h}(t) \\
& \text { for }\left[t_{1}^{*}+1\right]+1 \leqq t \leqq\left[t_{1}^{*}+1\right]+2,
\end{aligned}
$$

since $g_{2}^{\prime}(\xi)=0$ for $h_{2}\left(\left[t_{1}^{*}+1\right]\right) \leqq \xi \leqq h_{2}\left(\left[t_{1}^{*}+1\right]+1\right)$. Thereby $w_{t}=c^{*} \dot{h}_{t}$ for all $t \geq\left[t_{1}^{*}+1\right]+2$.

For the modified Poincare'map $P^{\prime}$ (see Remark III.3.1 and Section III.4), $D^{\prime}(x) v$ will be given by the projection of some $w_{t} \in C$,

$t \geq\left[t_{1}^{*}+1\right]+2$, onto $H$, parallel to $\dot{h}_{t}$ - the result is

$$
0 \in T_{P^{\prime}(x)} S \text {. }
$$

Set $t_{3}^{*}:=\left[t_{1}^{*}+1\right]+1$ so that

$$
t_{1}^{*}+2<t_{3}^{*} \text {. }
$$

Choose $t_{2}^{*}<t_{3}^{*}$ so close to $t_{3}^{*}$ that

$$
t_{3}^{*}+\theta<t_{2}^{*} \text { and } t_{3}^{*}-\varepsilon^{*}<t_{2}^{*} .
$$

Clearly

$$
t_{1}^{*}<t_{3}^{*}-2<t_{3}^{*}-1<t_{\frac{\pi}{3}}^{*}-\frac{1}{3}<t_{3}^{*}+\theta \leqq \max \left\{t \frac{*}{3}+\theta, t_{3}^{*}-\varepsilon^{*}\right\}
$$

$<t_{2}^{*}<t_{3}^{*}$.

Set $\gamma_{2}:=h_{2}\left(t_{2}^{*}\right)>\gamma_{1}$ and choose a $C^{1}$-function $g_{3}: \underline{R} \rightarrow R_{-}$with $g_{3}=g_{2}$ on $\left(-\infty, \gamma_{2}\right]$,

$0<g_{3}^{\prime}$ on $\left(\gamma_{2}, \infty\right)$.

The properties of $h_{2}$, in particular

$$
-\gamma<h_{2}(t-1) \leqq h_{2}\left(t \frac{*}{2}\right)=\gamma_{2} \text { for } t \leqq t_{2}^{*}+1
$$

and

$$
\dot{h}_{2}(t)=g_{2}\left(h_{2}(t-1)\right)=g_{3}\left(h_{2}(t-1)\right) \text { for } t \leqq t_{2}^{*}+1 \text {, }
$$

imply that there is a $c^{2}$-solution $h_{3}: \underline{R} \rightarrow \underline{R}$ of eq. $\left(g_{3}\right)$ with 
Set $\gamma_{3}:=h_{3}\left(t_{3}^{*}\right) \geq \gamma_{2}$.

REMARK 1 - the basic observation, for the search of a periodic continuation $g$ of the restriction of $g_{3}$ to the interval $\left[-\gamma, \gamma_{3}\right]$ such that there is a heteroclinic solution $h$ of eq, ( $g$ ) from $x$ to another periodic solu-

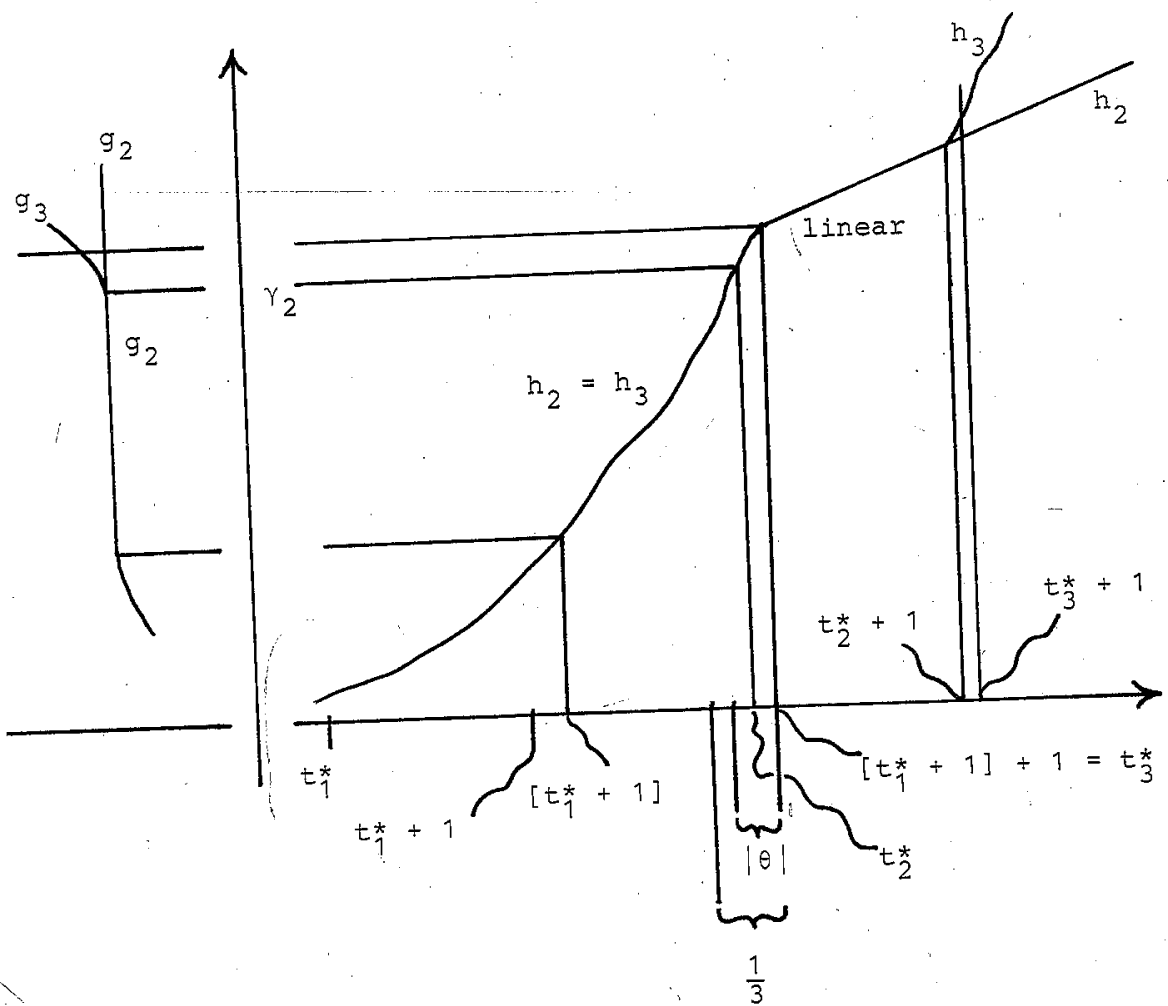
tion:

The delay in the differential equation implies that for any continuation $g$ of $g_{3} \mid\left[-\gamma, \gamma_{3}\right]$, the solution $h:[-1, \infty) \rightarrow \underline{R}$ with $h_{0}=x$ coincides with $h_{3}$ not only on $\left[-1, t_{3}^{*}\right]$ but also on the longer interval $\left[-1, t_{3}^{*}+1\right]$. This property allows to choose $g$ on intervals

$$
h_{3}(I)=\left(\gamma_{3}, h_{3}\left(t_{3}^{*}+1\right) \text { with } I \subset\left(t_{3}^{*}, t_{3}^{*}+1\right)\right.
$$

so that eq. $(g)$ steers $\dot{h}(t), t \in I+1$, close to prescribed values.

Two choices of g outside $\left[-\gamma, \gamma_{3}\right]$ :

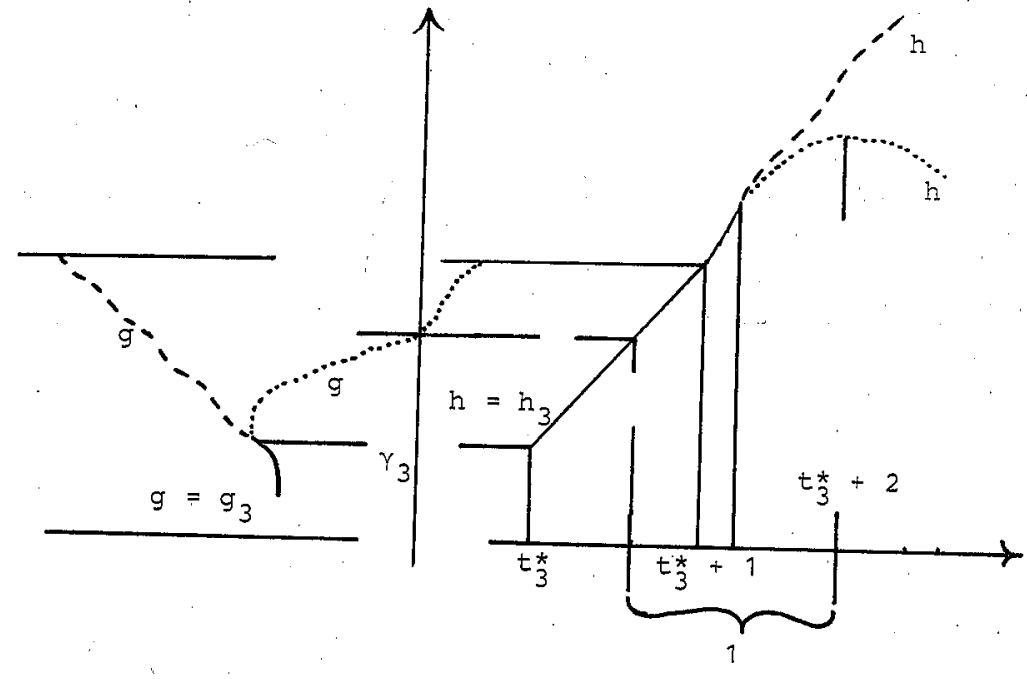


whali steer $\dot{h}$ close to $\dot{\phi}^{* *}\left(\phi^{* *} \in \mathbb{N}_{+}\right.$was not far from $\phi^{*}$

section III.3), and we shall find $t_{+} \approx t_{2}^{*}+2$ with

$$
h_{t_{+}} \in N_{+}^{+} w
$$

where $w$ is the minimal period of $g$

the construction of nonlinearities until now and

The subtleties in the construction of non functions, furtheron are mainly caused by the objective of "sine-like" function. with not too many zeros per period, and by the ain

We $f_{i x}$ some $t_{4}^{*}>t_{3}^{*}$ so close to $t_{3}^{*}$ that

$$
t_{3}^{*}+\theta<t_{4}^{*}+\theta<t_{2}^{*}<t_{3}^{*} .
$$

ancreasing finite sequence of points $\gamma_{n}>\gamma_{3}$ :

$$
\begin{aligned}
& \gamma_{4}:=h_{3}\left(t_{4}^{*}\right), \\
& \gamma_{z}:=h_{3}\left(t_{z}\right) \text { where } t_{z}:=t_{4}^{*}+\frac{2}{3} .
\end{aligned}
$$

uniqu zero strictly between 0 and the minimal period of $z$ we are going to construct.

the nonlinearities where $t_{5}^{*}:=t_{4}^{*}+1+\theta>t_{z}$,

$$
\begin{aligned}
& \gamma_{5}:=h_{3}\left(t_{5}^{*}\right) \text { where } t_{6}^{*}:=t_{2}^{*}+1>t_{5}^{*} \text {, } \\
& \gamma_{6}:=h_{3}\left(t_{7}^{*}\right) \text { where } t_{7}^{*}:=t_{3}^{*}+1>t_{6}^{*} \text {, } \\
& \gamma_{8}:=h_{3}\left(t_{8}^{*}\right) \text { where } t_{8}^{*}:=t_{4}^{*}+1>t_{7}^{*} \text {. }
\end{aligned}
$$

inal period of the nonlinearities to be con$\omega:=\gamma_{8}+\gamma$

set $t_{+}:=t_{4}^{*}+2=t_{8}^{*}+1$. We shall have

$$
h_{t_{+}} \in \phi^{*}+c_{2 r_{3}}+w \in \mathbb{N}_{+}+w
$$

for solutions $h$ with $h_{0}=x \in U$.

Define

$$
\begin{aligned}
& g_{4}(\xi):=g_{3}(\xi) \text { for }-\gamma \leq \xi \leqq \gamma_{3}, \\
& g_{4}(\xi):=g_{3}(\xi-\omega) \text { for } \omega-\gamma=\gamma_{8} \leqq \xi \leqq \gamma_{8}+2 \gamma=\omega+\gamma .
\end{aligned}
$$
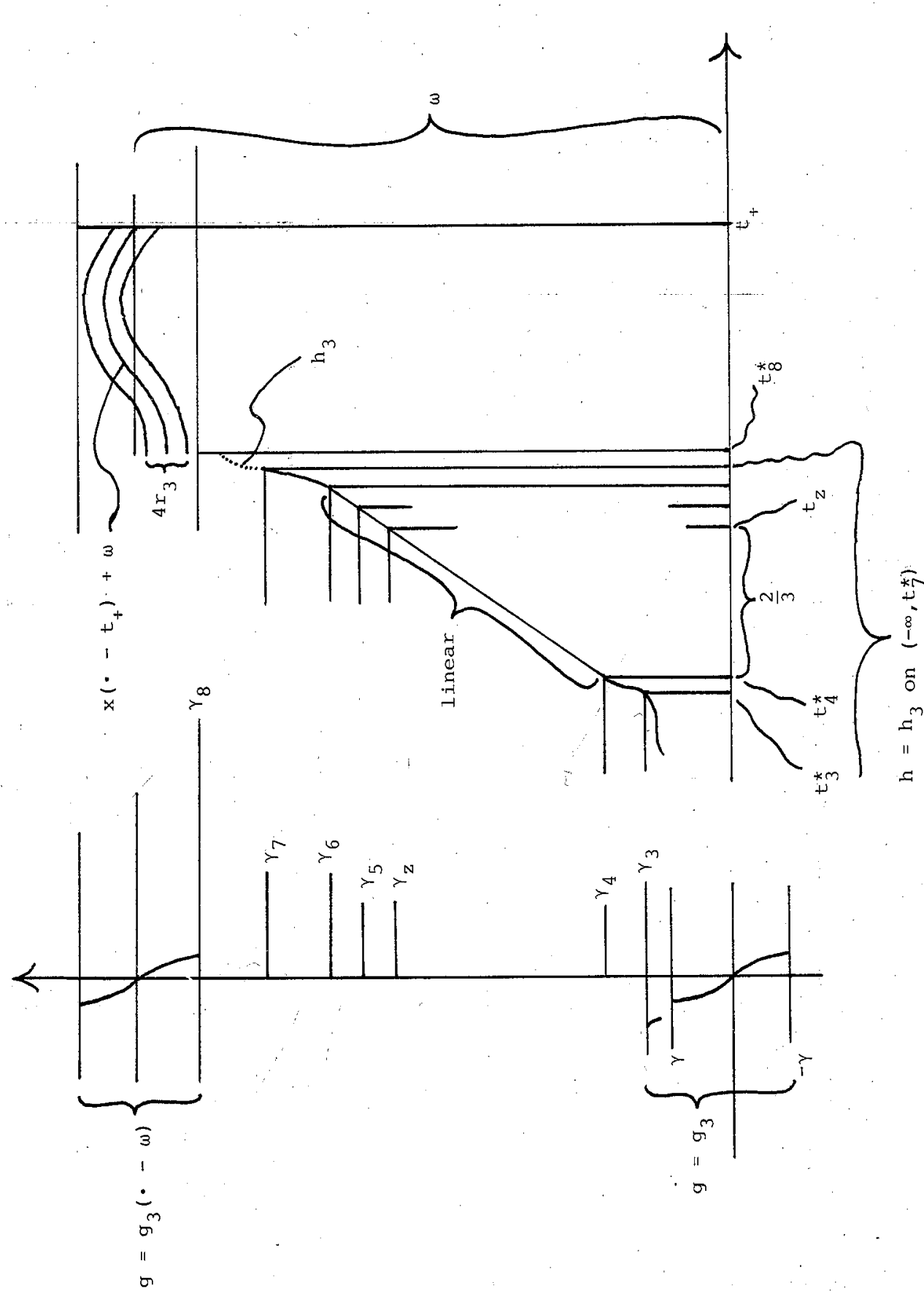
The properties of $\phi^{* *}$ (Proposition III.3.2) imply

$$
\begin{aligned}
& 0<\dot{\phi}^{* *}\left(t-\left(t_{4}^{*}+1\right)\right) \text { for } t_{4}^{*} \leqq t<t_{4}^{*}+\frac{2}{3}=t_{z}, \\
& \dot{\phi}^{* *}\left(t-\left(t_{4}^{*}+1\right)\right)<0 \text { for } t_{z}<t \leqq t_{4}^{*}+1=t_{8}^{*} .
\end{aligned}
$$

$\left.z<t^{*}+1+\theta\right]$ strictly increaRecall that $h_{3}$ maps the interval $\left[t_{4}^{*}, t_{4}^{*}+1+\theta\right]$ strictly increasing onto the interval $\left[\gamma_{4}, \gamma_{5}\right]$.

It follows that we can define $g_{4}(\xi)$ for $\gamma_{4} \leqq \xi \leqq \gamma_{5}$ by a $c^{1}$-function such that

$$
\begin{aligned}
& \text { (1) }\left|g_{4}\left(h_{3}(t)\right)-\dot{\phi}^{* *}\left(t-\left(t_{4}^{*}+1\right)\right)\right|<r_{4} \\
& \text { for all } t \in\left[t_{4}^{*}, t_{4}^{*}+1+\theta\right]=\left[t_{4}^{*}, t_{5}^{*}\right] \text {, } \\
& 0<g_{4} \text { on }\left[\gamma_{4}, \gamma_{z}\right) \text { and } g_{4}<0 \text { on }\left(\gamma_{z}, \gamma_{5}\right] \text {. }
\end{aligned}
$$

(Note that we can not simply set

$$
g_{4}(\xi):=\phi^{* *}\left(\left(h_{3} \mid[\ldots]\right)^{-1}(\xi)-\left(t_{4}^{*}+1\right)\right)
$$

since $\dot{\phi}^{* *}$ is not differentiable at $t_{*} \in\left(-1,-\frac{2}{3}\right)$ : $\left.\lim _{t \uparrow t_{*}} \ddot{\phi}^{* *}(t)=0 \neq g_{1}^{\prime}\left(x\left(t_{*}-1\right)\right) \dot{x}\left(t_{*}-1\right)=\ddot{x}\left(t_{*}\right)=\lim _{t \psi t_{*}} \ddot{\phi}^{* *}(t).\right)$

REMARR 2. The purpose of (1) is to obtain $\dot{h}_{4}\left(t_{+}+\cdot\right) \approx \dot{\phi}^{* *}$ on $[-1, \theta]$ for the solution $h_{4}$ of eq. $\left(g_{4}\right)$, with $h_{4}=h_{3}$ on $\left(-\infty, t_{3}^{*}+1\right]-c o m-$ pare Remark 1.

COROLIARY 1. (i) $\left(g_{4}\left(\gamma_{8}\right)<g_{4}(\xi)\right.$ for $\gamma_{z} \leqq \xi \leqq \gamma_{5}$.

(ii) For $\gamma_{4} \leqq \xi \leqq \gamma_{2}$ and $\gamma \leqq \xi^{\prime} \leqq \gamma_{3}, g_{4}(\xi)<g_{4}(\gamma) \leqq g_{4}\left(\xi^{\prime}\right)$.

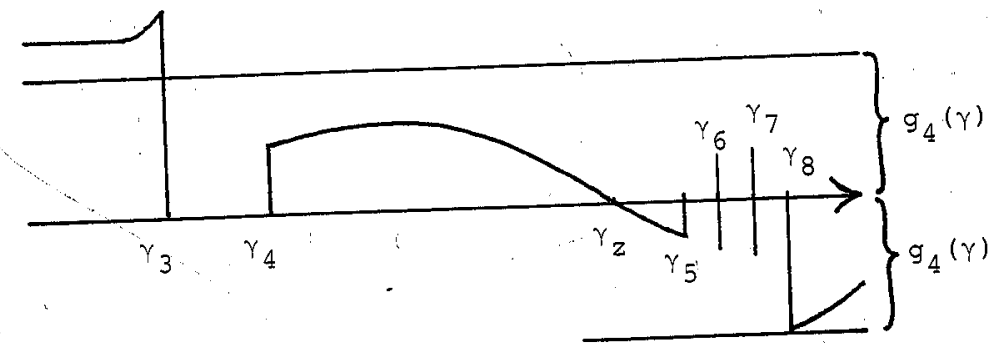

PROOF of Corollary 1. For $\gamma_{z} \leqq \xi \leqq \gamma_{5}$,

$g_{4}(\xi)>\min \dot{\phi} * *-r_{4} \geq \min \dot{x}-r_{4} \geqq g_{1}\left(-\frac{\gamma}{4}\right)-r_{4}>g_{1}(-\gamma)$

$$
=g_{4}\left(\gamma_{8}\right)
$$

(by (III.3.4)), and for $\gamma_{4} \leqq \gamma \leqq \gamma_{z}$,

$$
g_{4}(\xi)<\max \dot{\phi}^{* *}+r_{4} \leqq \max \dot{x}+r_{4} \leqq g_{1}\left(\frac{\gamma}{4}\right)+r_{4}<g_{1}(\gamma)=\dot{g}_{4}(\gamma) .
$$

Use $0 \leqq g_{3}^{\prime}=g_{4}^{\prime}$ on $\left[\gamma, \gamma_{3}\right]$.

REMARK 3. The gap between $\gamma_{3}$ and $\gamma_{4}$ will be closed in such a way that $h_{4}$ (see Remark 2) reaches the value $\phi^{* *}(-1) \div \omega-r_{3}$ at $t=t_{8}^{*}$. Observe that $h_{4}$ will then satisfy

$$
h_{4}\left(t_{8}^{*}\right)=h_{4}\left(t_{7}^{*}\right)+\int_{t_{7}^{*}}^{t_{8}^{*}} \dot{h}_{4}=r_{7}+\int_{t_{3}^{*}}^{t_{4}^{*}} g_{4} \circ h_{4}=r_{7}+\int_{t_{3}^{*}}^{t_{3}^{*}} g_{4} \circ h_{3} .
$$

$h_{3}$ maps the interval $\left[t_{3}^{*}, t_{4}^{*}\right]$ strictly increasing onto the interval $\left[\gamma_{3}, \gamma_{4}\right]$, and

$$
\gamma_{7}<\gamma_{8}<-\frac{\gamma}{4}+\gamma_{8}+\gamma-2 r_{3}<\phi * *(-1)+\omega-r_{3},
$$

by $|x|<\frac{\gamma}{4}$, by the choice of $r_{3}$ in Section III. 3 and by Proposition III. 3.2 (i).

It follows that we can define $g_{4}$ on $\left(\gamma_{3}, \gamma_{4}\right)$ such that $g_{4} \mid\left[-\gamma_{1}, \gamma_{5}\right]$ is $C^{1}$, with

$$
g_{4}(\xi)>0 \text { for } \gamma_{3}<\gamma<\gamma_{4}
$$

(2) $\quad r_{7}+\int_{t_{3}^{*}}^{t_{4}^{*}} g_{4} \circ h_{3}=\phi^{* *}(-1)+\omega-r_{3}$. 

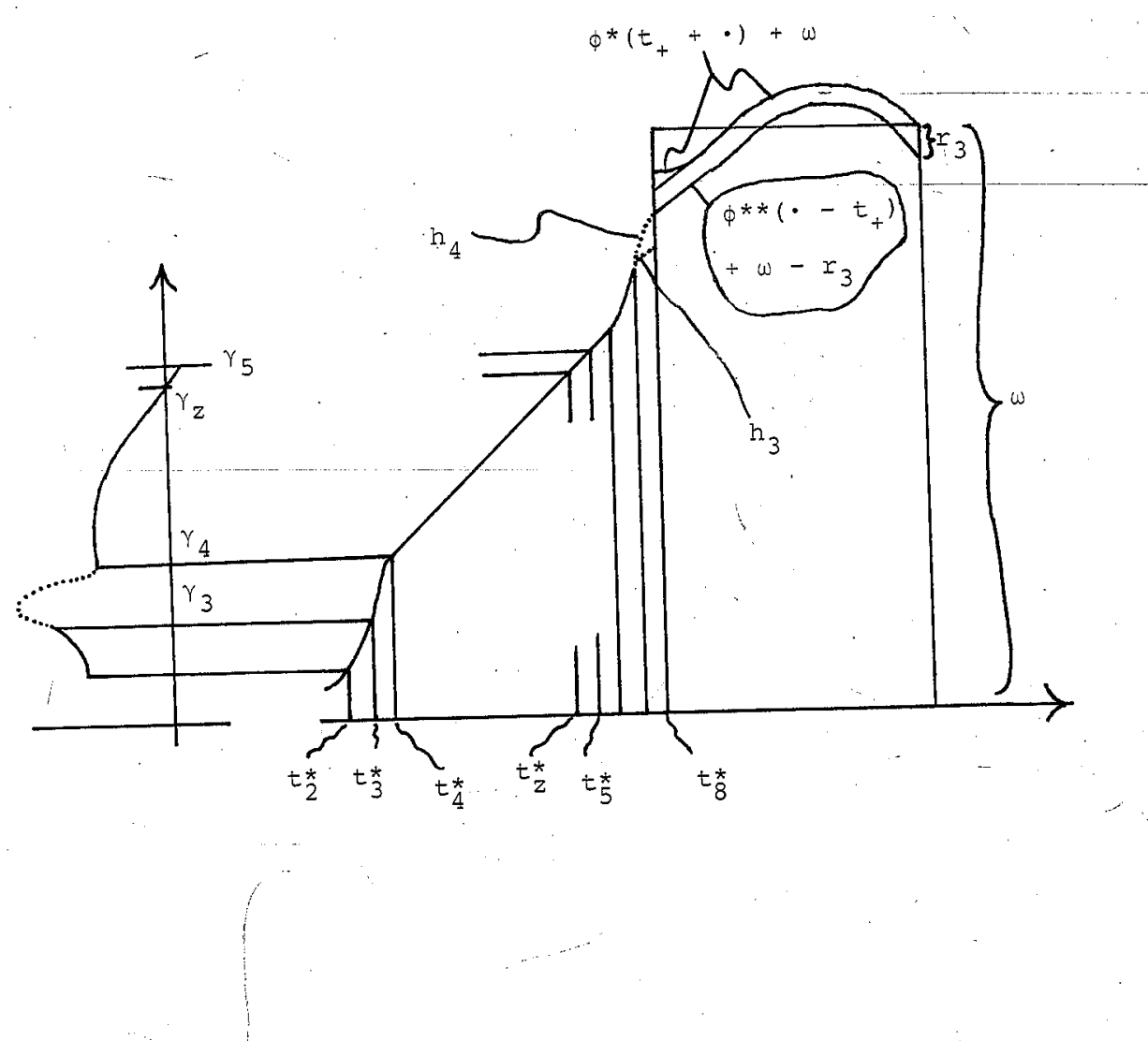

REMARK 4. (1) and (2) together will result in

$$
h_{4}\left(t_{+}+\cdot\right) \approx \phi^{* *}+w-r_{3} \text { on }[-1, \theta],
$$

compare Remarks 2 and 3 .

Next, the gap between $\gamma_{5}$ and $\gamma_{8}$ is closed in such a way that

$g_{4} \mid[-\gamma, \omega+\gamma]$ is $c^{1}$, and

(3) $c_{1}<g_{4}(\xi)<0$ for $\gamma_{z}<\xi \leqq \gamma_{8}$,

which is possible because of $. c_{1}<g_{1}(-\gamma)$, see section III.3, and corollary 1 (i), and
(4)
$0<g_{4}^{\prime}(\xi)$
for $\gamma_{6} \leqq \xi<\gamma_{8}$.

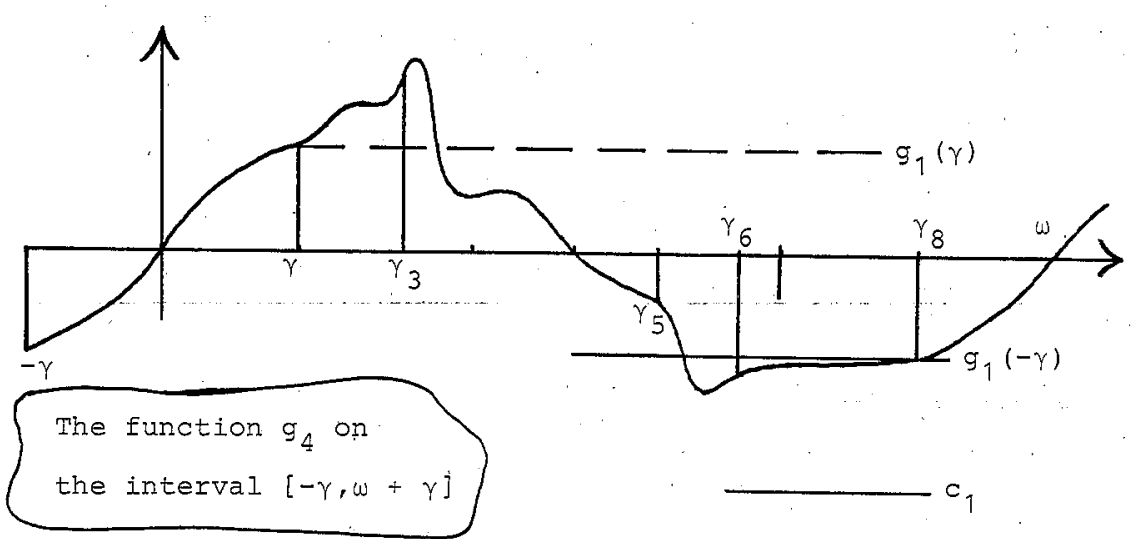

REMARK 5. Condition (3) will imply $c_{1}<\dot{h}_{4}\left(t_{+}+\cdot\right)$ on $[\theta, 0]$ so that one can use Proposition III.3.3 and extend

$$
h_{4}\left(t_{+}+\cdot\right) \approx \phi^{* *}+\omega-r_{3}
$$

from the interval $[-1, \theta]$ to all of $[-1,0]$.

condition (4) is important for transversality.

Finally, the w-periodic continuation $g_{4}$ of the function constructed so far is embedded into a family of w-periodic $C^{1}$-functions $g_{b}: \underline{R} \rightarrow \underline{R}$, $b \geqq 4$, such that solutions $h_{b}$ of eq. $\left(g_{b}\right)$ with $h_{b}=h_{3}$ on $\left(-\infty, t \frac{*}{7}\right)$ will have values $h_{b}(t *)$ which increase from

$$
h_{4}\left(t_{8}^{*}\right)=\phi^{* *}(-1)+\omega-r_{3}
$$

to $\infty$ as $b \rightarrow \infty$ :

We choose an w-periodic $C^{1}$-function $g^{*}: \underline{R} \rightarrow \underline{R}$ with

$$
g^{*}=0 \text { on }\left[0, \gamma_{3}\right] \cup\left[\gamma_{4}, \omega\right] \text { and } 0<g^{*} \text { on }\left(\gamma_{3}, \gamma_{4}\right) \text {, }
$$
and set

$$
g_{b}:=g_{4}+(b-4) g^{*} \text { for all } b \geqq 4 .
$$

COROLLARY 2. (i) FOr all b $\geqq 4$,

$$
g_{b}=g_{3} \text { on }\left[-\gamma, \gamma_{3}\right] \text { and } g_{b}=g_{4} \text { on }\left[\gamma_{4}, \omega+\gamma\right] \text {, }
$$$$
0<g_{b} \text { on }\left(0, \gamma_{z}\right) \text { and } g_{b}<0 \text { on }\left(\gamma_{z}, \omega\right) \text {. }
$$ 
$\phi * *(-1)+w-r_{3} \leqq \gamma_{7}+\int_{t_{3}^{*}}^{t_{4}^{*}} g_{b} \circ h_{3} \leqq \phi^{* *}(-1)+\omega+r_{3}$

for all $b \in\left[4, b^{*}\right]$, and

$\gamma_{7}+\int_{t_{3}^{*}}^{t_{4}^{*}} g_{b *} \circ h_{3}=\phi * *(-1)+\omega+r_{3}$.

PROOF of assertion (ii). On the interval $\left[t_{3}^{*}, t_{4}^{*}\right]$

$g_{b} \circ h_{3}=g_{4} \circ h_{3}+(b-4) g * \circ h_{3}$.

Recall (2) and use

$$
\int_{t_{3}^{*}}^{t_{4}^{*}} g^{*} \circ h_{3} \geq \frac{\int_{t_{3}^{*}}^{t_{4}^{*}} g^{*} \circ h_{3} \cdot \dot{h}_{3}}{\max _{\left[t_{3}^{*}, t_{4}^{*}\right]} \dot{h}_{3}}=\frac{\int_{\gamma_{3}}^{\gamma} g^{*}}{\max _{\left[t_{3}^{*}, t_{4}^{*}\right]}^{\dot{h}_{3}}}>0 \cdot
$$

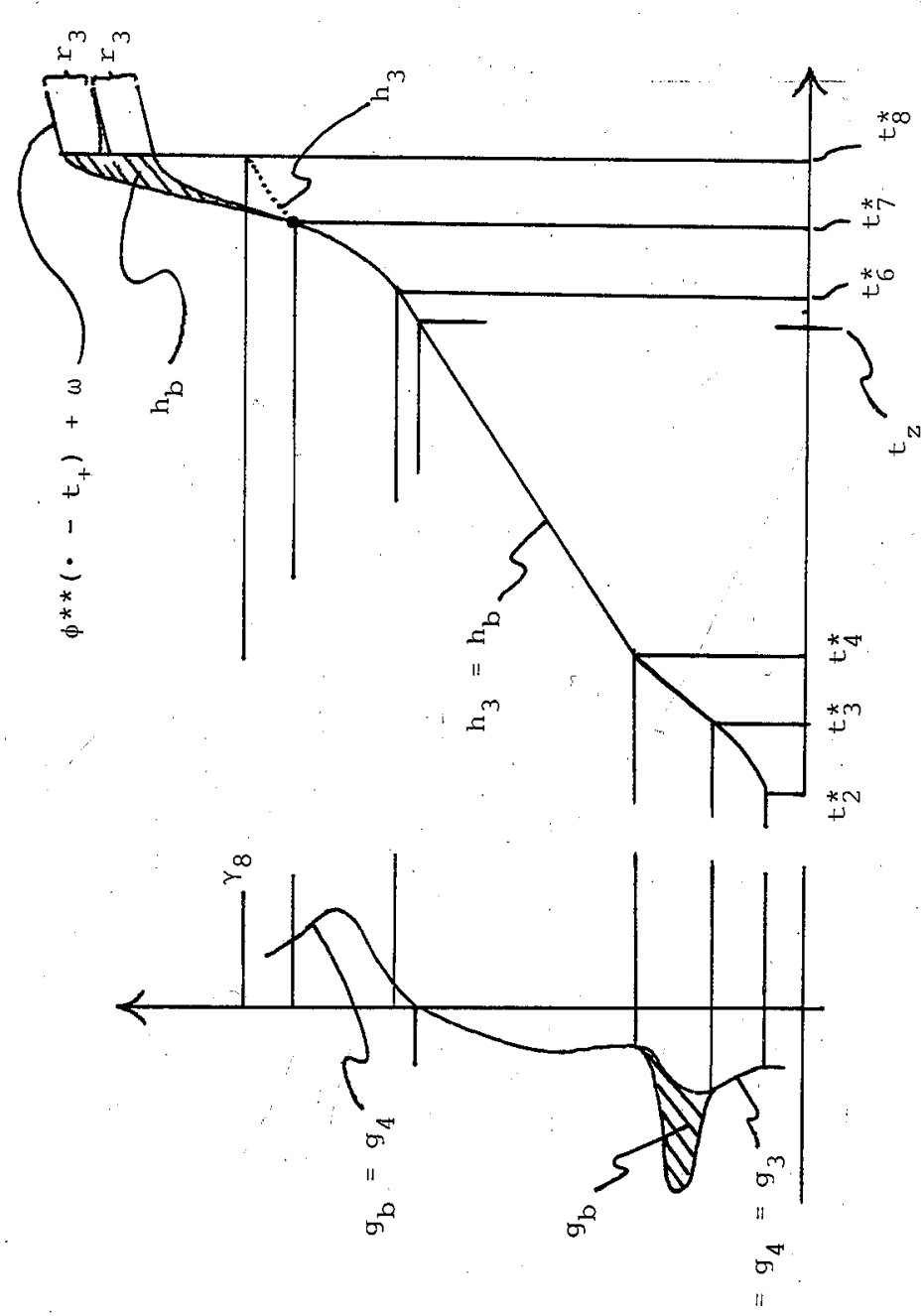




$$
=\gamma_{7}+\int_{t_{3}^{*}}^{t \frac{*}{*}} g_{b} \circ h_{3}+\int_{t_{4}^{*}}^{t * t}+1+t g_{4}^{*} \circ h_{3} .
$$

3. TRANSPORT OF $x \in U$ AND $v \in T_{X} U$ FOR $0 \leqq t \leqq t_{+}$

PROPOSIIION 1. (i) Let $b \geqq 4$. There is a solution $h_{b}: \underline{R} \rightarrow \underline{R}$ of eq. $\left(g_{b}\right)$

with $h_{b}^{-}=h_{3}$ on $\left(-\infty, t_{7}^{*}\right] \quad\left(\right.$ and $\left.h_{b, 0}=x \in U\right)$, and with

$0<\dot{h}_{b}$ and $\gamma_{z}<h_{b}$ on $\left(t_{z}, t_{8}^{*}\right]$.

For $4 \leqq b \leqq b^{*}, h_{b}<\omega$ on $\left[t_{z}, t_{8}^{*}\right]$ and

$\mathrm{h}_{\mathrm{b}, \mathrm{t}_{+}}-\omega \in \phi^{*}+\mathrm{C}_{2 \mathrm{r}_{3}} \subset \mathrm{N}_{+} \cdot$

(iii) $h_{4, t_{+}}-w<\phi^{*}<h_{b^{*}, t_{+}}-w$.

PROOF. 1. Let $b \geqq 4$. FOr $t \leqq t_{7}^{*}=t_{3}^{*}+1$,

$$
-\gamma<h_{3}(t-1) \leqq h_{3}\left(t \frac{\pi}{3}\right)=\gamma_{3},
$$

see (1.3). With $g_{b}=g_{3}$ on $\left[-\gamma, \gamma_{3}\right]$.

$$
\dot{h}_{3}(t)=g_{3}\left(h_{3}(t-1)\right)=g_{b}\left(h_{3}(t-1)\right) \text {. }
$$

This yields existence of $h_{b}$ with $h_{b}=h_{3}$ on $\left(-\infty, t_{7}^{*}\right]$. We have

$$
\begin{aligned}
& h_{b}\left(t_{z}\right)=h_{3}\left(t_{z}\right)=\gamma_{z}, \\
& \dot{h}_{b}(t)=\dot{h}_{3}(t)>0 \text { for } t_{z}<t \leq t_{7}^{*},
\end{aligned}
$$

see (1.3). Let $t_{7}^{*}<t \leqq t_{8}^{*}$. Then $t_{3}^{*}<t-1 \leq t_{4}^{*}$, and

$$
\begin{aligned}
& h_{b}(t-1)=h_{3}(t-1) \in\left(\gamma_{3}, \gamma_{4}\right) \\
& \dot{h}_{b}(t)=g_{b}\left(h_{b}(t-1)\right)>0 .
\end{aligned}
$$

Consequently, $\gamma_{z}<h_{b}$ on $\left[t_{z}, t_{8}^{*}\right]$

2. Let $4 \leqq b \leqq b^{*}$. For $t_{z} \leqq t \leqq t_{8}^{*}$

$$
h_{b}(t) \leqq h_{b}\left(t_{8}^{*}\right)=h_{b}\left(t_{7}^{*}\right)+\int_{t_{7}^{*}}^{t \frac{*}{8}} \dot{h}_{b}=h_{3}\left(t_{7}^{*}\right)+\int_{t_{3}^{*}}^{t_{4}^{*}} g_{b} \circ h_{b}
$$$$
=\gamma_{7}+\int_{t_{3}^{*}}^{t_{4}^{*}} g_{b} \circ h_{3} \leqq \phi^{* *}(-1)+\omega+r_{3}
$$

(by Corollary 2.2 (ii))

$\leq \phi^{*}(-1)+\omega+r_{3}<\omega$.

(by Proposition III.3.2 (iii) and by (III.3.2)).

3. Let $-1 \leqq t \leqq \theta$. With eq. $\left(g_{b}\right)$ and Corollary 2,2 (i), we get

$$
h_{b}\left(t_{+}+t\right)=h_{b}\left(t_{7}^{*}\right)+\int_{t_{\frac{*}{3}}^{*}}^{t_{4}^{*}+1+t} g_{b} \circ h_{b}
$$

IOn: $\left(-\infty, t_{7}^{*}\right], h_{b}=h_{3} \cdot h_{3}$ maps $\left[t_{4}^{*}, t_{7}^{*}\right]$ onto $\left[\gamma_{4}, \gamma_{7}\right]$ where $g_{b}=$ $g_{4}$. We have

$$
\left.t_{4}^{*}+1+t=t_{8}^{*}+t \leqq t_{8}^{*}+\theta=t_{5}^{*}<t_{7}^{*} .\right)
$$

Using Corollary 2.2 (ii) and (2.1) we infer

$$
\begin{aligned}
& \phi^{* *}(-1)+\omega-r_{3}+\left[\phi^{* *}(t)-\phi^{* *}(-1)-r_{-4}\right] \leqq h_{b}\left(t_{+}+t\right) \\
& \leqq \phi^{* *}(-1)+\omega+r_{3}+\left[\phi^{* *}(t)-\phi^{* *}(-1)+r_{4}\right],
\end{aligned}
$$

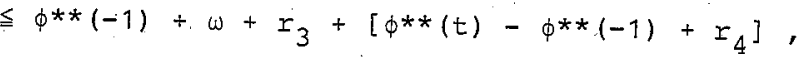

(1)

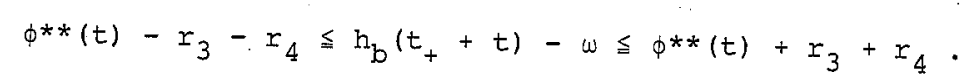

For $b=4$, with (2.2) instead of Corollary 2.2

$$
h_{4}\left(t_{+}+t\right)-\omega \leqq \phi * *(t)-r_{3}+r_{4} .
$$

or $b=b^{*}$, with the last assertion of Corollary 2.2 :

(3) $\phi^{* *}(t)+r_{3}-r_{4} \leqq h_{b *}\left(t_{+}+t\right)-\omega$.

4. Combining (1) with (III.3.3) we obtain

(4) $\phi^{*}(t)-2 r_{3}<h_{b}\left(t_{+}+t\right)-w<\phi^{*}(t)+2 r_{3}$ for $-1 \leqq t \leqq \theta$ and $4 \leqq b \leqq b^{*}$.

Moreover, $\phi^{*}(\theta)=\phi^{* *}(\theta)$, gives.

(5) $\quad \phi^{*}(\theta)-r_{3}-r_{4} \leqq h_{b}\left(t_{+}+\theta\right)-\omega \leqq \phi^{*}(\theta)+r_{3}+r_{4}$

For $b=4,(2)$ and $\phi^{* *} \leqq \phi^{*}$ and $r_{4}<r_{3}$ yield

(6) $h_{4}\left(t_{+}+t\right)-\omega \leqq \phi^{*}(t)-r_{3}+r_{4}<\phi^{*}(t)$ for $-1 \leqq t \leqq \theta$.

For $b=b^{*},(3)$ and (III.3.3) give

(7) $\phi^{*}(t)<h_{b^{*}}\left(t_{+}+t\right)-\omega$ for $-1 \leqq t \leqq \theta$

while

(8) $\phi^{*}(\theta)+r_{3}-r_{4} \leq h_{b *}\left(t_{+}+\theta\right)-\omega$.

5. Estimate of $\dot{h}_{b}\left(t_{+}+\cdot\right)$ for $-\frac{1}{3} \leqq t \leq 0$ and $4 \leqq b \leqq b^{*}$ : $\dot{h}_{b}\left(t_{+}+t\right)=g_{b}\left(\dot{h}_{b}\left(t_{8}^{*}+t\right)\right) \in g_{b} \circ h_{b}\left(\left[t_{z}^{*}, t_{8}^{*}\right]\right)$
$c g_{b}\left(\left[\gamma_{z}, \omega\right]\right)$
$c\left(c_{1}, 0\right)$
(with $\gamma_{z}<h_{b}<\omega$ on $\left(t_{z}^{*}, t_{8}^{*}\right]$
(with (2.3) and Corollary 2.2 (i))

6. Application of Proposition III.3.3 : Let $4 \leq b \leq b^{*}$. With part 5 and (5), we get

$$
\phi^{*}(t)-2 r_{3}<h_{b}\left(t_{+}+t\right)-\omega<\phi^{*}(t)+2 x_{3} \text { for } \theta \leqq t \leqq 0 .
$$

Together with (4) 


$$
h_{b, t_{+}}-\omega \in \phi^{*}+c_{2 x_{3}}=N_{+}
$$

and assertion (ii) is proved.

Tn case $b=4,(6)$ for $t=\theta$ and part 5 permit to use Proposition III.3.3 (iii). It follows that

$$
h_{4}\left(t_{+}+t\right)^{-}-\omega<\phi^{*}(t) \text { for } \theta \leqq t \leqq 0 \text {. }
$$

With (6), we get

$$
\mathrm{h}_{4, \mathrm{t}_{+}}=\omega<\phi^{*} \text {. }
$$

In case $b=b^{*},(8)$ and part 5 permit to use Proposition III.3.3 (ii). It follows that

$$
\phi^{*}(t)<h_{b^{*}}\left(t_{+}+t\right)-\omega \text { for } \theta \leqq t \leq 0 .
$$

With (7), we get

$$
\phi^{*}<h_{b^{*}, t_{+}}-w \text {. }
$$

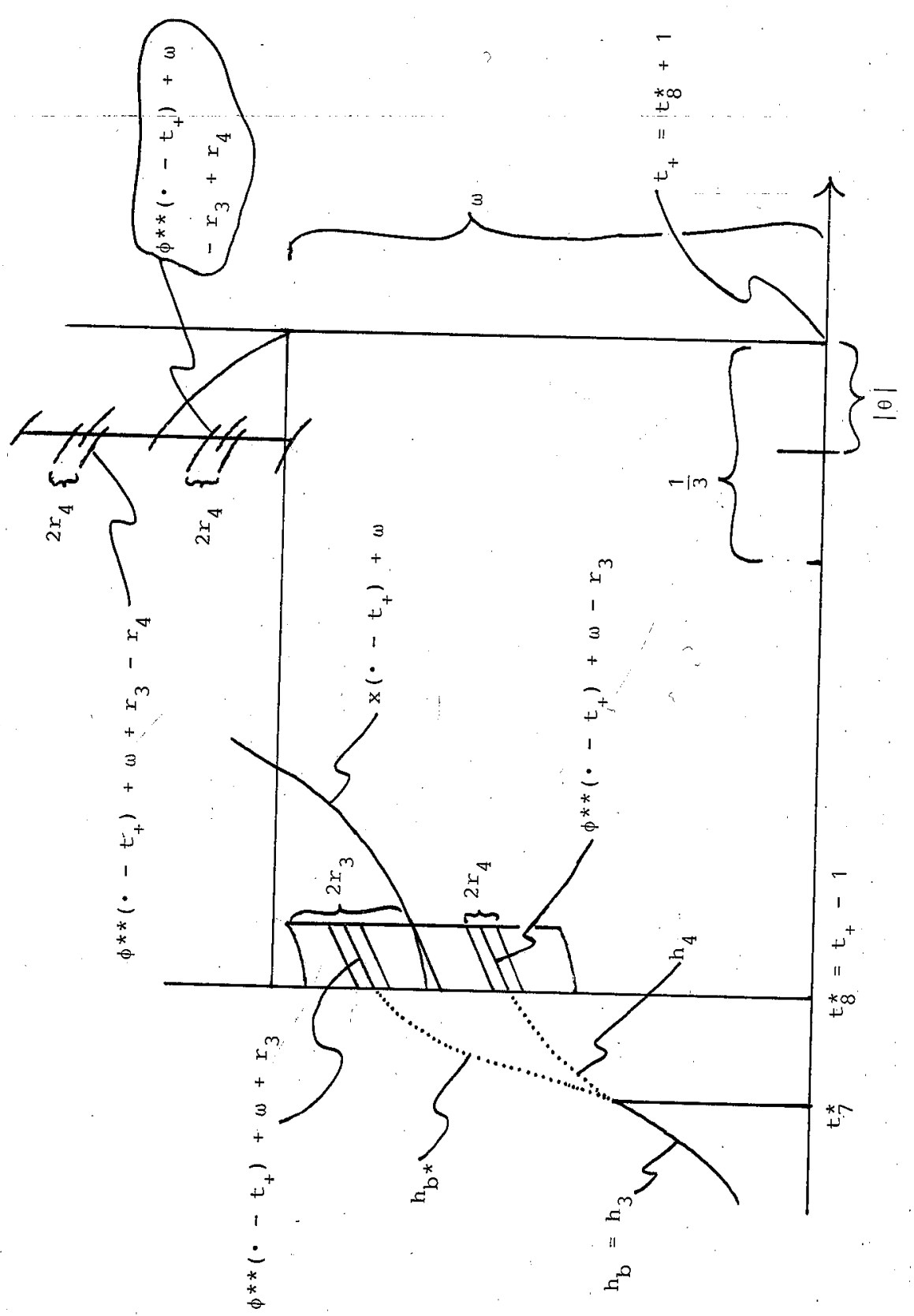


For transversality, we verify a no-zero condition as in proposition III. 4.2 :

PROPOSITION 2. Let $4 \leqq b \leqq b^{*}$. The solution $w_{b}:[-1, \infty) \rightarrow \underline{R}$ of

$$
\dot{w}(t)=g_{b}^{\prime}\left(h_{b}(t-1)\right) w(t-1), w_{0}=v \in T_{X}^{U}
$$

satisfies

$$
w_{b}(t)-c^{*} \dot{h}_{b}(t) \neq 0 \text { for } t_{+}-1 \leqq t \leqq t_{+} \cdot
$$

Before giving the proof, let us describe the concept which led to Proposition 2 .

RFMARK 1. The foundations for Proposition 2 were laid in section 1 , and by (2.4). Proposition 1.2 (ii) can now be restated as

(*) $\quad w_{b}<c^{*} \dot{h}_{b}$ on $\left[t_{2}^{*}, t_{3}^{*}\right)$, or $w_{b}>c^{*} \dot{h}_{b}$ on $\left[t_{2}^{*}, t_{3}^{*}\right)$.

An inequality like

$$
w_{b}<c^{*} \dot{h}_{b} \text { on }\left[t_{+}-1, t_{+}\right]=\left[t_{8}^{*}, t_{8}^{*}+1\right]
$$

relies on behavior of $w_{b}$ and $\dot{h}_{b}$ on the interval $\left[t_{2}^{*}, t_{2}^{*}+1\right]=$

$\left[t_{2}^{*}, t_{6}^{*}\right]$ and on sign conditions for the coefficient $g_{b}^{\prime}\left(h_{b}(\cdot-1)\right)$ in the

linear differential equation for $w_{b}$ and $c^{*} \dot{h}_{b}$.

$g_{b}$ was designed to steer $h_{b, t}$ close to $\phi^{*}$. This forces the sign of $g_{b}^{\prime}\left(h_{b}(\cdot-1)\right)$ to change several times on the interval $\left[t_{2}^{*}, t_{8}^{*}+1\right]$, aid seems difficult if not hopeless to control the sign of the solution $w_{b}-c^{*} \dot{h}_{b}$ on the interval $\left[t_{6}^{*}, t_{8}^{*}+1\right]$.

The construction of $g_{b}$ with $g_{2}^{\prime}(\xi)=0$ for all $\xi \geqq h_{1}\left(\left[t_{1}^{*}+1\right]\right)$ implies that $w_{b}$ and $\dot{h}_{b}$ become constant on $\left[t_{3}^{*}, t_{6}^{*}\right]$. By the choice of $c^{*}, w_{b}=c^{*} h_{b}$ on this interval - so that the linear variational equation shows that $w_{b}$ and $c^{*} \dot{h}_{b}$ differ by a constant on the interval

$$
\left[t_{3}^{*}+1, t_{6}^{*}+1\right]=\left[t_{7}^{*}, t_{6}^{*}+1\right] \text {, }
$$

no matter how oscillatory the coefficient $g_{b}^{\prime}\left(h_{b}(\cdot-1)\right)$ is on the interval $\left[t^{\frac{k}{7}}, t_{6}^{*}+1\right]:$

In order to determine the sign of this constant, i.e.

$$
\operatorname{sign}\left[w_{b}\left(t_{7}^{*}\right)-c^{*} \dot{h}_{b}\left(t_{7}^{*}\right)\right] \text {, }
$$

we have

$$
w_{b}\left(t_{6}^{*}\right)-c^{*} \dot{h}_{b}\left(t_{6}^{*}\right)=0
$$

作 a result provided there is a sign condition for the coefficient $g_{b}^{\prime}\left(h_{b}(\cdot-1)\right)$ on the interval $\left(t_{6}^{*}, t_{7}^{*}\right]$.

such a sign condition is

$$
0<g_{3}^{\prime} \text { on }\left(\gamma_{2}, \infty\right)=\left(\gamma_{2}, \gamma_{3}\right]
$$

from Section 1 .

For the final comparison of $w_{b}$ and $c^{*} \dot{h}_{b}$ on the remaining "small" interval $\left[t_{6}^{*}+1, t_{8}^{*}+1\right]$ we need

$$
0<g_{4}^{\prime} \text { on }\left[\gamma_{6}, \gamma_{8}\right) \text {, }
$$

i.e., the condition (2.4).

PROOF of Proposition 2. 1. We show $h_{b}=h_{2}$ on $\left(-\infty, t_{6}^{*}\right]$ and $w_{b}=w_{2}$ on $\left[-1, t_{6}^{*}\right]$ : For $t \leqq t_{6}^{*}=t_{2}^{*}+1$,

$$
h_{2}(t)=h_{3}(t)=h_{b}(t) \quad(\text { see }(1.3) \text { and Proposition } 1) .
$$

Let $0 \leqq t \leqq t_{6}^{*}$. Then $-1 \leqq t-1 \leqq t_{2}^{*}$, and therefore

$$
-\gamma<h_{3}(t-1) \leqq h_{3}\left(t \frac{*}{3}\right)=\gamma_{3} \quad \text { (see (1.3): }
$$

With $g_{b}=g_{3}$ on $\left[-\gamma, \gamma_{3}\right]$,

$$
g_{b}^{\prime}\left(h_{b}(t-1)\right)=g_{3}^{\prime}\left(h_{2}(t-1)\right)=g_{2}^{\prime}\left(h_{2}(t-1)\right)
$$

(recall

$$
-\gamma<h_{2}(t-1) \leq h_{2}\left(t_{2}^{*}\right)=\gamma_{2}
$$

and $g_{3}=g_{2}$ on $\left[-\gamma, \gamma_{2}\right]$ ). It follows that $w_{b}$ and $w_{2}$ satisfy the same linear differential delay equation for $0 \leqq t \leq t_{6}^{*}$, and

$$
\mathrm{w}_{\mathrm{b}, 0}=\mathrm{v}=\mathrm{w}_{2,0} \text {. }
$$

2. Part 1, the choice of $\mathrm{C}^{*}$ in Proposition 1.2 and

$$
\left[t_{1}^{*}+1\right]+1=t_{3}^{*}<t_{6}^{*}
$$

yield

(9) $w_{b}\left(t_{3}^{*}\right)=c^{*} \dot{h}_{b}\left(t \frac{*}{3}\right)$.

Part 1, Proposition 1.2 (ii) and

$$
t_{3}^{*}-\varepsilon^{*} \leqq t_{2}^{*}<t_{3}^{*}
$$

give

(10) $w_{b}(t) \neq c^{*} \dot{h}_{b}(t)$ for $t_{2}^{*} \leqq t<t_{3}^{*}$.

From part 1 and from

$$
\dot{w}_{2}(t)=0=\ddot{h}_{2}(t) \text { for } t \geqq\left[t_{1}^{*}+1\right]+1=t_{3}^{*}
$$

we obtain

$$
\dot{\mathrm{w}}_{\mathrm{b}}=0=\mathrm{c}^{*} \ddot{\mathrm{h}}_{\mathrm{b}} \text { on }\left[t_{3}^{*}, t_{6}^{*}\right]
$$




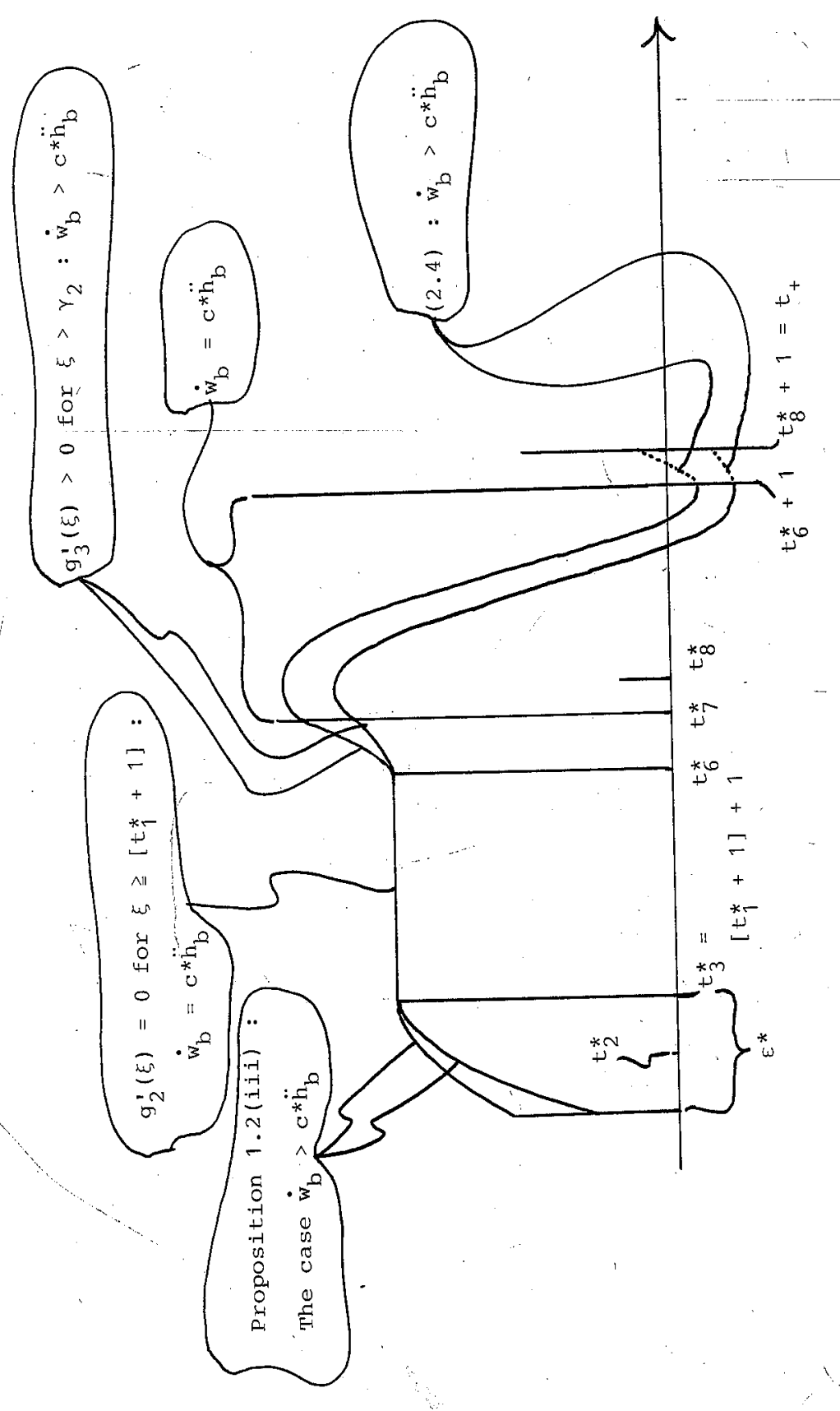

so that (9) implies

(11) $w_{b}=c^{*} \dot{h}_{b}$ on $\left[t_{3}^{*}, t_{6}^{*}\right]$.
3. Suppose
(12) $w_{b}>c^{*} \dot{h}_{b}$ on $\left[t_{2}^{*}, t_{3}^{*}\right)$.

We show $w_{b}>c^{*} \dot{h}_{b}$ on $\left(t_{6}^{*}, t_{6}^{*}+1\right]$ :

3.1. Let $t_{6}^{*}<t<t_{7}^{*}$. Then $t_{2}^{*}<t-1<t_{3}^{*}$, and $g_{b}^{\prime}\left(h_{b}(t-1)\right)=g_{3}^{\prime}\left(h_{3}(t-1)\right)>0$

(with

$$
r_{2}=h_{3}\left(t_{2}^{*}\right)<h_{3}(t-1)
$$

and $0<g_{3}^{\prime}$ on $\left(\gamma_{2}, \infty\right)$ )

so that (12) implies

$\dot{w}_{b}(t)=g_{b}^{\prime}\left(h_{b}(t-1)\right) w_{b}(t-1)>g_{b}^{\prime}\left(h_{b}(t-1)\right) c^{*} \dot{h}_{b}(t-1)=c^{*} \ddot{h}_{b}(t)$.

3.2. For $t_{7}^{*} \leqq t \leqq t_{6}^{*}+1, t_{3}^{*} \leqq t-1 \leqq t_{6}^{*}$, and (11) gives

$\dot{w}_{b}(t)=g_{b}^{\prime}\left(h_{b}(t-1)\right) w_{b}(t-1)=g_{b}^{\prime}\left(h_{b}(t-1)\right) c^{*} \dot{h}_{b}(t-1)=c^{*} \ddot{h}_{b}(t)$.

3.3. Using (11) for $t=t_{6}^{*}$ and the results of parts 3.1 and 3.2 we obtain the assertion.

4. Proof of $g_{b}^{\prime}\left(h_{b}(t-1)\right)>0$ for $t_{6}^{*}+1 \leqq t \leqq t_{8}^{*}+1$ :

Let $t_{6}^{*} \leqq t-1 \leqq t_{8}^{*}$. From Proposition 1,

$h_{b}\left(t_{6}^{*}\right)=h_{3}\left(t_{6}^{*}\right)=\gamma_{6}, 0^{\prime}<\dot{h}_{b}$ in $\left(t_{z}, t_{8}^{*}\right]>\left[t_{6}^{*}, t_{8}^{*}\right]$,

$h_{b}\left(t_{8}^{*}\right)<\omega$.

It follows that $h_{b}(t-1) \in\left[\gamma_{6}, \omega\right]$. Recall

$$
\begin{array}{lll}
g_{b}^{\prime}=g_{4}^{\prime} \text { on }\left[\gamma_{4}, \omega+\gamma\right]=\left[\gamma_{6}, \omega\right] & \text { (Corollary } 2.2 \text { (i)), } \\
g_{4}^{\prime}>0 \text { on }\left[\gamma_{6}, \gamma_{8}\right] & \text { (see }(2.4)), \\
g_{4}^{\prime}=g_{1}^{\prime}(\cdot-\omega)>0 \text { on }\left[\gamma_{8}, \omega+\gamma\right] \supset\left[\gamma_{8}, \omega\right] .
\end{array}
$$

5. In case $(12), w_{b}>c^{*} \dot{h}_{b}$ on $\left[t_{+}-1, t_{+}\right]=\left[t_{8}^{*}, t_{8}^{*}+1\right]$. Proof: By part 3,

$$
w_{b}\left(t_{6}^{*}+1\right)>c^{*} \dot{h}_{b}\left(t_{6}^{*}+1\right) \text {, }
$$

and for $t_{6}^{*}+1<t<t_{8}^{*}+1$,

$$
\dot{w}_{b}(t)=g_{b}^{\prime}\left(h_{b}(t-1)\right) w_{b}(t-1)>g_{b}^{\prime}\left(h_{b}(t-1)\right) c^{*} \dot{h}_{b}(t-1)=c^{*} \ddot{h}_{b}(t)
$$

(with parts 4 and $3 ; t_{6}^{*}<t-1<t_{\frac{*}{8}}<t_{\frac{*}{6}}+1$ ), so that

$$
w_{b}>c^{*} \dot{h}_{b} \text { on }\left[t_{6}^{*}+1, t_{8}^{*}+1\right] \text {. }
$$

Recall part 3 , and $t_{6}^{*}<t_{8}^{*}<t_{6}^{*}+1$.

6. The proof for the case $\mathrm{w}_{\mathrm{b}}<\mathrm{c}^{*} \dot{h}_{\mathrm{b}}$ on $\left[t_{2}^{*}, t_{3}^{*}\right)$ is analogous. 
4. HETEROCLINIC SOLUTIONS AND TRANSVERSAL HOMOCLINIC POINTS

The construction of the functions $g_{b}$ yields

COROLIARY 1. (Heteroclinic solutions) There exists $b \in\left(4, b^{*}\right)$ with

$h_{b, t} \rightarrow 0+w$ as $t+\infty, h_{b, t} \rightarrow 0$ as $t \rightarrow-\infty$.

we have

$$
\begin{aligned}
& \underline{P}\left(h_{b}, t_{+}-w\right) \in S \subset H, \\
& h_{b}, t_{n}=x_{n} \text { for } n \in-\underline{N}_{0} .
\end{aligned}
$$$$
\left|h_{b}(t)-\omega\right|<\frac{\gamma}{2} \text { for } t \geq t_{+}-1 \text { ! }
$$$$
\left|h_{b}(t)\right|<\frac{\gamma}{2} \text { for } t \leqq 0 \text {. }
$$

PROOF. 1. The map $\left[4, b^{*}\right] \ni b \rightarrow h_{b, t_{+}} \in C$ is continuous, with

$$
\begin{aligned}
& x_{b}^{+}:=h_{b}, t_{+}-\omega \in N_{+} \text {for } 4 \leqq b \leqq b^{*}, \\
& x_{4}^{+}<\phi^{*}<x_{b^{*}}^{+}
\end{aligned}
$$

Proposition III.3.1 implies that each $\underline{P}\left(X_{b}^{+}\right) \in \cdot H$ is below, on or above $S$, with $\underline{P}\left(x_{4}^{+}\right)$below $S$ and $\underline{P}\left(x_{b^{*}}^{+}\right)$above $S$. By continuity, we obtain $b \in\left(4, b^{*}\right)$ with $\underline{P}\left(x_{b}^{+}\right) \in S$.

2. The solution $y:[-1, \infty) \rightarrow \underline{R}$ of eq. $\left(g_{1}\right)$ with $y_{0}=x_{b}^{+}=h_{b}, t_{+}-w$ satisfies

$$
|y(t)|<\frac{\gamma}{2} \text { for } t \in[-1,2]>\left[-1, \tau\left(x_{b}^{+}\right)\right]
$$

and $y_{\tau_{+}}=\underline{P}\left(X_{b}^{+}\right) \in S$ where $\tau_{+}:=\tau\left(X_{b}^{+}\right) \cdot$ With Corollaries II.1.1 $(i)$ and III.2.1, we obtain

$$
\begin{aligned}
& |y(t)|<\frac{\gamma}{2} \text { for all } t \geq-1, \\
& p^{n}\left(y_{\tau_{+}}\right) \rightarrow \phi^{*} \text { as } n \rightarrow \infty .
\end{aligned}
$$

$T(N) \subset(1,2)$ and continuous dependence on initial data yield $y_{t} \rightarrow \circ$ as $t \rightarrow \infty \cdot g_{b}=g_{b}(\cdot+\omega)$ implies that $h_{b}\left(\cdot+t_{+}\right)-\omega$ is a solution of eq. $\left(g_{b}\right)$. With $g_{b}=g_{1}$ on $[-\gamma, \gamma]$ and $|y|<\frac{\gamma}{2}$, we infer $h_{b}\left(t+t_{+}\right)-w=y(t)$ for $t \geqq-1$.
3. The assertions for $t \leq 0$ and $n \in-N$ follow from Proposition 1.1 since $h_{b}=h_{3}=h_{1}$ on $(-\infty, 0]$.

From now on we consider $g:=g_{b}$ and $h:=h_{b}$ given by corollary 1 . The index $b$ is dropped whenever convenient:

The next result exhibits a difference to the examples of chaotic differential delay equations from $[32,23,10,11,9]$ :

PROPOSITION 1. $h_{t} \notin 0+w$ for all $t \geq t_{+}$.

COMMENT. In $[32,10,11,9]$, nonlinearities were carefully designed smoothed step functions so that solutions of the differential delay equations could explicitly be computed. The semiflow entered a 2-dimensional subset of $c$ containing a periodic orbit, and there was a homoclinic trajectory which merged into the periodic orbit in finite time.

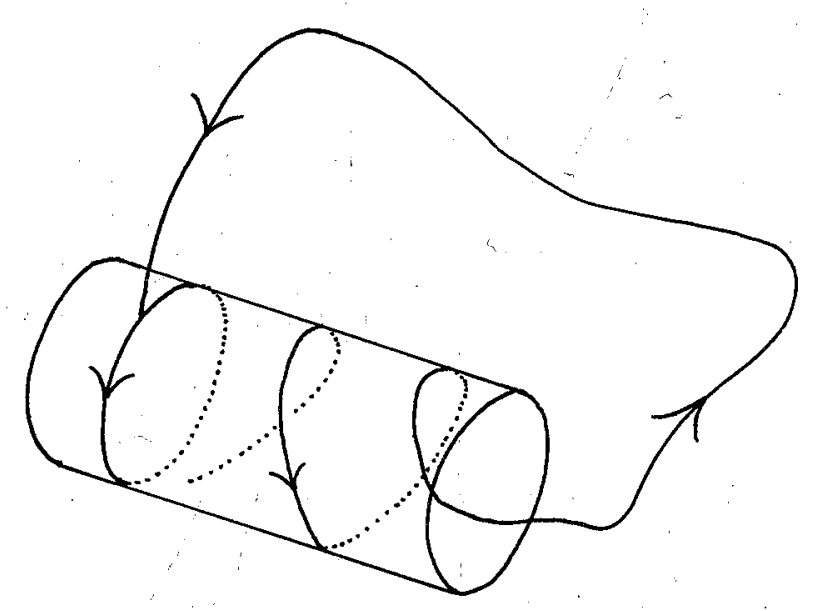

Both facts together made it possible to obtain a poincare'map on a one-dimensional subset, with a "snap-back repeller" [16] (see Preliminaries) - so that chaotic motion in $[32,10,11]$ was a consequence of results for interval maps $[16,15,24]$.

Proposition 1 shows that the present situation is not as simple. 
PROOF of Proposition 1. 1. Proof of $h_{t_{+}}-\omega \neq x_{t}$ for all $t \in \underline{R}$ : $h(t)=h\left(t_{+}-1\right) \geqq x(-1)+\omega-2 x_{3} \quad$ (Proposition 3.1 (ii)) $>-\frac{\gamma}{4}+\omega-\frac{\gamma}{2} \quad$ (choice of. $r_{3}$ in section III.3) $>\omega-\gamma=\gamma_{8}$

and

$$
\begin{aligned}
& h\left(t_{8}^{*}+\theta\right)=h\left(t_{\frac{*}{8}}^{*}\right) \leqq h\left(t_{7}^{*}\right) \\
& =h_{3}\left(t_{7}^{*}\right) \\
& =\gamma_{7}<\gamma_{8}
\end{aligned}
$$

imply that for some $t \in[\theta, 0], h(t * t)=\gamma_{8}$. Therefore

$$
\dot{h}\left(t_{+}+t\right)=g\left(h\left(t_{+}+t-1\right)\right)=g\left(\gamma_{8}\right)=g(-\gamma)=g_{1}(-\gamma),
$$

while

$\dot{x}\left(t^{\prime}\right) \in g_{1}\left(\left[-\frac{\gamma}{4}, \frac{\gamma}{4}\right]\right) \quad$ for alli $t^{\prime} \in \underline{\underline{R}}$.

2. Let $t \in \underline{R}$. The solutions $h\left(\cdot+t_{+}\right)-w$ and $x(\cdot+t)$ of eq.

$(g)$ are bounded on $[-1, \infty)$ by $\frac{\gamma}{2}$ (Corollary 1$)$. The restriction of $g$ to the interval $[-\gamma, \gamma]$ is injective since $0<g_{1}^{\prime}=g^{\prime}$. on $[-\gamma, \gamma]$. Using part 1 and eq. $(g)$ one concludes by induction that

$$
h_{t_{+}}+n-\omega \neq x_{t+n} \text { for all } n \in \mathbb{N}_{0} \text { and all } t \in \underline{R} .
$$

Suppose now that

$$
h_{t}-\omega=x_{\underline{t}} \text { for some } t \geq t_{+} \text {and some } \underline{t} \in \underline{R} \text {. }
$$

It follows that the solutions $h-\omega$ and $x(\cdot+\underline{t}-t)$ of eq. (g) coincide on the interval $[t-1, \infty)$. Hence

$$
h_{t_{+}+n}-w=x_{t_{+}}+n+\underline{t}-t \text { for some } n \in \underline{\mathbb{N}}_{0},
$$

a contradiction.

We complete the points $x_{n}, n \in-\underline{\mathbb{N}}_{0}$, to a sequence in $D \underline{\underline{Z}}$ which tends to $\phi^{*}$ as $n \rightarrow \infty$ : set $\chi^{+}:=h_{t_{+}}-\omega$ (as in the proof of corollary , 1), and $x_{1}:=\underline{P}\left(x^{+}\right) \in S, t_{1}:=t_{+}^{+} \tau\left(x^{+}\right)$. It follows that there are a sequence of points $x_{n}=P\left(x_{n-1}\right) \in S, 2 \leq n \in \underline{\mathbb{N}}$, with

$$
x_{n} \rightarrow \phi^{*} \text { as } n+\infty, \cdot
$$

and a sequence of reals $t_{n}, 2 \leqq n \in \underline{N}$, with

$$
\begin{aligned}
& t_{n}=t_{n-1}+\tau\left(x_{n-1}\right) \in t_{n-1}+(1,2) \text { for all integers } n \geqq 2 ; \\
& t_{n}+\infty \text { as } n \rightarrow \infty .
\end{aligned}
$$

COROLIARY 2. (i) $x_{n}=h_{t_{n}}$ - $\omega$ for all $n \in \underline{\mathbb{N}}$.

(ii) $x_{n} \neq \phi *$ for all $n \in \underline{z}$.

(iii) There, exists $r_{5}>0$ with the following properties.

(iii.1) $\phi^{*} \notin x_{0}+C l{ }_{r_{5}} \subset D$.

(iii.2) $x_{n} \notin x_{0}+c l H_{x_{5}}$ for all $n \in \underline{z} \backslash\{0\}$.

(iii.3) For every solution $y:[-1, \infty)+\underline{R}$ of eq. (g) with $y_{0} \in x_{0}+H_{r_{5}}$

$$
\mathrm{y}_{\mathrm{t}_{+}}-\omega \in \mathrm{N}_{+} \text {. }
$$

PROOF. 1. $\omega$-periodicity of $g$ implies that $h-\omega$ is a solution of eq. (g). $g=g_{1}$ on $[-\gamma, \gamma]$ and Corollary 1 show that the restriction of $\mathrm{h}-\omega$ to the interval $\left[t_{+}-1, \infty\right)$ is a solution of eq. $\left(g_{1}\right)$. Now assertion (i) is obvious from the definitions of $x^{+},\left(x_{n}\right)_{1}^{\infty}, \underline{p}, P$.

2. Proof of assertion (ii) : Assertion (i), Proposition 1 and $t_{n}>t_{+}$ exclude $x_{n}=\phi^{*}$ for $n \in \underline{N}$. Suppose $x_{n}=\phi^{*}$ for some $n \in-\underline{N}_{0}$. Then $h_{t_{n}}=\phi^{*}$ (with Corollary 1), and consequently

$$
\begin{aligned}
& h_{t}=x_{t}-t_{n} \in \circ \text { for } t \geqq t_{n}, \\
& |h(t)|<\frac{y}{4}<\omega \text { for, } t \geqq t_{n}-1,
\end{aligned}
$$

a contrajiction to

$$
t_{n}-1<0<t_{+} \text {and }\left|h\left(t_{+}\right)\right|+\frac{\gamma}{2}>\omega
$$

(Corollary 1).

3. Suppose $x_{n}=x_{0}$ with $0 \neq n \in \underline{z}$. In case $n>0, h_{0}=h_{t_{n}}-\omega$ so that the solutions $h$ and $h\left(\cdot+t_{n}\right)-w$ of eq. $(g)$ coincide on

$[-1, \infty)$. With $0<t_{n}$,

$$
h\left(j t_{n}\right)=h(0)+j \omega \rightarrow \infty \text { as } \underline{N} \exists j \rightarrow \infty \text {, }
$$

a. contradiction to Corollary' 1 .

In case $n<0, h_{t_{n}}=h_{0}$, and $h\left(\cdot+t_{n}\right)=h$ on $[-1, \infty)$. For $j$

in $\underline{N}$ with $t_{+}+j t_{n} \in\left(t_{n}, 0\right]=\underline{R}^{-}$,

$$
h\left(t_{+}\right)=h\left(t_{+}+j t_{n}\right) \in h\left(\underline{R}^{-}\right) \in\left(-\frac{\gamma}{2}, \frac{\gamma}{2}\right),
$$

a contradiction to Corollary 1 .

4. In view of assertion (ii), part 3 and $x_{n} \rightarrow \phi^{*}$ as $|n| \rightarrow \infty$, there exists $r>0$ with

$$
\phi^{*} \not x_{0}+\mathrm{cl} \mathrm{H}_{x} \not \partial \chi_{\mathrm{n}} \text { for } 0 \neq \mathrm{n} \in \underline{z} \text {. }
$$

$h_{0}=x_{0}$ and $h_{t_{+}}-\omega \in N_{+}$permit to find $r_{5} \in(0, r)$ so small that 
$x_{0}+c l H_{r_{5}} \subset D$, and for all solutions $y:[-1, \infty) \rightarrow \underline{R}$ of eq. $(g)$ with $y_{0} \in x_{0}+E_{r_{5}}, \dot{y}_{t_{+}}-\omega \in N_{+}$.

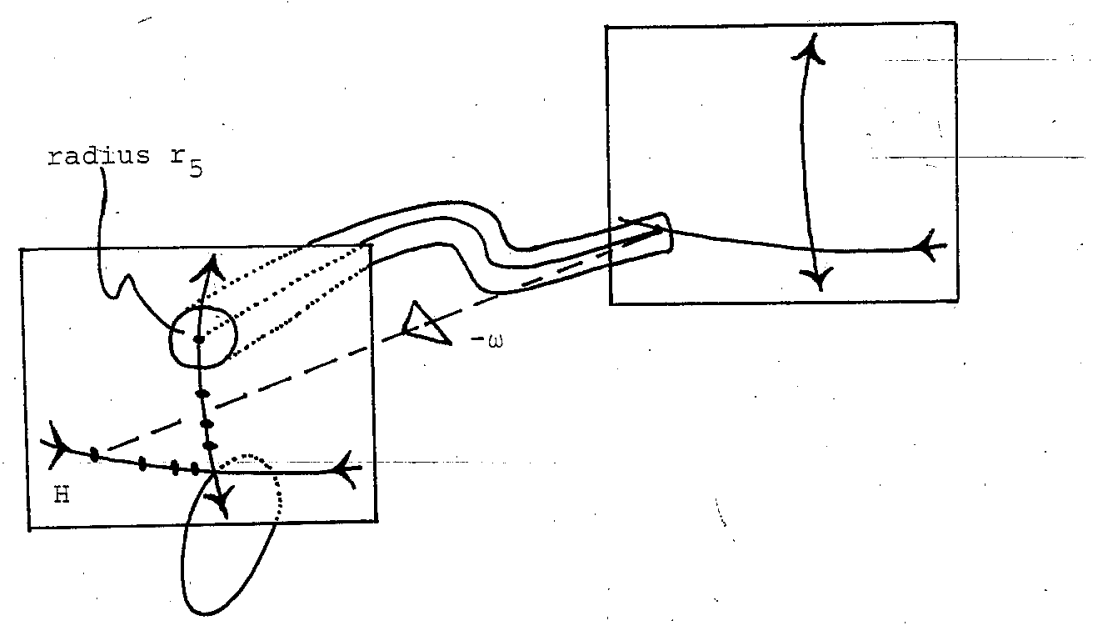

We modify the Poincare'map $P$ so that $\left(x_{n}\right)_{-\infty}^{\infty}$ becomes a homoclinic trajectory. With $r_{5}$, according to Corollary 2, we set

$$
\begin{aligned}
& D^{\prime}:=\left\{\phi \in|D:| \phi-x_{0} \mid \neq r_{5}\right\}, \\
& P^{\prime}(\phi):=P(\phi) \text { if } \phi \in D \text { and }\left|\phi-x_{0}\right|>r_{5}, \\
& P^{\prime}(\phi):=P\left(y_{t_{+}}-\omega\right) \text { for } \phi \in x_{0}+H_{r_{5}} \text { where } y:[-1, \infty) \rightarrow \underline{R} \text { is } \\
& \text { the solution of eq. (g) with } \mathrm{y}_{0}=\phi .
\end{aligned}
$$

The map $P^{\prime}$ is $C^{1}$. On the open subset $D-\left(X_{0}+c l H_{r_{5}}\right)$ of $D, P^{\prime}$ is a shift along solutions $y$ until a return of their segments $y_{t}$ to $H$. on the open subset $X_{0}+\mathrm{H}_{r_{5}}$ of $H, P^{\prime}$ describes solutions whose segments pass through $\mathrm{N}_{+}+\omega$ and hit the hyperplane $\mathrm{H}+\omega$ afterwards.

COROLIARY 3. $\phi^{*}$ is a hyperbolic fixed point of $P^{\prime}$, and $\left(x_{n}\right)_{-\infty}^{\infty}$ is a homoclinic trajectory of $P^{\prime}$.

PROOF. We show $P^{\prime}\left(x_{n}\right)=x_{n+1}$ for $n \in \underline{z}$ : By definition of $x_{1}$ and $P^{\prime}$,

$$
X_{1}=\underline{P}\left(h_{t_{+}}-\omega\right)=P^{\prime}\left(h_{0}\right)=P^{\prime}\left(x_{0}\right) .
$$

For $\mathrm{n} \neq 0, x_{\mathrm{n}} \in \mathrm{D}$ and $\left|x_{\mathrm{n}}-x_{0}\right|>r_{5}$ (Corollary 2) so that $\mathrm{P}^{\prime}\left(x_{\mathrm{n}}\right)=$ $P\left(x_{n}\right)$. If $n<0, P\left(x_{n}\right)=x_{n+1}$ by Proposition 1.1. If $n>0$, $P\left(x_{n}\right)=x_{n+1}$ by the construction before corollary 2 .

Transversality: Consider the tangent vector

$$
\mathrm{v}_{0}:=\mathrm{v} \in \mathrm{T}_{\chi_{0}}^{\mathrm{U}}
$$

and its forward iterates defined by

$$
v_{n+1}=D P^{\prime}\left(x_{n}\right) v_{n} \text { for } n \in \underline{N}_{0} \text {. }
$$

Recall $x_{n} \in S$ for $n \in \underline{N}$.

COROLLARY 4. $\mathrm{v}_{\mathrm{n}} \notin \mathrm{T}_{\mathrm{X}_{\mathrm{n}}} \mathrm{S}$ for all $\mathrm{n} \in \underline{\mathrm{N}}$.

PROOF. 1. Let $X$ denote the semiflow given by the solutions $y:[-1, \infty) \rightarrow \underline{R}$ of eq. $(g)$. We have

$$
\begin{aligned}
\mathrm{v}_{1} & =\mathrm{DP}\left(\mathrm{x}_{0}\right) \mathrm{v}_{0}=\left[\mathrm{DP}\left(\mathrm{X}\left(t_{+}, x_{0}\right)-\omega\right) \circ \mathrm{D}_{2} \mathrm{x}\left(t_{+}, x_{0}\right)\right] \mathrm{v}_{0} \\
& =D \underline{P}\left(h_{t_{+}}-\omega\right) \mathrm{w}_{t_{+}}
\end{aligned}
$$

with the solution $w:[-1, \infty) \rightarrow \underline{R}$ of

$$
\dot{w}(t)=g^{\prime}(h(t-1)) w(t-1), w_{0}=v_{0} \text {. }
$$

2. Application of Proposition III.4.2 to $\mathrm{y}^{\prime}:=h\left(\cdot+t_{+}\right)-\omega$ and $\underline{w}:=w\left(\cdot+t_{+}\right)$: Corollary' 1 and $g=g_{1}$ on the interval $[-\gamma, \gamma]$ imply that for all $t>0$,

$$
\dot{y}(t)=\dot{h}\left(t+t_{+}\right)=g\left(h\left(t+t_{+}-1\right)\right)=g\left(h\left(t+t_{+}-1\right)-\omega\right)
$$

$$
=g_{1}(y(t-1)) \text {. }
$$

Note $\mathrm{y}_{0} \in \mathbb{N}_{+}$and $\underline{P}\left(\mathrm{y}_{0}\right)=x_{1} \in \mathrm{S}$. For all $t>0$,

$$
\begin{aligned}
\underline{\dot{w}}(t) & =\dot{w}\left(t+t_{+}\right)=g^{\prime}\left(h\left(t+t_{+}-1\right)\right) w\left(t+t_{+}-1\right) \\
& =g_{1}^{\prime}(y(t .1)) \underline{w}(t-1) .
\end{aligned}
$$

Proposition 3.2 guarantees that $\underline{w}_{0}-c^{*} \dot{y}_{0}=w_{t_{+}}-c^{*} \dot{h}_{t_{+}}$has no zero. It follows that for some $t \geqq 0, \underline{w}_{t}=w_{t}+t_{+}$has no zero.

3. Application of Proposition III.4.1 : For all $n \in \underline{N}$, $x_{n+1}=P^{\prime}\left(x_{n}\right)=P\left(x_{n}\right)$ and $v_{n+1}=D^{\prime}\left(x_{n}\right) v_{n}=D P\left(x_{n}\right) v_{n}$ so that we obtain

$$
v_{1}=D \underline{P}\left(h_{t_{+}}-\omega\right) w_{t_{+}}=D \underline{P}\left(y_{0}\right) w_{0} \notin T_{\underline{P}\left(y_{0}\right)} S=T_{x_{1}} S,
$$

and for $2 \leqq n \in \underline{N}$,

$$
v_{n} \notin T_{X_{n}} s
$$


V. ON CHAOTIC BEHAVIOR

A prototype of chaotic behavior is given by the shift in two symbols

$$
\sigma:\left(b_{n}\right)_{n} \in \underline{z} \rightarrow\left(b_{n+1}\right)_{n} \in \underline{z}
$$

on the space $\{0,1\} \underline{Z}$. With the discrete topology on the symbol space $\{0,1\}$ and the product topology on the sequence space, the latter is compact, and the shift is a homeomorphism with complicated trajectories. See [6] for details.

Smale $[26,27]$ proved that diffeomorphisms with transversal homoclinic points have invariant sets on which iterates are topologically conjugate to a symbol shift. See also $[17,19,21,22]$ and Silnikov's work [25].

These results can be generalized to smooth maps which are not necessarily injective. See [8], or the approach in [29] where hyperbolic structures for arbitrary $c^{1}$-maps and shadowing are used.

of course, one can not expect that restrictions of such maps are equivalent to a shift, which is one-to-one. Nontrivial equivariance seems possible, or better, equivalence of a symbol shift with the shift induced by the map considered on a space of trajectories.

The latter is what Theorem 5.2 [8] asserts. To describe this more precisely, let $F: V \rightarrow I$ be a map on an open subset $V$ of a real Banach space $L$. (This is a bit more general than in [8] where $V=I$. .) The trajectories $\left(y_{n}\right)_{-\infty}^{\infty}$ of $F$ form a closed subset $Y$ of $V \underline{Z}$ (with the discrete topology on $V$ ). On $Y, F$ acts as the shift

$$
\sigma_{F}:\left(y_{n}\right)_{n \in \underline{z}} \rightarrow\left(y_{n+1}\right)_{n \in \underline{z}}=\left(F\left(y_{n}\right)\right)_{n \in \underline{z}} \cdot
$$

For a finite collection of pairwise disjoint open subsets $\Sigma_{0}, \ldots, \Sigma_{\mathrm{m}}$ of $V$, let $I_{\Sigma}$ denote the map from the set $Y_{\Sigma}$ of trajectories in $\Sigma_{0} \cup \ldots \cup \Sigma_{m}$ into the space $\Sigma_{0}:=\left\{\Sigma_{0}, \ldots, \Sigma_{m}\right\}^{z}$ defined by $I_{\Sigma}(y)_{n}=\Sigma_{\mu}$ if $y_{n} \in \Sigma_{\mu}$.

With the discrete topology on the symbol space $\left\{\Sigma_{0}, \ldots, \Sigma_{m}\right\}, I_{\Sigma}$ becomes continuous.

Suppose $F$ is $C^{1}, 0 \in \mathrm{V}$ is a hyperbolic fixed point, and there is a homoclinic trajectory $\left(\mathrm{y}_{\mathrm{n}}\right)_{-\infty}^{\infty}$. The result of Hale and Lin guarantees (under additional hypotheses including transversality) that there exist an integer $k>0$ and sets $\Sigma_{0}, \ldots, \Sigma_{m} ; m \geqq 2$, as above with

$0 \in \Sigma_{0}$ and $y_{n} \in \Sigma_{0} \cup \ldots \cup \Sigma_{m}$ for all $n \in \underline{z}$

such that $I_{\Sigma}$ defines a homeomorphism onto the subset $\Sigma_{I} \in \Sigma$ given by $b_{i+1}=\Sigma_{j+1}$ for $j=1, \ldots, m$ in case $b_{i}=\Sigma_{j}$,

$b_{i+j}=\Sigma_{0}$ for $j=1, \ldots, k$ in case $b_{i}=\Sigma_{m}$ $b_{i+1}=\Sigma_{1}$

in case $b_{i}=\Sigma_{0} \neq b_{i+1}$

which makes the diagram

$\begin{array}{cll} & \stackrel{\sigma_{F}}{ } \\ \mathrm{Y}_{\Sigma} & \rightarrow & \mathrm{Y}_{\Sigma} \\ I_{\Sigma} \downarrow & & \psi \mathrm{I}_{\Sigma} \\ \Sigma_{I} & \vec{\sigma} & \Sigma_{I}\end{array}$

commutative.

The additional hypotheses mentioned above require an equivalent norm

||$*$ on $I$, constants $q \in(0,1), r=r_{F}>0, c=c_{F}>0$ with $q+c<1$

and an integer $j=j_{F}>0$ so that the following holds, with $A=D F(0)$.

(1) $|A v|^{*} \geqq q^{-1}|v| *$ on the linear unstable space $I^{u}$ $|A v| *^{\prime} \leq q|v| *$ on the linear stable space $L^{S}$.

(2) $\left|\mathrm{p}^{\mathrm{u}}[D F(\mathrm{Y})-\mathrm{A}]\right| *<\mathrm{C}$ and $\left|\mathrm{p}^{\mathrm{s}}[D F(\mathrm{y})-\mathrm{A}]\right|^{*}<\mathrm{c}$ for all $\mathrm{y}$ in $\mathrm{cl} \mathrm{L}_{x}^{*}=\left\{y \in L:|y|^{*} \leqq x\right\}$, with the projections $\mathrm{p}^{\mathrm{u}}$ of $\mathrm{I}$ onto $L^{\mathrm{U}}$ and $p^{\mathrm{S}}$ of $L$ onto $L^{\mathrm{S}}$ given by $L=L^{\mathrm{U}} \oplus \mathrm{L}^{\mathrm{S}}$.

(3) $\{0\} \neq \tilde{U}(r) \subset L^{\mathrm{u}}$ and $\tilde{s}(r) \subset L^{S}$ for the local invariant manifolds $\tilde{U}(r)$ and $\tilde{S}(r)$ obtained from the application of Theorem II.1.1 to the restriction, $F \mid L_{r}^{*}$.

(4) DF is uniformly continuous on some neighborhood of $0 \in I$.

(5) $y_{-j} \in \tilde{U}(r), y_{j} \in \tilde{S}(r)$, and $F^{2 j}$ maps an open neighborhood $\Delta_{u}$ of $y_{-j}$ in $\tilde{U}(r)$ diffeomorphically onto its image $F^{2 j}\left(\Delta_{u}\right)$ which is a $C^{1}$-submanifold of $I$ with $T_{Y_{j}} F^{2 j}\left(\Delta_{u}\right) \oplus T_{Y_{j}} \tilde{S}(r)=L$.

The notation in (1) - (5) is as in Chapter II. 
We indicate how to verify these hypotheses for a restricted iterate of the map $P^{\prime}$ in suitable coordinates, provided the underlying nonlinearity $g$ is $c^{2}$. It is clear from the constructions in Chapters I, III, IV that $c^{2}$-smoothness can be achieved.

Then the semi-flow $x$ of eq. $(g)$ is $c^{2}$ on $(2, \infty) \times c$, and one can use the Implicit Function Theorem to show that all iterates $P^{n}$ and $\left(P^{\prime}\right)^{n}$, $2 \leqq n \in \underline{\mathbb{N}}$, are $c^{2}$.

1. In order to satisfy (4), one introduces neighborhoods of 0 in $L^{u}$ and in $I^{s}$ so that the restrictions of the maps $u$ and $s$ from section III.2 are $C^{2}$. $\left(\mathrm{P}^{2}\right.$ is $\mathrm{C}^{2}$, and close to $\phi^{*}$ local stable and unstable manifolds for $\mathrm{P}^{2}$ coincide with the local stable and unstable manifolds $\mathrm{S}$ and $U$ of $P$ from section III.2.)

As in section II.1, there is a $C^{2}$-diffeomorphism $T: D_{T} \rightarrow H$ from an open neighborhood $D_{T}$ of $\phi^{*}$ onto an open neighborhood $V_{T}$ of $0 \in H$, with

$$
\begin{aligned}
& T\left(\phi^{*}\right)=0, \quad D T\left(\phi^{*}\right)=i d, \\
& T\left(U \cap D_{T}\right)=L^{L} \cap V_{T}, T\left(S \cap D_{T}\right)=L^{S} \cap V_{T} .
\end{aligned}
$$

2. Choose $\nu \in \underline{N}$ with

$$
x_{n \nu} \in U \cap D_{T} \text { for } n \in-\underline{N}, x_{n v} \in S \cap D_{T} \text { for } n \in \underline{N} \text {. }
$$

The domain $D_{(2 v)}^{\prime}$ fof the iterate $\left(P^{i}\right)^{2 \nu}$ is an open neighborhood of the fixed point $\phi^{*}$ which contains alI " $\chi_{n}, n \in \underline{z}$. It follows that the points $\ldots x_{-3 v}, x_{-v}, x_{v}, x_{3 v}, \ldots \quad$, or better

$$
x_{n}^{*}:=x_{(2 n-1) \cup} \text { for } n \in \underline{z}
$$

form a homoclinic trajectory of the restriction

$$
\left(P^{\prime}\right)^{2 v} \mid D_{(2 v)}^{\prime} \cap D_{T} \text {. }
$$

We have $x_{n}^{*} \neq \phi^{*}$ for all $n \in \underline{z}$.

In order to get a map with range in $D_{T}$, set

$$
D^{*}:=\left[D^{\prime}(2 v) \cap D_{T}\right] \cap\left(\left(P^{\prime}\right)^{2 \nu}\right)^{-1}\left(D_{T}\right)
$$

and

$$
P^{*}:=\left(P^{\prime}\right)^{2 v} \mid D^{*}
$$

Clearly

$$
\underline{P}^{*}\left(D^{*}\right) \subset D_{T_{1}}, \phi^{*} \in D^{*}, x_{\mathrm{n}}^{*} \in D^{*} \text { for all } \mathrm{n} \in \underline{\underline{z}} .
$$

P* is $C^{2}$ since it is given by an iterate of $\left(P^{\prime}\right)^{2}$.

Corollary IV.4.4, Theorem II.1.1 (iv) and the definition of P' yield

(6) $\quad D\left(P^{*}\right)^{1-n}\left(X_{n}^{*}\right) v^{\prime} \notin T_{X_{1}^{*}} S$ for all integers $n \leqq 0,1>0$

if $\quad 0 \neq v^{\prime} \in \cdot T_{X_{n}^{*}} U^{U}$.

3. Set $V:=T(D *)$. The transformed map $F: V \rightarrow H$,

$F(\phi)=T\left(P^{*}\left(T^{-1}(\phi)\right)\right)$ for $\phi \in V$, is $C^{2}$ so that (4) is satisfied, and we have $F(0)=0, F(\dot{V}) \subset V_{T}$.

The points $\phi_{\mathrm{n}}:=\mathrm{T}\left(\chi_{\mathrm{n}}^{*}\right), \mathrm{n} \in \underline{\mathrm{Z}}$, form a homoclinic sequence of $F$, $\phi_{\mathrm{n}} \rightarrow 0$ as $|\mathrm{n}| \rightarrow \infty$ and $\phi_{\mathrm{n}} \neq 0$ for all $\mathrm{n} \in \underline{z}$.

Set $A:=D F(0)=D P\left(\phi^{*}\right)^{2 \nu}$. We see that 0 is a hyperbolic fixea point of $F$, and we obtain (1) with $L^{u}, L^{S},||^{*}$ from section III.2, and with some $q \in(0,1)$. Choose $c=c_{F}>0$ with

$$
q+c<1<q^{-1}-c
$$

and $r^{\prime}>0$ so small that

(2') for all $\phi \in I_{r^{\prime}}^{u *}+I_{r^{\prime}}^{s *} \in V$,

$\left|\mathrm{P}^{\mathrm{L}}[D F(\phi)-\mathrm{A}]\right|^{*}+\left|\mathrm{P}^{\mathrm{S}}[\mathrm{DF}(\phi)-\mathrm{A}]\right|^{*}+|\mathrm{DF}(\phi)-\mathrm{A}|^{*}<\mathrm{c}$.

4. In order to obtain (3) one shows first that there exists $r=r_{F}$ in $\left(0, r^{\prime}\right)$ with

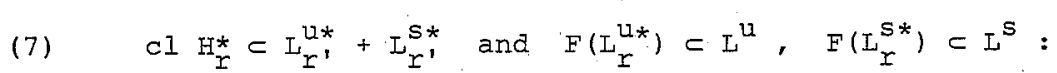

Theorem II.1.1 implies that there is a neighborhood $\tilde{D} \subset D_{T}$ of $\phi^{*}$ in $H$ such that for $\phi \in U \cap \tilde{D}$, the iterates $P(\phi), \ldots, P^{2 \nu}(\phi)$ are defined with

$$
\text { U } \ni P(\phi)=P^{\prime}(\phi), \ldots, U \ni P^{2 \nu}(\phi)=\left(P^{\prime}\right)^{2 \nu}(\phi),
$$

while for $\phi \in S \cap \tilde{D}$,

$$
S \ni P(\phi)=P^{\prime}(\phi), \ldots, S \ni P^{2 \nu}(\phi)=\left(P^{\prime}\right)^{2 \nu}(\phi) .
$$

Choose $x=r_{F} \in\left(0, r^{\prime}\right)$ so small that

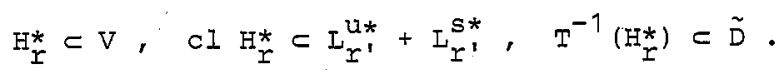

For $\phi \in I_{r}^{u *}$, use

$$
\begin{aligned}
& P^{*}\left(T^{-1}(\phi)\right)=P^{2 v}\left(T^{-1}(\phi)\right) \in U ; \\
& F(\phi)=T\left(P^{*}\left(T^{-1}(\phi)\right)\right) \in T\left(U \cap D_{T}\right) \subset L^{U} ;
\end{aligned}
$$

similarly for $\phi \in \mathrm{I}_{\Upsilon}^{\mathrm{S*}}$. 
5. The first inclusion in (7) and (2') yield (2).

6. Verification of (3). Consider the local stable and unstable manifolds $\tilde{S}(r)$ and $\tilde{U}(r)$ obtained from the application of Theorem II.1.1 to $F \mid H_{r}^{*}$.

Proof of $\tilde{U}(r) \subset L^{u}:$ Let $\psi \in \tilde{U}(r)$. There is a trajectory $\left(\psi_{n}\right)_{-\infty}^{0}$ of $F$ in $H_{r}^{*}$ with $\psi_{0}=\psi_{0}$. Recall Corollaxy II.1.5 and its proof - the properties (7) and (2') imply

$$
\left|p^{s} \psi\right|^{*}=\left|p^{s} F\left(\psi_{-1}\right)\right|^{*} \leqq(q+c)\left|p^{s} \psi_{-1}\right|^{*} \leqq \ldots \leqq(q+c)^{n} r^{\prime}
$$

for all $n \in \underline{N}$. Hence $p^{s_{\psi}}=0, \psi \in I^{u}$.

The proof of $\tilde{S}(r) \subset L^{S}$ is analogous, with $1<q^{-1}-c$.

$\{0\} \underset{f}{\subset} \tilde{U}(r)$ is clear since $\tilde{U}(r)$ and $\tilde{S}(r)$ are open subsets of $L^{u}$

and $L^{S}$, respectively.

7. Verification of the transversality condition (5). Choose $j=j_{F}$ in $\underline{N}$ with

$$
\phi_{-j} \in \tilde{U}(x) \subset I^{u}, \phi_{j} \in \tilde{S}(r) \subset I^{S} .
$$

observe that for all $\phi$ in the domain $V_{(2 j)} \in V$ of $F^{2 j}$,

$$
F^{2 j}(\phi)=T\left(\left(P^{*}\right)^{2 j}\left(T^{-1}(\phi)\right)\right) \text {. }
$$

we have

$$
\phi_{-j} \in V_{(2 j)}, \phi_{j}=F^{2 j}\left(\phi_{-j}\right) .
$$

Property (6) implies that $\mathrm{DF}^{2 j}\left(\phi_{-j}\right)$ maps the one-dimensional tangent space $T_{\phi} \tilde{U}(x)=L$ onto a one-dimensional space which is a complement of the closed subspace. $T_{\phi_{j}} \tilde{S}(x)=L^{S}$ in. $H$. It follows that $F^{2 j}$ maps an open neighborhood $\Delta_{u}$ of $\phi_{j}$ in $\tilde{U}(r)$, i.e., an open subset of $L^{u}$, diffeomorphically onto its image which is a one-dimensional $c^{2}$-submanifold of $H$, transversal to $\tilde{s}(r) \subset L^{S}$ at $\phi_{j}$.

\section{Mathematisches Institut}

Universität München

Theresienstr. 39

D 8000 München 2
[1] R. Abraham, J. Robbin: Transversal Mappings and Flows. Benjamin, New York 1967

[2] C.I. Cowan, z.j. Jelonek: Synchronized Systems with Time Delay in the Loop. Proc. Inst. Radio Engrs. 41, 388 - 397 (1957)

[3] J. Dieudonne : Foundations of Modern Analysis. Academic Press, New York 1960

[4] N. Dunford, J.T. Schwartz: Linear Operators I. Interscience, New York 1967

[5] T. Furumochi: Existence of Periodic Solutions of One-Dimensional Differential-Delay Equations. Tôhoku Math. J. 30, 13 - 35 (1978)

[6] W.H. Gottschalk, G.A. Hedlund: Topological Dynamics. Am. Math. Soc., Providence 1955

[7] J.K. Hale: Theory of Functional Differential Equations. Springer, New York 1977

[8] J.K. Hale, X.B. Lin: Symbolic Dynamics and Nonlinear Semiflows. Ann. Mat. Pura Appl. 144, 229 - 259 (1986)

[9] J.K. Hale, X.B. Iin: Examples of Transverse Homoclinic Orbits in Delay Equations. Nonlinear Analysis 10, 693 - 709 (1986)

[10] U. an der Heiden, H.O. Walther: Existence of Chaos in Control Systems with Delayed Feedback. J. Diff. Equ. 47, 273 - 295 (1983)

[11] U. an der Heiden, H.O. Walther: Chaos in Differential Delay Equations. In: The 9th Int. Conf: on Nonlinear Oscillations. Vol.2, 88 - 91. Kiev 1984

[12] M.C. Irwin: Smooth Dynamical Systems. Academic Press, London 1980

[13] J.I. Kaplan, J.A. Yorke: Ordinary Differential Equations which yield Periodic Solutions of Differential-Delay Equations. J. Math. Analysis Appl. 48, 317 - 324 (1974)

[14] T. Kato: Perturbation Theory. for Linear Operators. 2nd Ed., Springer, Berlin 1976

[15] T.Y. Li, J.A. Yorke: Period Three implies Chaos. Am. Math. Monthly 82,985 - 992 (1975)

[16] F.R. Marotto: Snap-Back Repellers imply Chaos in $\underline{R}^{n}$. J. Math. Analysis Appl. 63, $199-223$ (1978)

[17] J.K. Moser: Stable and Random Motions in Dynamical systems. Princeton Univ. Press, Princeton 1973

[18] A. Neugebauer: Invariante Mannigfaltigkeiten und Neigungslemmata für Abbildungen in Bànachräumen. Diploma thesis, Math. Inst. Univ. München, 1988 
[19] S.E. Newhouse: Lectures on Dynamical Systems. In: Dynamical Systems. CIME Lectures Bressanone 1980. Birkhäuser, Boston 1980

[20] R.D. Nussbaum: Uniqueness and Nonuniqueness for Periodic Solutions of $x^{\prime}(t)=-g(x(t-1))$. J. Diff. Equ. $34,25-54$ (1979)

[21] K.J. Palmer: Exponential Dichotomies and Transversal Homoclinic Points. J. Diff. Equ. 55, 225 - 256 (1984)

[22] K.J. Palmer: Exponential Dichotomies, the Shadowing Lemma and Transversal Homoclinic Points. In: Dynamics Reported. vol. $1,265-306$. Teubner/Wiley, Stuttgart 1988

[23] H. Peters: Globales Lösungsverhalten zeitverzögerter Differential-gleichungen am Beispiel von Modellfunktionen. Ph.D. thesis, Bremen 1980

[24] A.N. Sarkovski into itself. Ukrainian Mat. Z. 16, $61-.71$ (1964)

[25] L.P. Silnikov: On a Poincare'-Birkhoff Problem.

Math. U.S.S.R. Sbornik 3, 353 - 371 (1967)

[26] S. Smale: Diffeomorphisms with Many Periodic Points. In: Differential and Combinatorial Topology, 63 - 80. Princeton Univ. Press, Princeton 1965

[27] S. Smale: Differentiable Dynamical Systems. Bull. Am. Math. Soc. 73, $747-817$ (1967)

[28] H. Steinlein, H.O. Walther: Hyperbolic sets and Shadowing for Noninvertible Maps. In: Proc. Advanced Topics in the Theory of Dynamical Systems (Trento 1987). Academic Press, to appear

[29] H. Steinlein, H.O. Walther: Hyperbolic Sets, Transversal Homoclinic Trajectories and Symbolic Dynamics for $C^{1}$-Maps in Banach Spaces. Preprint 1988, submitted

[30] Y. Ueda: Self Excited Oscillations and their Bifurcations in Nonlinear Differential-Difference Equations. In: 24 th Midwest Symp. on Circuits ane Systems. Univ. of New Mexico, Albuquerque 1981

[31] H.O. Walther: Bifurcation from Periodic Solutions in Functional Differential Equations. Math. Z. 182, 269 - 289 (1983)

[32]. H.O. Walther: Homoclinic Solution and Chaos in $\dot{x}(t)=f(x(t-1))$. Nonlinear Analysis 5, 775 - 788 (1981)

[33] H.O. Walther: Bifurcation from a Heteroclinic Solution in Differential Delay Equations. Trans. Am. Math. Soc. 290, $213-233$ (1985)

[34] H.O. Walther: Bifurcation from a Saddie Connection in Functional Differential Equations: An Approach, with Inclination Lemmas. DISSERTATIONES MATH., to appear

[35] H.O. Walther: Homoclinic and Periodic Solutions of Scalar Differential Delay Equations. In: Proc. of the Semester on Dynamical Systems and Exgodic Theory. Banach Center Publications, Warsaw, to appear

[36] H.O. Walther: Inclination Lemaias with Dominated Convergence. J. Appl. Math. Phys. 32, $327-337$ (1987) 